\title{
An Intuitive Logic for Modeling Non-Local Hidden Variable Problems
}

Keith Fox ( $\square$ kmcauliffefox@gmail.com )

None https://orcid.org/0000-0002-2994-2080

Tyler White

Northern Virginia Community College - Loudoun Campus

\section{Research Article}

Keywords: Formal Language, Structure, Substructure, Semantics, Hidden Variable, Superposition, Entanglement, Modeling

Posted Date: July 26th, 2022

DOI: https://doi.org/10.21203/rs.3.rs-403177/v3

License: (c) (1) This work is licensed under a Creative Commons Attribution 4.0 International License. Read Full License 


\title{
An Intuitive Logic for Modeling Non-Local Hidden Variable Problems
}

\author{
Keith Fox ${ }^{1}$, Tyler White ${ }^{2}$
}

\begin{abstract}
We present a notion of an un-measurable state, which we call an Anti-state and derive a simple and intuitive logic for Anti-states. Anti-states are then used to generate structures, which we call Anti-Structures. Theorems 3.25 and 4.2 yield that Anti-Structures are able to model the context changes (and inconsistencies of measured values), occurring in quantum mechanics, where Kochen and Specker in [5] showed these were un-modellable with local hidden variables. In our Examples 3.28, 4.3, and 4.7, we show that, respectively, the orthohelium experiment (outlined in Kochen, Specker [5]), wave functions, and entanglement all have Anti-Structure models. Our results give a good contrast to the results of Kochen and Specker [5], and establish a limitation on self reference for partial Boolean algebras. This limitation is not held by Anti-Structures in general, and also does not seem to be a limitation that should be put on our physics. The theorems and examples of this paper lead to a series of questions regarding the expressiveness and a measure of contrivance for models of quantum mechanics.
\end{abstract}

Keywords: Formal Language, Structure, Substructure, Semantics, Hidden Variable, Superposition, Entanglement, Modeling.

\section{Prologue}

After much communication regarding the length of the paper, it is clear that some assurance regarding its length should be given. To give an assurance that the length is worth the exercise on the readers part, we point out that the nature of the paper is to both introduce an intuitive notion as well as give formal and rigorous justification for it. Those fortunate enough to have studied the foundations of mathematics will know that familiar simple every day mathematical concepts can, when formalized, seem dauntingly complex. Indeed even an elegant three page argument delivering significant results in physics, when unpacked to its mathematical foundations and completely justified, should run hundreds of pages. We ask that for the readers benefit they view the length of this paper as being due to its performing the tasks of, in exposition presenting

\footnotetext{
${ }^{1}$ Unaffiliated: kmcauliffefox@gmail.com

${ }^{2}$ Northern Virginia Community College: tmwhite@nvcc.edu
} 
an unfamiliar intuitive idea, while mathematically giving rigor and insight. It should also be made clear that to either read the mathematics, ignoring the exposition, or to read the exposition, ignoring the mathematics, would leave the reader wholly uninformed about of the results of this paper and would produce only a waist of time.

\section{Introduction}

We begin with the simplest summary of the intuition underlying this paper. This being that context changes due to measurement can be modeled by removing points from standard models. These removed points are then wholly lost to our discourse and as such, all meaning and associated meaning of their measurement has been taken away. These removed points will be referred to as Anti-states, and the models after the points have been removed will be AntiStructures. This paper is then dedicated to rigorously establishing the notions of Anti-state and Anti-Structure, as well as demonstrating that they give a simple logical mechanism for producing context change. The end of the paper explores conditions that would give rise to Anti-states and Anti-Structures and contains questions which we believe establish a framework for further research. The authors strongly feel that we are presenting a purely mathematical construct and not giving an explanation of real world processes, and the reader will find that our exposition strongly maintains this position throughout. The fact that we are giving purely mathematical mechanisms for context change should not detract from the potential contributions of these ideas to real world explanations. Indeed as stated in the abstract, we believe that Anti-states and Anti-Structures in their minimalism present a means of gauging the complexity of the process of context change presented by any existing and future models of QM. The outline of this paper is as follows:

- In Section 2 we give a general summary of Kochen and Specker's paper [5].

- In Section 3 we establish the notion of an Anti-Structure $\models_{\mathfrak{A}}^{A}$ which defines our Anti-states. We give initial examples of Anti-Structure models and we discuss how these models capture the context changes caused by measurement in quantum mechanics. The connection between the AntiStructure models and the context changes due to measurement occurring in quantum mechanics is given by our Theorem 3.10 which establishes the necessary and sufficient conditions on a mathematical model for it to correctly assign truth to formulas representing context change. We then with Example 3.11 demonstrate the effect of context change due to measurement in an Anti-Structure. To fully understand and extend our ability to model with Anti-Structures we define a system 3.14 which gives a set of formulas that are seen in Lemma 3.17 to be unique to each Anti-Structure and in Theorem 3.18 are shown to always give rise to an Anti-Structure. We use the notion of a system to extend our modeling 
with Anti-Structures to what we call simple contextual models (Definition 3.20). A contextual model (see Definition 3.20) is anything that gives the truth of formulas by reading the formula and looking up an ascribed truth value for the formula. We give as a subclass of contextual models, simple contextual models 3.20, where then our Theorem 3.22 allows us to generate Anti-Structure models which agree with the truth values of the simple contextual model. Example 3.28 demonstrates that the set of measurements for the orthohelium example of [5] can be captured by a simple contextual model having a corresponding Anti-Structure model. In Remark 3.28 we sum up the significance of giving an Anti-Structure model for Kochen and Speckers orthohelium example by noting that our model demonstrates the consistency of the statements:

i. No local hidden variable theory can account for the inconsistencies in measured values.

ii. The inconsistencies in measured values occurring in the experiment can be obtained from exactly what can be measured in the given experiment, when an Anti-Structure model is used.

We proceed in Section 3 by motivating our use of the operator Slowed (see Definition 3.36) to give Anti-Structures as models for a more general class of contextual models, which we call rational contextual models (see Definition 3.35 and the construction of example 3.33). Example 3.33 demonstration a notion of entanglement for simple contextual models and motivates both the rational contextual models and the Slowed operator. We complete this section with Theorem 3.37 and its Corollary 3.39 which allow us to attain an Anti-Structure model for every rational contextual model. The rational contextual models in addition to being able to model entanglement, are necessary in our construction of probabilistic Anti-Structure models (in section 4) that model wave functions and their measurement.

- In Section 4 we establish a notion of a probabilistic contextual model (definition 4.1) to allow us to model the statistical nature of quantum mechanics. We give our Second Model Completeness theorem 4.2, which shows that our probabilistic contextual Models can be given with an AntiStructure. Similar to our operator Slowed we give an operator Spaced (definition 3.37) where this operator facilitates a simple proof of Theorem 4.2. We apply our Theorem 4.2 to attain a model of all wave functions (example 4.3), and an example of probabilistic models of both the double slit experiment 4.6, as well as an example of entanglement and the double slit experiment 4.7 .

- Section 5 has subsections for: important pathological Examples 5.1, Comments 5.2, and further Questions 5.3. Our subsection for pathological examples gives Examples 5.1 and 5.2 which are pathological in the sense that: 
- Example 5.1 demonstrates that it is possible to have Anti-Structures which can represent their own truth, where these Anti-Structures support the theory of the natural numbers to the degree that any standard structure, supporting the same degree of the theory of the natural numbers, would not be able to represent their own truth in accordance with Gödel's incompleteness theorem see [1].

- Example 5.2 demonstrates how an Anti-Structure can be used to give finite models of sets of statements $S$ where every standard structure model for $S$ must be infinite.

In the exposition of this section we discuss some interesting similarities the examples have to observed phenomena, where in the comments section we look at the consequences of Example 5.1 to a notion of expressiveness (Definition 5.5) which partial Boolean algebras fail to attain (see Comment 1 in Subsection 5.2). In the comments section we make note of the ability to take the results of this paper in the context of more general methods of assigning truth (see Definition 5.3). Our discussion of the results of this paper to more general methods of assigning truth leads to an interesting connection between the necessity of the Plank length and a notion of existential crisis (see Definition 5.6). In the further questions section we turn much of the discussion in the comments section into formal questions. Directly motivated by the examples of this paper and discussed in the comments section (see Comment 2 in Subsection 5.2) is the overall theme of our questions, which is that of :

- Addressing the level to which a physicists model of QM (or our universe) must know itself (in the Gódel sense).

- Determining a standard for how natural versus how constructed any given model of QM is. This can be seen as establishing a notion of how much of an appeal to external authority is required for a given model to exist.

\section{Local versus Contextual Hidden variables}

The 1967 paper by Kochen and Specker [5] showed that the mathematics describing Quantum Mechanics (QM) gives partial Boolean algebras. The algebra was partial in the sense that not every molecular sentence has meaning, even though every atomic sentence always has meaning. They showed that there are partial Boolean algebras arising from QM that cannot be embedded into a Boolean algebra. Kochen and Specker argue that if a scientist "believes in hidden variables, he should be able to predict (in theory) the measured value of every quantum mechanical observable." [5]. They turn an experiment on orthohelium into a partial Boolean algebra which is not embeddable in a Boolean algebra. This leads to the contradiction that the angular momentum, in a certain direction of measurement of the orthohelium, must have two different values simultaneously. Thwarting any scientists attempt to predict the outcome. This 
establishes a local hidden variable cannot be used to model Quantum Mechanics. However, if we assume that not all atomic sentences have a truth value, arising from what we call an Anti-state, our Anti-state can be assigned to satisfy the intent of a local hidden variable and give a mathematical model discerning outcomes using fundamental and general logical principles.

We argue that this model has several advantages over partial Boolean algebras. The limitation we are most concerned with (discussed further in the sequel) is that a partial Boolean algebra is not expressive, which yields that it cannot check its own truth in a meaningful way (See Example 5.1 and Section $5.2)$.

\section{Anti-states, Anti-Structures and Basic Models of Contextuality}

Partial Boolean algebras assign additional requirements on our logical connectives, in the sense that, in a partial Boolean algebra formulas $\phi$ and $\psi$ can have Boolean values but $\phi \wedge \psi$ does not. A use of Anti-states and Anti-Structures allows for the attainment of the results of the partial Boolean algebras, while removing the aforementioned extra duty of the logical connectives. The logic for Anti-Structures has a motivation in understanding the standard interpretation of statements of the form: In $\models_{\mathfrak{A}}$, one sees (or doesn't see) such and such. To understand the nature of this statement, we note that Gödel's completeness theorem gives countable models for all countable sets of consistent first order formulas. A consequence of this being that because there exists a countable and consistent set of first order sentence which give the first order theory of the real line [1], there exists a countable model of the real line. And therefore we have a structure $\mathfrak{A}$ where $|\mathfrak{A}|$ is countable, and $\models_{\mathfrak{A}}$ gives the first order theory of the real line. Consequently, we would say in $\models_{\mathfrak{A}}$ one cannot see that the lines cardinality is not equal to the continuum. Perhaps a better example of this is seen in forcing, where one has countable models $\mathfrak{A}_{i}$ of ZFC (or significant parts of ZFC, see Kunnen's book on set theory and independence proofs [3]) where given the continuum is $\mathfrak{c}$ and the $i$ 'th uncountable cardinal is $\omega_{i}$ for $i \in \mathbb{N}$, we have $\models_{\mathfrak{A}_{1}}\left(\mathfrak{c}=\omega_{1}\right)$ and $\models_{\mathfrak{A}_{2}}\left(\mathfrak{c}=\omega_{2}\right)$. We would then say that $\models_{\mathfrak{A}_{1}}$ sees the continuum as the first uncountable cardinal, and $\models_{\mathfrak{A}_{2}}$ sees the continuum as the second uncountable cardinal, (see [3]). It should be noted that in both cases there are countably infinite sets $S_{1}$ and $S_{2}$ where in $\models_{\mathfrak{A}_{i}}, S_{i}$ is seen to be $\mathfrak{c}$, so that $\models_{\mathfrak{A}_{i}}$ does not see the bijection from $S_{i}$ to the natural numbers that we see in the meta theory (again we refer the reader to [3]). The underlying interpretation of the statement can be given in the following: Given we have a point set $X$, a set of functions $F \subseteq\left\{f \mid f: X^{n} \rightarrow X\right.$ where $\left.n \in \mathbb{N} \cup\{0\}\right\}$ (note $\mathbb{N}$ denotes the natural numbers here, and when $n=0, f$ denotes a constant), a set of subsets $P \subseteq\{u \mid u \subseteq X\}$, and given a person, call him Peter, where Peter wants to rationally study $\langle X, F, P\rangle$, then Peter is assumed to have the following:

- A formal language $L$ which allows Peter to give formulas in first order logic. 
- A tool $\models_{\mathfrak{A}}$ which when Peter takes a formula $\phi$ of the language $L$ and a variable assignment $[v]$ and gives it to the tool, as such $\models_{\mathfrak{A}} \phi[v]$, then the tool returns true or false, where the true means that $\phi$ under $[v]$ is a true statement about $\langle X, F, P\rangle$, and the false would mean that it is not true.

In this context when we talk about what $\models_{\mathfrak{A}}$ sees, we are referring to what this tool tells Peter about $\langle X, F, P\rangle$. The mathematicians are not using said tool, but rather studying Peters use of the tool, and Peter is only studying $\langle X, F, P\rangle$. The reason we are belaboring the explanation of how we view the the perspective the mathematician has on $\models_{\mathfrak{A}}$ is to make it clear that in all instances of $\models_{\mathfrak{A}}$, the object it is returning information about is $\langle X, F, P\rangle$ and only in the meta theory do we study its relationship to the formulas of $L$. We study Peter(Not as a person but as a process and indeed Peter can be replaced with an algorithm plugging formulas into $\left.\models_{\mathfrak{A}}\right)$, how $\models_{\mathfrak{A}}$ works, and $\langle X, F, P\rangle$, while Peter only studies $\langle X, F, P\rangle$. To these ends traditionally when we say that $\models_{\mathfrak{A}}$ gives a model of something, then that something is of the form $\langle X, F, P\rangle$ and our logical connectives $\vee, \wedge, \rightarrow, \neg$ as well as our quantification $\forall, \exists$ and any formulas $\phi$ of our language are not a part of $\langle X, F, P\rangle$ and have no bearing on its nature. And as such the formulas are for our understanding or Peters understanding of $\langle X, F, P\rangle$ where $\langle X, F, P\rangle$ makes no use of formulas or their logical connectives. To note the contrast of the interpretation of the logical connectives with regard to models in quantum theory, to the classical interpretation we have given, we take from Kochens paper [6], the following excerpt which is from Birkhoff and von Neumann in [8], but we have it as it appears in [6]: "What experimental meaning can one attach to the meet and join of two experimental propositions?" Where then Kochen gives: "That question has never been adequately answered." It is clear from the preceding that the logical operators are in a different role in modeling quantum mechanics ( and in partial Boolean algebras), as apposed to their role in standard mathematical models. To motivate an understanding of this, we give an outline of the double slit experiment as described by Feynman in [2]. In his example Feynman has a source which is emitting particles at a barrier wall, where there are two holes or slits which we will call $A$ and $B$. The other side of the barrier wall (relative to the emitter) has a movable detector $D$ on its own wall (which we will call the back wall), which is parallel to the barrier wall. When the experiment is conducted making measurements at $A$ and at $B$, the detector is moved over time to all positions on the back wall and the data gives the following:

- A particle goes from source to back wall only after being measured at either $A$ or $B$. (this is not explicitly stated in [2])

- When particles are measured at $A$ and $B$ then they predominantly are measured at the back wall directly behind $A$ and $B$.

When the experiment is conducted making no measurement at $A$ and $B$, the detector at the back wall predominantly measures particles hitting in an interference pattern all along the back wall with the likely hood of being measured 
directly behind $A$ and $B$ being diminished. From these two experiments we get the following statements

1. All particles that hit the back barrier when measured at $A$ or $B$ must pass through $A$ or $B$ and when a particle passes through $A$ or $B$, then almost always it is measured at the back wall directly behind $A$ or $B$.

2. Almost all particles that hit the back wall will end up being detected in an interference pattern and relatively few of the measured particles will be measured directly behind $A$ and $B$.

The contradiction here is clear. However, we give the above statements a more symbolic look to see the exact form of this contradiction. To add some clarity and simplify our setup we will alter statement (1) above by replacing almost all with all, and we will alter statement (2) by replacing relatively few with none. We will have $A B(x), B W(x)$ and $D B(x)$ be unary predicates where $A B(x)$ will represent the statements that $x$ is measured at $A$ or $B, B W(x)$ will represent that $x$ is measured at the back wall and $D B(x)$ will represent that $x$ is measured directly behind $A$ and $B$. Given our point set is particles from the emitter we then have from 1 and 2 the following statements:

$$
\begin{aligned}
& \text { I. } \forall x[(B W(x) \rightarrow A B(x)) \wedge(A B(x) \rightarrow D B(x))] \\
& \text { II. } \exists x[B W(x) \wedge \neg D B(x)]
\end{aligned}
$$

It then follows that if both I and II are true, then the logically valid formula $(\forall x((B W(x) \rightarrow A B(x)) \wedge(A B(x) \rightarrow D B(x)))) \rightarrow \forall x(B W(x) \rightarrow D B(x))$ must be false (Note logically valid formulas are always true in standard logic). We note that the falsity of the preceding logically valid formula is exactly the type of contradiction which is referenced by Kochen and Specker in [5] in their Introduction when they assert: It then follows from our results that there is a formula $\psi\left(x_{1}, \ldots, x_{n}\right)$ which is a classic tautology but is false for some meaningful substitution of quantum mechanical propositions. We note that the above logically valid formula has $((P \rightarrow Q) \wedge(Q \rightarrow R)) \rightarrow(P \rightarrow R)$ as a tautological counterpart in propositional logic. Taking the preceding logically valid formula as $\phi$, it is clear that in any traditional structure $\mathfrak{A}$ of a language that contains $\phi$ as a formula, we have $\models_{\mathfrak{A}} \phi$. The partial Boolean algebra of [5] allows for a semantics where formulas such as $\phi$ can be false as is implied by the preceding except from [5]. Shortly we will define $\models_{\mathfrak{A}}^{A}$ and in Example 3.5 we will have a $\models_{\mathfrak{A}}^{A}$ where $\models_{\mathfrak{A}}^{A} \phi$ is false. Again the significance here will be in how naturally $\models_{\mathfrak{A}}^{A}$ follows from $\models_{\mathfrak{A}}$. We refer the reader to [5] or [7] for how a partial boolean algebra gives a semantics where formulas like $\phi$ can be false.

We define an Anti-structure $\models_{\mathfrak{A}}^{A}$ from a structure $\models_{\mathfrak{A}}$ as follows (Note that the following is a matter of rigor and we omit no details): 
Definition 3.1. Given a formula $\psi$, we will call a variable $x$ a terminal variable of $\psi$ if and only if there is an occurrence of $x$ in $\psi$, other than $\forall x$ or $\exists x$, where $x$ is not the argument for a function symbol.

Definition 3.2. Given a formula $\phi$ we will have $t \in \operatorname{ter}(\phi)$ if and only if there is a formula $\psi$ having a terminal variable $x$, where the formula $\phi$ can be attained by substituting $t$ for $x$ in $\psi$. We will call $t \in \operatorname{ter}(\phi)$ a terminal term of $\phi$. Note $\operatorname{ter}(\phi)$ may include constant symbols and variables, as well as arbitrary terms.

Definition 3.3. Given $\mathfrak{A}$ is a structure for a first order language $\mathfrak{L}$ and $A \subseteq|\mathfrak{A}|$ (so that $|\mathfrak{A}|-A$ includes the set of Anti-states), we now define an Anti-Structure $\models_{\mathfrak{A}}^{A}$ in two parts (see[1] for the definition of a structure $\mathfrak{A}$ ). Please note that our definition of Anti-Structure can be viewed as simple modifications to the definition of $\mathfrak{A}$ given in [1]. And as such is no longer than need be. Also note that $\mathfrak{A}$ is the rigorous foundation for all models of our mathematics and science. Indeed if take any paper of modern physics giving a model, and pulled it back to its rigorous foundations, then the paper would require a structure $\mathfrak{A}$ to be absolutely complete and rigorous.

I. We will have for all variable assignment $s: \operatorname{Var} \rightarrow A$ and all quantifier free formula $\psi$ that $\models_{\mathfrak{A}}^{A} \psi[s]$ has the same truth value as $\models_{\mathfrak{A}} \psi[s]$ iff we have both:

1. For all constants $c$ occurring in $\psi$, it is the case that $c^{\mathfrak{A}} \in A$. Note that we are not necessarily referring to constants in $\operatorname{ter}(\psi)$ here.

2. For all $t \in \operatorname{ter}(\psi)$, we have $t[s] \in A$.

And $\models_{\mathfrak{A}}^{A} \psi[s]$ will have no defined truth value otherwise.

II For all formulas $\phi$ having no free variables we define $\models_{\mathfrak{A}}^{A} \phi$ is (true/false) recursively as follows:

From part (I) of this definition we have established the meaning of $\models_{\mathfrak{A}}^{A} \psi[s]$ for all quantifier free $\psi$ and variable assignments $s: \operatorname{Var} \rightarrow$ $A$.

* In what follows we establish the meaning of $\models_{\mathfrak{A}}^{A} \forall x \psi[s]$ and $\models_{\mathfrak{A}}^{A} \exists x \psi[s]$ recursively for all variable assignments $s: \operatorname{Var} \rightarrow A$ and all formulas $\psi$ with an unquantified variable $x$. Since each quantifier free formula has only a finite number of variables it will be the case that for any quantifier free formula $\psi$ and any variable assignment $s: \operatorname{Var} \rightarrow A$ there is a finite number of steps that will allow us to give meaning to $\models_{\mathfrak{A}}^{A} \phi[s]$ is (true or false) when $\phi$ is obtained by quantifying all variables in $\psi$.

Given $x$ is free in $\psi$ and we have an established truth value for

$$
\models_{\mathfrak{A}}^{A} \psi[s]
$$

, then we will have: 
$1 \models{ }_{\mathfrak{A}}^{A} \forall x \psi[s]$ is true if and only if for every variable assignment $s^{\prime}: \operatorname{Var} \rightarrow A$ where for all $y \neq x$ we have $s^{\prime}(y)=s(y)$ it holds that if $\models_{\mathfrak{A}}^{A} \psi\left[s^{\prime}\right]$ has a truth value, then $\models_{\mathfrak{A}}^{A} \psi\left[s^{\prime}\right]$ is true. Note that to determine the truth value for $\models_{\mathfrak{A}}^{A} \psi\left[s^{\prime}\right]$ we would have to apply our recursive definition.

$2=_{\mathfrak{A}}^{A} \exists x \psi[s]$ is true if and only if there exists a variable assignment $s^{\prime}: \operatorname{Var} \rightarrow A$ where for all $y \neq x$ we have $s^{\prime}(y)=s(y)$ and $\models_{\mathfrak{A}}^{A} \psi\left[s^{\prime}\right]$ is true.

3. $\models_{\mathfrak{A}}^{A} \forall x \psi[s]$ is false if and only if $\models_{\mathfrak{A}}^{A} \exists x \neg \psi[s]$ is true.

4. $\models_{\mathfrak{A}}^{A} \exists x \psi[s]$ is false if and only if $\models_{\mathfrak{A}}^{A} \forall x \neg \psi[s]$ is true

Remark 3.4. Noting that $|\mathfrak{A}|$ denotes the point set for the structure $\mathfrak{A}$, we have the following. When $A \subset|\mathfrak{A}|$ gives a substructure the above definition of $\models_{\mathfrak{A}}^{A} \phi$ agrees with the definition of $\models_{\mathfrak{A}}$ and consequently, when $A$ gives a substructure we have $\models_{\mathfrak{A}}^{A} \phi[s] \Leftrightarrow \models_{\mathfrak{A}} \phi[s]$. When $A$ does not give a substructure, then we will see that $\models_{\mathfrak{A}}^{A}$ can be very pathological when it comes to sentences having quantifiers (see Example 3.5). While for all quantifier free $\phi$ and all variable assignments $v$ if $\operatorname{ter}(\phi)^{\mathfrak{A}}[v] \subseteq A$, then $\models_{\mathfrak{A}}^{A} \phi[v]$ is equal to $\models_{\mathfrak{A}} \phi[v]$ and consequently we also have

$\left\{\phi: \phi\right.$ is a quantifier free sentence and $\left.\models_{\mathfrak{A}}^{A} \phi\right\}=$

$\left\{\phi: \phi\right.$ is a quantifier free sentence where $\operatorname{ter}(\phi)^{\mathfrak{A}} \subseteq A$ and $\left.\models_{\mathfrak{A}} \phi\right\}$ which

gives that $\models_{\mathfrak{A}}^{A}$ is not entirely pathological. We also note that the preceding also gives the base case for a straightforward proof by induction that for all sentences $\psi$ we will have $\models_{\mathfrak{A}}^{A} \psi$ is exactly one of true, false or undefined.

Before we proceed with our initial examples we need to analyze the semantics of $\models_{\mathfrak{A}}^{A}$ with regard to the notion of what it sees, or to pull from our earlier analogy, what kind of tool is $\models_{\mathfrak{A}}^{A}$ to Peter. Our fundamental assumption on $\models_{\mathfrak{A}}^{A}$ is that Peter only sees what is true and false and has no information or knowledge directly given to him from $\models_{\mathfrak{A}}^{A}$ where $\models_{\mathfrak{A}}^{A}$ is undefined.

We say that Peter gets no such information directly from $\models_{\mathfrak{A}}^{A}$ because we do not wish to put any constraints on whether Peter is capable of having $\models_{\mathfrak{A}}^{A} \psi$ where $\psi$ might encode a statement that $\models_{\mathfrak{A}}^{A}$ is sometimes undefined. Note in Section 5 we look at Gödel's incompleteness and what it says about the class of Anti-Structures and their expressibility. We note that Peter's not being able to see when $\models_{\mathfrak{A}}^{A}$ is undefined is similar to when a countable $|\mathfrak{A}|$ can be given where $\models_{\mathfrak{A}}$ is a model of ZFC, so that we would then say $\models_{\mathfrak{A}}$ does not know it is countable (for reference on countable models of ZFC, see [3]). With regard to the logical operators, to Peter they are in no way different than they are when using $\models_{\mathfrak{A}}$. Indeed, the way we have set up $\models_{\mathfrak{A}}^{A}$ it is not the logical connectives of a quantifier free formula $\phi$ that determine whether $\models_{\mathfrak{A}}^{A} \phi[v]$ has a truth value under a given variable assignment $v$, but rather whether the value of the terminal terms of $\phi$ under $v$, are or are not in $A$. Here the terminal terms are being considered the subject of $\phi$ and if those terms are not something we have access to(in an Anti-state), then we should not be able to have them as the subject of a statement $\phi$ having truth value. Note $\phi$ is interpreted as a 
statement asserting qualities of measured values, that are being represented by the terminal terms.

So Peter is using standard logic to formulate statements in which the subject of the statements can be directly observed. It should be noted that we are allowing for a terminal term (subject of a statement) to be arrived at by processes that may not be observable (may be in an Anti-state). Additionally this means that with regard to a term having undefined value, functional composition is not associative. To determine truth values for formulas with quantification, we are treating the set of all of the quantifier free formulas $\phi$ under variable assignment, which have a truth value in $\models_{\mathfrak{A}}^{A}$, as the data set of what can be observed, and then from this set and the assumption that we know no more or less than what is given by the set we deduce $\models_{\mathfrak{A}}^{A} \psi[s]$ for a formula $\psi$ having quantification. We will see later that the aforementioned data set (later called a system) will be consistent, and yet can be incomplete relative to what the structure $\models_{\mathfrak{A}}$ would give, and when incomplete our method for deducing the truth of sentences can yield that logically valid formulas are false (as mentioned above).

The next few examples will highlight this semantic choice for $\models_{\mathfrak{A}}^{A}$.

Example 3.5. Letting $|\mathfrak{A}|=\{a, b, c\}$ and $\mathfrak{L}$ be the first order language consisting of a constant symbol $s$ and a unary function symbol $f$. If we take $s^{\mathfrak{A}}=a$ and $f^{\mathfrak{A}}(x)=F$ where $F(a)=b, F(b)=c$ and $F(c)=a$, then we have $\mathfrak{A}$ gives a well defined structure in the point set $\{a, b, c\}$. Taking $A=\{a, c\}$ we now have that $b$ is our only Anti-state and we note the following about $\models_{\mathfrak{A}}^{A}$ :

1. $\models_{\mathfrak{A}}^{A}[(x=s) \vee(x=f f(s))][v]$ is true for all variable assignments $v$ with range a subset of $A$ and therefore we have $\models_{\mathfrak{A}}^{A} \forall x[(x=s) \vee(x=f f(s))]$ is true.

2. We now look at $\models_{\mathfrak{A}}^{A}[f(x)=y][v]$, letting $v$ range over all variable assignments with range a subset of $A$. In this case we see that $\models_{\mathfrak{A}}^{A}[f(x)=y][v]$ returns a truth value only when $v(x)=c$, and is true only when $v(x)=c$ and $v(y)=s^{\mathfrak{A}}=a$ and therefore we have $\models_{\mathfrak{A}}^{A} \forall x \exists ! y[f(x)=y]$.

3. Looking at $\models_{\mathfrak{A}}^{A}[f(x)=f f(s)][v]$, while letting $v$ range over all variable assignments with range a subset of $A$,

we have that $\models_{\mathfrak{A}}^{A}[f(x)=f f(s)][v]$ has a truth value only when $v(x)=c$ and in this case it returns a false, and therefore

$\models_{\mathfrak{A}}^{A} \exists x[f(x)=f f(s)]$ is false and therefore $={ }_{\mathfrak{A}}^{A} \forall x[f(x) \neq f f(s)]$ is true.

4. For $\models_{\mathfrak{A}}^{A}[f(s)=y][v]$, letting $v$ range over all variable assignments with range a subset of $A$,

we have that $\models_{\mathfrak{A}}^{A}[f(s)=y][v]$ never has meaning and consequently no generalizations can be inferred for it.

5. For $\models_{\mathfrak{A}}^{A}[f(x)=s][v]$, letting $v$ range over all variable assignments with range a subset of $A$, 
we have that $\models_{\mathfrak{A}}^{A}[f(x)=s][v]$ only has a truth value when $v(x)=c$ and in this case it is true and therefore we have $\models_{\mathfrak{A}}^{A} \forall x[f(x)=s]$ is true.

The pathological nature of the above (specifically the fact that the conclusions of 2,3 and 4 do not give a logically consistent set) might seem at first to be a sign of irreconcilable problems with the approach. However, the redemption of the Anti-Structure is in the fact that the above inconsistencies were arrived at through a rational algorithmic process and that these contradictions have a definable cause and an underlying structure governing them. There is zero mystery as to the mechanism behind the above statements, and more importantly this mechanism has been given as a well defined mathematical object where the above set of statements were not painstakingly constructed into said mechanism to give such pathological results, but rather were the natural consequence of simply removing a point from $|\mathfrak{A}|$. We remind the reader that the problem with modeling quantum mechanics is that no intuitive and natural mechanism seems to be governing the kinds of contradictory statements that arise in the quantum mechanics (we refer the reader to the outline of the double slit experiment given in the preceding).

\subsection{Conditions for Anti-Structures}

Proposition 3.6. For a structure $\mathfrak{A}$ for a language having $=$, if $A \subset|\mathfrak{A}|$, then $A$ is not a point set for a substructure of $\mathfrak{A}$ iff there exists a quantifier free formula $\psi$ and a variable assignment $s:$ Var $\rightarrow A$ where neither $\models_{\mathfrak{A}}^{A} \psi[s]$ or $\models{ }_{\mathfrak{A}}^{A} \neg \psi[s]$ are true.

Proof. $(\Rightarrow)$. If $A$ is not a point set for a substructure then either there exists a constant symbol $c$ where $c^{\mathfrak{A}} \notin A$ or there exists an n-ary function symbol $f$ and an $s: \operatorname{Var} \rightarrow A$ where $f\left(x_{1}, \ldots, x_{n}\right)[s] \notin A$. If there is a constant symbol $c$ where $c^{\mathfrak{A}} \notin A$, then we have neither $\models_{\mathfrak{A}}^{A}(c=c)$ or $\models_{\mathfrak{A}}^{A} \neg(c=c)$ is true. If there exists an n-ary function symbol $f$ and an $s: \operatorname{Var} \rightarrow A$ where $f\left(x_{1}, \ldots, x_{n}\right)[s] \notin A$, then neither $\models_{\mathfrak{A}}^{A}\left(f\left(x_{1}, \ldots, x_{n}\right)=f\left(x_{1}, \ldots, x_{n}\right)\right)[s]$ nor $\models \underset{\mathfrak{A}}{A} \neg\left(f\left(x_{1}, \ldots, x_{n}\right)=f\left(x_{1}, \ldots, x_{n}\right)\right)[s]$ is true.

$(\Leftarrow)$. If $A$ is the point set for a substructure of $\mathfrak{A}$, then we have $\models_{\mathfrak{A}}^{A}$ is $\models_{A}$ and consequently for all formulas $\psi$ exactly one of $\models_{A} \psi[s]$ or $\models_{A} \neg \psi[s]$ is true.

Corollary 3.7. The following are equivalent:

- $A \subseteq|\mathfrak{A}|$ is not a point set for a substructure.

- There is a constant $c$ or an $n$-ary function $f$ where either $c^{\mathfrak{A}} \notin A$ or there are $a_{1}, \ldots, a_{n} \in A$ where $f^{\mathfrak{A}}\left(a_{1}, \ldots, a_{n}\right) \notin A$.

- There exists a quantifier free formula $\psi$ and a variable assignment $s:$ Var $\rightarrow A$ where $\models_{\mathfrak{A}}^{A} \psi[s]$ has no truth value.

- There exists a quantifier free formula $\psi$ and a variable assignment $s:$ Var $\rightarrow A$ where neither $\models_{\mathfrak{A}}^{A} \psi[s]$ or $\models_{\mathfrak{A}}^{A} \neg \psi[s]$ are true. 
3.2. Modeling Contextuality In The Double Slit Experiment; The Observer Effect For Anti-Structures.

By Contextuality in the double slit experiment we are referring to the contradictory statements that we derived from the Feynman description of the double slit experiment, which we gave in the preceding. By the observer effect we mean the general form of the contradiction which was derived from Feynman's description of the double slit experiment and more generally statements about what is measured in a quantum system which lead to the failure of a tautology, see [5] or our reference to [5] given in the description of the double slit experiment in the preceding. In this section we are specifically giving what we call the observer effect in an Anti-Structure, while in Section 4 we will give a more rigorous connection of the observer effect in an Anti-Structure to the use of Anti-Structures in giving models of quantum mechanics and specifically the examples given in Kochen and Speckers paper on hidden variables [5].

Definition 3.8. Given $\mathfrak{A}$ is a structure for a first order language $\mathfrak{L}$, we will have that $A \subseteq|\mathfrak{A}|$ has an Anti-state relative to $\mathfrak{A}$ ( or $\models_{\mathfrak{A}}^{A}$ has an Anti-state) if and only if there exists a term $t\left(x_{1}, \ldots, x_{n}\right)$ of $\mathfrak{L}$ and a variable assignment $s$ with range in $A$ where $t^{\mathfrak{A}}\left(s\left(x_{1}\right), \ldots, s\left(x_{n}\right)\right) \notin A$ where then $t^{\mathfrak{A}}\left(s\left(x_{1}\right), \ldots, s\left(x_{n}\right)\right)$ is an Anti-state for $\models_{\mathfrak{A}}^{A}$.

Definition 3.9. We say $A \subseteq|\mathfrak{A}|$ demonstrates the observer effect under $\mathfrak{A}$ if and only if there exists a formula $\lambda$ and a variable assignment $s: \operatorname{Var} \rightarrow A$ with $\models_{\mathfrak{A}}^{A} \lambda[s]$ true, where at least one of the following holds:

i. There exists terminal terms $t_{1}, \ldots, t_{n}$ of $\lambda$, and there exists a constant free term $t\left(x_{1}, \ldots, x_{n}\right)$ where $\models_{\mathfrak{A}}^{A}\left(\lambda \wedge\left(t\left(t_{1}, \ldots, t_{n}\right)=t\left(t_{1}, \ldots, t_{n}\right)\right)\right)[s]$ doesn't have a truth value.

ii. There exists a terminal term $t$ of $\lambda$ and there exists an initial term $t^{\prime}$ of $t$, where $\models_{\mathfrak{A}}^{A}\left(\lambda \wedge\left(t^{\prime}=t^{\prime}\right)\right)[s]$ has no truth value.

Theorem 3.10. For $A \subseteq|\mathfrak{A}|$ the following are equivalent:

1. A demonstrates the observer effect under $\mathfrak{A}$.

2. A is not the point set of a substructure of $\mathfrak{A}$.

3. A has an Anti-state relative to $\mathfrak{A}$.

Proof. It is trivial to see that $(1) \Rightarrow(2) \Rightarrow(3)$. That $(3) \Rightarrow(1)$ can be seen as follows. Suppose $t\left(x_{1}, \ldots, x_{n}\right)$ is a term and $a_{1}, \ldots, a_{n} \in A$ so that $t^{\mathfrak{A}}\left(a_{1}, \ldots, a_{n}\right) \notin$ $A$. Taking $s: \operatorname{Var} \rightarrow A$ so that $s\left(x_{i}\right)=a_{i}$ and $\lambda$ to be the formula $\left(x_{1}=\right.$ $x_{1} \wedge x_{2}=x_{2} \wedge \ldots \wedge x_{n}=x_{n}$ ) we have $x_{1}, \ldots, x_{n}$ are the terminal terms of $\lambda$ where $\models_{\mathfrak{A}}^{A} \lambda[s]$ is true while $\models_{\mathfrak{A}}^{A}\left(\lambda \wedge\left(t\left(x_{1}, \ldots, x_{n}\right)\right)[s]\right.$ has no truth value.

Example 3.11. In this example we give a simple model of the observer effect as it occurs in the double slit experiment. We set up $\langle\ell, \mathfrak{A}\rangle$ and $S \subseteq|\mathfrak{A}|$ as follows: 
The non-logical symbols of $\ell$ will consist of a single unary function symbol $f$ and predicate symbols $E, P_{1}, P_{2}, D_{1}, D_{2}, D_{3}$.

The point set $|\mathfrak{A}|$ will be $\{1,2,3\} \times\{1,2,3\}$.

We will have for $n<3, m \leq 3, f^{\mathfrak{A}}(n, m)=(n+1, m)$ and for $n=3$ $f^{\mathfrak{A}}(n, m)=(n, m)$.

We will take $E^{\mathfrak{A}}=\{(1, m): m \in\{1,2,3\}\}, P_{1}^{\mathfrak{A}}=\{(2,1),(2,2)\}, P_{2}^{\mathfrak{A}}=$ $\{(2,3)\}$, and for $m \in\{1,2,3\}$ take $D_{m}^{\mathfrak{A}}=\{(3, m)\}$.

$$
S=\{(2,1),(2,3)\} \cup(\{1\} \times\{1,2,3\}) \cup(\{3\} \times\{1,2,3\})
$$

We give a model of the observer effect (Contextuality) on the double slit experiment as follows: The point set $|\mathfrak{A}|$ is meant to represent particles emitted from a source $E^{\mathfrak{A}}$, which move through slits $P_{1}^{\mathfrak{A}}, P_{2}^{\mathfrak{A}}$, and end at one of three detectors given by $D_{1}^{\mathfrak{A}}, D_{2}^{\mathfrak{A}}, D_{3}^{\mathfrak{A}}$. The function $f^{\mathfrak{A}}$ gives the transition of the particles from source to detectors.

When we only make an observation at the representative source $E$, and detectors $D_{1}, D_{2}, D_{3}$ we have the following for each $i \in\{1,2,3\}$ :

$$
\mid\left\{\left(f^{2}\right)^{\mathfrak{A}}(s(x)): s: \operatorname{Var} \rightarrow S \text { where } \models_{\mathfrak{A}}^{S}\left(E(x) \wedge D_{i}\left(f^{2}(x)\right)\right)[s]\right\} \mid=1
$$

Which more simply stated says that the number of points that can be observed at detector $D_{i}$ which transitioned there from a point observed at $E$ our source is 1 . This is a very loose approximation of the banding pattern associated with the double slit experiment. When we make the additional observation at the slits we get the following:

- $\mid\left\{\left(f^{2}\right)^{\mathfrak{A}}(s(x)): s: \operatorname{Var} \rightarrow S\right.$ where $\left.\models{ }_{\mathfrak{A}}^{S}\left(E(x) \wedge\left(P_{1}(f(x)) \vee P_{2}(f(x))\right) \wedge D_{1}\left(f^{2}(x)\right)\right)[s]\right\} \mid=1$

- $\mid\left\{\left(f^{2}\right)^{\mathfrak{A}}(s(x)): s: \operatorname{Var} \rightarrow S\right.$ where $\left.\models_{\mathfrak{A}}^{S}\left(E(x) \wedge\left(P_{1}(f(x)) \vee P_{2}(f(x))\right) \wedge D_{2}\left(f^{2}(x)\right)\right)[s]\right\} \mid=0$

- $\mid\left\{\left(f^{2}\right)^{\mathfrak{A}}(s(x)): s:\right.$ Var $\rightarrow S$ where $\left.\models S_{\mathfrak{A}}^{S}\left(E(x) \wedge\left(P_{1}(f(x)) \vee P_{2}(f(x))\right) \wedge D_{3}\left(f^{2}(x)\right)\right)[s]\right\} \mid=1$

Which gives that with the additional observation at the slits $P_{1}$, and $P_{2}$ of points transitioning from the source to the detectors, we have 1 point is detected at each detector $D_{1}$ and $D_{3}$, and no points are detected at $D_{2}$. If we take $D_{1}$ and $D_{3}$ to be directly behind the slits $P_{1}$ and $P_{2}$, and $D_{2}$ to sit between $D_{1}$, and $D_{3}$, then we see that the additional observation has caused us to detect points only directly behind the slits.

\subsection{A General Completeness Theorem For Anti-Structures}

Definition 3.12. We will call an Anti-Structure $\models_{\mathfrak{A}}^{A}$ codified if and only if given $\mathfrak{L}$ is the language of the structure $\mathfrak{A}$, and the set of constant symbols of $\mathfrak{L}$ is $\mathfrak{C}$, it holds that $A=\left\{c^{\mathfrak{A}}: c \in \mathfrak{C}\right\}$ where if $a, b \in \mathfrak{C}$ with $a \neq b$, then $a^{\mathfrak{A}} \neq b^{\mathfrak{A}}$. 
A codified structure $\mathfrak{A}$, is the codified Anti-structure $\models_{\mathfrak{A}}^{|\mathfrak{A}|}$.

When we have a codified Structure $\mathfrak{A}$ for a language $\mathfrak{L}$ having constant symbols C

- We will take $C V$ ar to be the set of variable to constant symbol assignments.

- For all variable assignments $v$ we will then have $c v \in C v a r$, so that for all variables $x, c v(x)$ is the constant where $c v(x)^{\mathfrak{A}}=v(x)$.

- For each formula $\phi$ and each $c v \in C v a r$ we will take $\phi[c v]$ to be the formula attained by substituting $c v(x)$ for $x$ in $\phi$ for each variable $x$ occurring in $\phi$.

- For each term $t$ of $\mathfrak{L}$ and each $c v \in C V a r$ we will take $t[c v]$ to be the term where the value of $c v(x)$ is substituted for $x$ into $t$ for each occurrence of $x$ in $t$ and each $x$ occurring in $t$. And given $m$ is a set of terms of $\mathfrak{L}$, then for all $c v \in C v a r$ we will have $m[c v]=\{t[c v] \mid t \in m\}$.

- If the set of constants of a language is designated by a symbol, say $S$ or $\mathfrak{C}$, then the set of variable to constant symbol functions will respectively be designated as $S V a r$ and $\mathfrak{C} V a r$. And for variable assignment $v$ we will substitute $s$ and $\mathfrak{c}$ for $c$ in $c v$ when we respectively have $S$ or $\mathfrak{C}$ in place of $C$ in the preceding.

The following is an example of a codified Anti-Structure.

Example 3.13. We will take our language to consist of a constant symbol $c_{n}$ for each $n \in \mathbb{Z}$ (integers), a binary function symbol + , for every $g: \mathbb{R} \rightarrow \mathbb{R}$ which is continuous (from and to the real line), a unary function symbol $f_{g}$, and the binary predicates $\geq$ and $=$. Our structure will be $\mathfrak{A}$ where $|\mathfrak{A}|=\mathbb{R}, c_{n}^{\mathfrak{A}}=n$, $f_{g}^{\mathfrak{A}}=g$ and $+{ }^{\mathfrak{A}}, \geq^{\overline{\mathfrak{A}}},={ }^{\mathfrak{A}}$ will have their usual meanings from $\mathbb{R}$. Taking $A=\mathbb{Z}$ we now have that $\models_{\mathfrak{A}}^{A}$ is a codified Anti-Structure. We note that an interesting attribute of $\models_{\mathfrak{A}}^{A}$ can be seen with the formulas:

- $\phi_{1}(x)$ equal to $\left[1 \geq f_{\cos \left(\frac{x \pi}{4}\right)}(x) \wedge\left(f_{x^{2}}\left(f_{\cos \left(\frac{x \pi}{4}\right)}(x)\right)+f_{x^{2}}\left(f_{\cos \left(\frac{x \pi}{4}\right)}(x)\right) \geq 1\right)\right]$

- $\phi_{2}(x)$ equal to $\left[f_{x^{2}}\left(f_{\cos \left(\frac{x \pi}{4}\right)}(x)\right)+f_{x^{2}}\left(f_{\cos \left(\frac{x \pi}{4}\right)}(x)\right) \geq 1\right]$

Where then for $v_{1}(x)=0$ and $v_{2}(x)=1$ we have:

- $\models_{\mathfrak{A}}^{A} \phi_{1}\left[v_{1}\right]$ is true, while $\models_{\mathfrak{A}}^{A} \phi_{1}\left[v_{2}\right]$ has no meaning.

- $\models_{\mathfrak{A}}^{A} \phi_{2}\left[v_{1}\right]$ and $\models_{\mathfrak{A}}^{A} \phi_{2}\left[v_{2}\right]$ are true.

Abusing notation by substituting $g$ for $f_{g}$ we might more clearly see the meaning of the above two bullets if we state them as follows:

i. Under $\models_{\mathfrak{A}}^{A}$ we have $\left[1 \geq \cos (0) \wedge\left(\cos (0)^{2}+\cos (0)^{2} \geq 1\right)\right]$ is true while $\left[1 \geq \cos \left(\frac{\pi}{4}\right) \wedge\left(\cos \left(\frac{\pi}{4}\right)^{2}+\cos \left(\frac{\pi}{4}\right)^{2} \geq 1\right)\right]$ has no meaning. 
ii. Under $\models_{\mathfrak{A}}^{A}$ we have both $\left[\cos (0)^{2}+\cos (0)^{2} \geq 1\right]$ and $\left[\cos \left(\frac{\pi}{4}\right)^{2}+\cos \left(\frac{\pi}{4}\right)^{2} \geq 1\right]$ are true.

Where clearly i. and ii. are a consequence of the only formulas in $\models_{\mathfrak{A}}^{A}$ having meaning being the ones that have terminal terms which are integer number values. Taking $\phi_{1}$ and $\phi_{2}$ to have terminal terms which represent measured values, then we would have that their truth under a variable assignment is only discernible when the measured values that they are statements about, have natural number values. For further comment on the connection between the above example and the use of the Plank constant please see Comment 2 in Section 5 .

This section pertains to what we call the general completeness theorem for codified Anti-Structures. We specify that this is a general notion of completeness because even though it does specify the collections of formulas that can be modeled by a codified Anti-Structure and even though the properties on these collections of formulas(Systems) should apply to formulas generated from any real world experiment, what it does not do is clearly define a process for taking real world experiments and then deriving an Anti-Structure model of the experiment. It is in Section 4 that we go over process for generating Anti-Structure models for experiments as well as the Anti-Structure model for QED. We now proceed with our set up for our general completeness theorem.

Definition 3.14. Given $\mathfrak{L}$ is a formal language and $\mathfrak{C}$ is the set of constant symbols of $\mathfrak{L}$, we have $Y \subseteq\{\phi: \phi$ is a variable free formula of $\mathfrak{L}\}$ is a system for $\mathfrak{L}$ if and only if all of the following hold:

1. For all $c_{1}, c_{2} \in \mathfrak{C}$ we have $\left(c_{1}=c_{2}\right) \in Y$ if and only if $c_{1}$ and $c_{2}$ are the same constant symbol.

2. For all $\phi \in Y$ if $t$ is a terminal term of $\phi$, then $\exists c \in \mathfrak{C}$ where $(t=c) \in Y$.

3. If $Y \models \psi$ where $\psi$ is a variable free formula of $\mathfrak{L}$ and for all terminal terms $t$ of $\psi$ there exists a $c \in \mathfrak{C}$ where $(t=c) \in Y$, then $\psi \in Y$.

4. For all $n$-ary predicate symbols $P$ if for $c_{1}, \ldots, c_{n} \in \mathfrak{C}, P\left(c_{1}, \ldots, c_{n}\right) \notin Y$, then $\neg P\left(c_{1}, \ldots, c_{n}\right) \in Y$.

5. If $\left\{\phi\left(t_{1}\right),\left(t_{1}=t_{2}\right)\right\} \subseteq Y$, then $\phi\left(t_{2}\right) \in Y$.

Remark 3.15. We note that if a system $Y$ is given, then $Y$ is a consistent set of formulas, since $Y$ being inconsistent would yield that $Y \models(a=b)$ for distinct constant symbols $a$ and $b$ and then 3 above would yield $(a=b) \in Y$ which would contradict requirement 1.

Proposition 3.16. Given $\models_{\mathfrak{A}}^{A}$ is a codified Anti-Structure with language $\mathfrak{L}$, then both of the following hold:

I. $Y=\left\{\psi \mid \psi\right.$ is a variable free formula of $\mathfrak{L}$ and $\models_{\mathfrak{A}}^{A} \psi$ is true $\}$ yields $Y$ is a system. 
II. If $Y$ is as given above and $\models_{\mathfrak{B}}^{B}$ is a codified Anti-structure also having language $\mathfrak{L}$ where $Y=\left\{\psi \mid \psi\right.$ is a variable free formula of $\mathfrak{L}$ and $\models_{\mathfrak{B}}^{B} \psi$ is true $\}$, then for all sentences $\psi, \models_{\mathfrak{A}}^{A} \psi$ agrees with $\models_{\mathfrak{B}}^{B} \psi$.

Before we prove the above proposition we note that it is saying that codified Anti-Structures give a system and that a system given for a codified Antistructure uniquely identifies the Anti-structure.

Proof. Taking $\mathfrak{C}$ to be the constant symbols of $\mathfrak{L}$ where $A=\left\{c^{\mathfrak{A}} \mid c \in \mathfrak{C}\right\}$, the proof of part (I) of the proposition can be given directly by noting that $Y=$ $\left\{\psi \mid \psi\right.$ is a variable free formula where $\left.\operatorname{ter}(\psi)^{\mathfrak{A}}\right) \subseteq A$ and $\models_{\mathfrak{A}} \psi$ is true $\}$ where then we have:

- Requirement 1 for $Y$ being a system follows from the fact that for all $c \in \mathfrak{C}, \operatorname{ter}(c=c)^{\mathfrak{A}}=\left\{c^{\mathfrak{A}}\right\} \subset A$ and we have $\models_{\mathfrak{A}}(c=c)$ is true.

- Requirement 2 for a system follows from the fact that if $\phi \in Y$ and if $t$ is a terminal term of $\phi$, then $t^{\mathfrak{A}} \in A$ and therefore there exists a $c \in \mathfrak{C}$ where $t^{\mathfrak{A}}=c^{\mathfrak{A}}$ and consequently $\models_{\mathfrak{A}}(c=t)$ is true and we have $(c=t) \in Y$.

- Requirements 3, 4 and 5 for $Y$ to be a system follow directly from the fact that $Y=\left\{\psi \mid \psi\right.$ is a quantifier free sentence where $\operatorname{ter}(\psi)^{\mathfrak{A}} \subseteq A$ and $\models_{\mathfrak{A}} \psi$ is true $\}$.

The proof of part (II) of the above follows from the subsequent lemma.

Lemma 3.17. Given $\mathfrak{A}_{1}$ and $\mathfrak{A}_{2}$ are structures for a language $\mathfrak{L}$ and $A \subseteq$ $\left|\mathfrak{A}_{1}\right| \cap\left|\mathfrak{A}_{2}\right|$ the following are equivalent:

- $\models_{\mathfrak{A}_{1}}^{A} \phi[s]$ agrees with $\models_{\mathfrak{A}_{2}}^{A} \phi[s]$ for all formulas $\phi$ and all variable assignments $s: \operatorname{Var} \rightarrow A$.

- For all $n$-ary predicates $P, P^{\mathfrak{A}_{1}} \cap A^{n}=P^{\mathfrak{A}_{2}} \cap A^{n}$ and for all terms $t$ and for all $a_{1}, \ldots, a_{n} \in A$, we have if $t^{\mathfrak{A}_{1}}\left(a_{1}, \ldots, a_{n}\right) \in A$ or $t^{\mathfrak{A}_{2}}\left(a_{1}, \ldots, a_{n}\right) \in A$, then $t^{\mathfrak{A}_{1}}\left(a_{1}, \ldots, a_{n}\right)=t^{\mathfrak{A}_{2}}\left(a_{1}, \ldots, a_{n}\right)$. Note here we include $=$ as a binary predicate symbol.

Proof. We give a proof of the non-trivial direction.

Given $\mathfrak{A}_{1}$ and $\mathfrak{A}_{2}$ are structures for $\mathfrak{L}$ and $A \subseteq\left|\mathfrak{A}_{1}\right| \cap\left|\mathfrak{A}_{2}\right|$, suppose we have:

1. For each $n$-ary predicate $P, P^{\mathfrak{A}_{1}} \cap A^{n}=P^{\mathfrak{A}_{2}} \cap A^{n}$.

2. For each term $t$ and all points $a_{1}, \ldots, a_{n} \in A$ if $t^{\mathfrak{A}_{1}}\left(a_{1}, \ldots, a_{n}\right) \in A$ or $t^{\mathfrak{A}_{2}}\left(a_{1}, \ldots, a_{n}\right) \in A$, then $t^{\mathfrak{A}_{1}}\left(a_{1}, \ldots, a_{n}\right)=t^{\mathfrak{a}_{2}}\left(a_{1}, \ldots, a_{n}\right)$.

We give a proof by induction that for quantifier free formulas $\phi$ and variable assignments $s: \operatorname{Var} \rightarrow A$ we have $\models_{\mathfrak{A}_{1}}^{A} \phi[s]$ agrees with $\models_{\mathfrak{A}_{2}}^{A} \phi[s]$ and note that once we have shown this we will have that for all formulas $\psi$ and all variable assignments $s: \operatorname{Var} \rightarrow A, \models_{\mathfrak{A}_{1}}^{A} \psi[s]$ agrees with $\models_{\mathfrak{A}_{2}}^{A} \psi[s]$.

Base Case. Suppose $P\left(x_{1}, \ldots, x_{n}\right)$ is a predicate, $t_{1}, \ldots, t_{n}$ are terms and $s: \operatorname{Var} \rightarrow A$. 
It follows for an $i \in\{1,2\}$ we have $\models_{\mathfrak{A}_{i}}^{A} P\left(t_{1}, \ldots, t_{n}\right)[s]$ has no truth value if and only if there exists a $j \in\{1, \ldots, n\}$ where $t_{j}^{\mathfrak{A}_{i}}[s] \notin A$ and therefore as a consequence of our assumption 2 we would have $t_{j}^{\mathfrak{A}_{1}}[s] \notin A$ and $t_{j}^{\mathfrak{A}_{2}}[s] \notin A$. It follows from the preceding that we have: $\left(\models_{\mathfrak{A}_{1}}^{A} P\left(t_{1}, \ldots, t_{n}\right)[s]\right.$ has no truth value) $\Leftrightarrow$ ( there exists a $j \in\{1, \ldots, n\}$ where $\left.t_{j}^{\mathfrak{A}_{1}}[s] \notin A\right) \Leftrightarrow($ there exists a $j \in\{1, \ldots, n\}$ where $\left.t_{j}^{\mathfrak{A}_{2}}[s] \notin A\right) \Leftrightarrow\left(\models_{\mathfrak{A}_{2}}^{A} P\left(t_{1}, \ldots, t_{n}\right)[s]\right.$ has no truth value $)$.

It follows from the above that: $\left(\models_{\mathfrak{A}_{1}}^{A} P\left(t_{1}, \ldots, t_{n}\right)[s]\right.$ has a truth value $)$ $\Leftrightarrow\left(\mid={ }_{\mathfrak{A}_{2}}^{A} P\left(t_{1}, \ldots, t_{n}\right)[s]\right.$ has a truth value $) \Leftrightarrow$ (for all $j \in\{1, \ldots, n\}$ we have $t_{j}^{\mathfrak{A}_{1}}[s]=t_{j}^{\mathfrak{A}_{2}}[s] \in A$ ) (this follows from our assumption 2). Since $P^{\mathfrak{A}_{1}} \cap A^{n}=P^{\mathfrak{A}_{2}} \cap A$ it follows if for all $j \in\{1, \ldots, n\}$ we have $t_{j}^{\mathfrak{A}_{1}}[s]=$ $t_{j}^{\mathfrak{A}_{2}}[s] \in A$, then $\left\langle t_{j}^{\mathfrak{A}_{1}}[s], \ldots t_{j}^{\mathfrak{A}_{1}}[s]\right\rangle \in P^{\mathfrak{A}_{1}} \Leftrightarrow\left\langle t_{j}^{\mathfrak{A}_{2}}[s], \ldots t_{j}^{\mathfrak{A}_{2}}[s]\right\rangle \in P^{\mathfrak{A}_{2}}$ and consequently given for all $j \in\{1, \ldots, n\}, t_{j}^{\mathfrak{A}_{1}}[s]=t_{j}^{\mathfrak{A}_{2}} \in A$ we have $\models_{\mathfrak{A}_{1}}^{A} P\left(t_{1}, \ldots, t_{n}\right)[s] \Leftrightarrow \models_{\mathfrak{A}_{2}}^{A} P\left(t_{1}, \ldots, t_{n}\right)[s]$.

We note that given the above it is trivial to see that $\models_{\mathfrak{A}_{1}}^{A} \neg P\left(t_{1}, \ldots, t_{n}\right)[s]$ agrees with $\models_{\mathfrak{R}_{2}}^{A} \neg P\left(t_{1}, \ldots, t_{n}\right)[s]$.

We assume $\phi\left(x_{1}, \ldots, x_{n}\right)$ and $\psi\left(y_{1}, \ldots, y_{m}\right)$ are quantifier free function symbol free formulas where for all terms $t_{1}, \ldots, t_{n}, r_{1}, \ldots, r_{m}$ we have for all $s: \operatorname{Var} \rightarrow A$ that $\models_{\mathfrak{A}_{1}}^{A} \phi\left(t_{1}, \ldots, t_{n}\right)[s]$ agrees with $\models_{\mathfrak{A}_{2}}^{A} \phi\left(t_{1}, \ldots, t_{n}\right)[s]$ and $\models_{\mathfrak{R}_{1}}^{A} \psi\left(r_{1}, \ldots, r_{m}\right)[s]$ agrees with $\models_{\mathfrak{R}_{2}}^{A} \psi\left(r_{1}, \ldots, r_{m}\right)[s]$.

We take $s: \operatorname{Var} \rightarrow A$ and $t_{1}, \ldots, t_{n}, r_{1}, \ldots, r_{m}$ to be terms.

Let $\gamma \in\left\{\phi\left(t_{1}, \ldots, t_{n}\right) \wedge \psi\left(r_{1}, \ldots, r_{m}\right), \phi\left(t_{1}, \ldots, t_{n}\right) \vee \psi\left(r_{1}, \ldots, r_{m}\right), \phi\left(t_{1}, \ldots, t_{n}\right) \rightarrow\right.$ $\left.\psi\left(r_{1}, \ldots, r_{m}\right)\right\}$.

It follows for $i \in\{1,2\}$ that $\models_{\mathfrak{A}_{i}}^{A} \gamma[s]$ is undefined if and only if there exists an $l \in\left\{t_{1}, \ldots, t_{n}, r_{1}, \ldots, r_{m}\right\}$ where $l^{\mathfrak{A}_{i}}[s] \notin A$ and consequently $l^{\mathfrak{A}_{1}}[s], l^{\mathfrak{A}_{2}}[s] \notin A$ which yields both $\models_{\mathfrak{A}_{1}}^{A} \gamma[s]$ and $\models_{\mathfrak{A}_{2}}^{A} \gamma[s]$ have no truth value.

It follows from the preceding that:

$\left(\models_{\mathfrak{A}_{1}}^{A} \gamma[s]\right.$ has a truth value or $\models_{\mathfrak{A}_{2}}^{A} \gamma[s]$ has a truth value $)$

$\Leftrightarrow$

(for all $l \in\left\{t_{1}, \ldots, t_{n}, r_{1}, \ldots, r_{m}\right\}$ we have $l^{\mathfrak{A}_{1}}[s]=l^{\mathfrak{A}_{2}}[s] \in A$ )

$\Leftrightarrow$

$\left(\models_{\mathfrak{A}_{1}}^{A} \gamma[s]\right.$ and $\models_{\mathfrak{A}_{2}}^{A} \gamma[s]$ have a truth values $)$

$\Leftrightarrow$

$\left(\models_{\mathfrak{A}_{1}}^{A} \phi\left(t_{1}, \ldots, t_{n}\right)[s]\right.$ has the same truth value as $\models_{\mathfrak{A}_{2}}^{A} \phi\left(t_{1}, \ldots, t_{n}\right)[s]$ and $\models_{\mathfrak{A}_{1}}^{A} \psi\left(r_{1}, \ldots, r_{m}\right)[s]$ has the same truth value as $\left.\models_{\mathfrak{A}_{2}}^{A} \psi\left(r_{1}, \ldots, r_{m}\right)[s]\right)$

$\Leftrightarrow$

$\left(\models{ }_{\mathfrak{A}_{1}}^{A} \gamma[s]\right.$ has the same truth value as $\left.\models_{\mathfrak{A}_{2}}^{A} \gamma[s].\right)$

We now give our general completeness theorem for codified Anti-Structures. 
Theorem 3.18. Given $Y$ is a collection of variable free sentences for a language $\mathfrak{L}$, we have $Y$ is a system if and only if there exists a codified AntiStructure $\models_{\mathfrak{A}}^{A}$ for $\mathfrak{L}$, where

$$
Y=\left\{\phi: \phi \text { is a quantifier free sentence of } \mathfrak{L} \text { where } \models_{\mathfrak{A}}^{A} \phi\right\}
$$

Proof. We argue the non trivial direction of the theorem. Suppose $Y$ is a system for a language $\mathfrak{L}$ having constant symbols $\mathfrak{C}$. Note there does not exist a predicate $P$ and constant symbols $c_{1}, \ldots, c_{n} \in \mathfrak{C}$ with $P\left(c_{1}, \ldots, c_{n}\right) \wedge$ $\neg P\left(c_{1}, \ldots, c_{n}\right) \in Y$.

We define classes for the set of variable free terms of $\mathfrak{L}$ so that for a term $t$ the class of $t$ will be $\bar{t}=\{r: r$ is a variable free term of $\mathfrak{L}$ and $Y \models(t=r)\}$. To address the slight deviation with the stated definition of system, in this argument we note: if we replace $Y \models(t=r)$ in the above with the equivalence relation $t \sim r$ iff either:

$(t=r) \in Y$ or

there exists a term $q\left(x_{1}, \ldots, x_{n}\right)$ (where $x_{1}, \ldots, x_{n}$ may not occur in $q$ ), and for $i \leq n$ there exists $\left(g_{i}=h_{i}\right) \in Y$, so that $t$ is syntactically the term $q\left(g_{1}, \ldots, g_{n}\right)$ and $r$ is syntactically the term $q\left(h_{1}, \ldots, h_{n}\right)$,

then we will have:

for any term $t$ and constant $c, t \sim c$ iff $(t=c) \in Y$

and taking $\bar{t}$ to be the equivalence class for $t$ under $\sim$ and substituting this change into the construction of $\mathfrak{A}$ below, it is easily checked that a structure for $Y$ is given.

Since the above $\sim$ gives a structure for $Y$ where for any constant $c$ and any term $t,(t=c)$ is true in the structure iff $(t=c) \in Y$, it follows $Y \models(t=c)$ iff $(t=c) \in Y$.

We take $\mathfrak{A}$ to be the following structure:

$|\mathfrak{A}|=\{\bar{t}: t$ is a variable free term of $\mathfrak{L}\}$.

For each constant symbol $c$ we will have $c^{\mathfrak{A}}=\bar{c}$.

For each function symbol $f$ we will have $f^{\mathfrak{A}}\left(\bar{r}_{1}, \ldots, \bar{r}_{n}\right)=\overline{f\left(r_{1}, \ldots, r_{n}\right)}$.

For $n$-ary predicate $P$ we will have $\left\langle\bar{t}_{1}, \ldots, \bar{t}_{n}\right\rangle \in P^{\mathfrak{A}}$ if and only if $P\left(t_{1}, \ldots, t_{n}\right) \in Y$.

We note $\mathfrak{A}$ gives us a structure if and only if

for all n-ary function symbols $f$ and all n-ary predicate symbols $P$, given variable free terms $t_{1}, \ldots, t_{n}$, we have if $Y \models\left(t_{i}^{\prime}=t_{i}\right)$ for $i \leq n$, then

$-Y \models\left(f\left(t_{1}, \ldots, t_{n}\right)=f\left(t_{1}^{\prime}, \ldots, t_{n}^{\prime}\right)\right)$, so that $f^{\mathfrak{A}}\left(\overline{t_{1}}, \ldots, \overline{t_{n}}\right)=\overline{f\left(t_{1}, \ldots, t_{n}\right)}$ is a well defined function. 
- $P\left(t_{1}, . ., t_{n}\right) \in Y$ if and only if $P\left(t_{1}^{\prime}, \ldots, t_{n}^{\prime}\right) \in Y$, so that $P^{\mathfrak{A}}$ is a well defined subset of $|\mathfrak{A}|^{n}$.

We now take $A=\{\bar{c}: c \in \mathfrak{C}\}$ and show that

$Y=\left\{\phi: \phi\right.$ is a quantifier free sentence of $\mathfrak{L}$ where $\left.\models_{\mathfrak{A}}^{A} \phi\right\}$ by noting the following:

i. For function free quantifier free formula $\phi\left(x_{1}, \ldots, x_{n}\right)$ and all variable free terms $t_{1}, \ldots, t_{n}$ we have $\left(\models_{\mathfrak{A}}^{A} \phi\left(t_{1}, \ldots, t_{n}\right)\right.$ has a truth value $) \Leftrightarrow($ there exists $c_{1}, \ldots, c_{n} \in \mathfrak{C}$ where for $\left.i \in\{1, \ldots, n\},\left(t_{i}=c_{i}\right) \in Y\right)$.

ii. For all $n$-ary predicates $P$ and all variable free terms $t_{1}, \ldots, t_{n}$ we have $\left(\models_{\mathfrak{A}}^{A} P\left(t_{1}, \ldots, t_{n}\right)\right.$ is true $) \Leftrightarrow\left(P\left(t_{1}, \ldots, t_{n}\right) \in Y\right)$.

iii. It follows from parts 2,3 and 4 of Definition 3.14 and parts (i) and (ii) of this note that under the assumption that $\models_{\mathfrak{A}}^{A} P\left(t_{1}, \ldots, t_{n}\right)$ has a truth value we may take $\left(t_{i}=c_{i}\right) \in Y$ for some $c_{i} \in \mathfrak{C}$ and we have $\left(\models_{\mathfrak{A}}^{A} P\left(t_{1}, \ldots, t_{n}\right)\right.$ is false $) \Leftrightarrow\left(P\left(t_{1}, \ldots, t_{n}\right) \notin Y\right) \Leftrightarrow\left(P\left(c_{1}, \ldots, c_{n}\right) \notin Y\right)$ $\left.\Leftrightarrow\left(\neg P\left(c_{1}, \ldots, c_{n}\right) \in Y\right) \Leftrightarrow\left(\neg P\left(t_{1}, \ldots, t_{n}\right) \in Y\right)\right)$.

iv. Since $\left\{\neg P\left(t_{1}, \ldots, t_{n}\right), P\left(t_{1}, \ldots, t_{n}\right)\right\} \subseteq Y$ would yield $Y$ is not consistent, which is in contradiction with the definition of $Y$, it follows if $\neg P\left(t_{1}, \ldots, t_{n}\right) \in Y$, then $P\left(t_{1}, \ldots, t_{n}\right) \notin Y$. It follows from the preceding that if $\neg P\left(t_{1}, \ldots, t_{n}\right) \in Y$, then there exists $c_{1}, \ldots, c_{n} \in \mathfrak{C}$ where $\left(t_{i}=c_{i}\right) \in Y$ and consequently $\models_{\mathfrak{A}}^{A} P\left(t_{1}, \ldots, t_{n}\right)$ has a truth value where $P\left(t_{1}, \ldots, t_{n}\right) \notin Y$ yields $\models_{\mathfrak{A}}^{A} \neg P\left(t_{1}, \ldots, t_{n}\right)$ is true.

v. From (i-iv) of this note we have

$* Y \cap\left\{P\left(t_{1}, \ldots, t_{n}\right): t_{1}, \ldots, t_{n}\right.$ are variable free terms and $P$ is a predicate symbol $\}$ $=\left\{P\left(t_{1}, \ldots, t_{n}\right): t_{1}, \ldots, t_{n}\right.$ are variable free terms and $\models_{\mathfrak{A}}^{A} P\left(t_{1}, \ldots, t_{n}\right)$ is true $\}$.

* $Y \cap\left\{\neg P\left(t_{1}, \ldots, t_{n}\right): t_{1}, \ldots, t_{n}\right.$ are variable free terms and $P$ is a predicate symbol $\}$ $=\left\{\neg P\left(t_{1}, \ldots, t_{n}\right): t_{1}, \ldots, t_{n}\right.$ are variable free terms and $\models_{\mathfrak{A}}^{A} \neg P\left(t_{1}, \ldots, t_{n}\right)$ is true $\}$

vi. If $\phi$ is a variable free formula, then taking

$\Delta_{1}=\left\{P\left(t_{1}, \ldots, t_{n}\right): P\left(t_{1}, \ldots, t_{n}\right)\right.$ occurs in $\phi$ and $\models_{\mathfrak{A}}^{A} P\left(t_{1}, \ldots, t_{n}\right)$ is true $\}$ and

$\Delta_{2}=\left\{\neg P\left(t_{1}, \ldots, t_{n}\right): P\left(t_{1}, \ldots, t_{n}\right)\right.$ occurs in $\phi$ where $\models_{\mathfrak{A}}^{A} \neg P\left(t_{1}, \ldots, t_{n}\right)$ is true $\}$.

From Remark 3.4 we have

$\left(\models_{\mathfrak{A}}^{A} \phi\right.$ is true $) \Leftrightarrow$

$\left(\{\bar{t}: t \in \operatorname{ter}(\phi)\} \subseteq A\right.$ and $\left.\models_{\mathfrak{A}} \phi\right) \Leftrightarrow$

$\left(\{\bar{t}: t \in \operatorname{ter}(\phi)\} \subseteq A\right.$ and $\left.\models_{\mathfrak{A}} \bigwedge\left(\Delta_{1} \cup \Delta_{2}\right)\right)$.

It follows

$\left(\models_{\mathfrak{A}}^{A} \phi\right.$ is true $) \Leftrightarrow\left(\{t=t: t \in \operatorname{ter}(\phi)\} \subseteq Y\right.$ and $\left.\Delta_{1} \cup \Delta_{2} \models \phi\right)$ and from (v) of this note we also have $\Delta_{1} \cup \Delta_{2} \subset Y$. From part 3 of Definition 3.14 we conclude that $\left(\models_{\mathfrak{A}}^{A} \phi\right.$ is true $) \Leftrightarrow(\phi \in Y)$. We note that in the preceding, we make use of the fact that $Y$ is consistent and that we have either $\left(\Delta_{1} \cup \Delta_{2}\right) \models \phi$ or $\left(\Delta_{1} \cup \Delta_{2}\right) \models \neg \phi$. 
Remark 3.19. We point out that in the above $A=\{\bar{c} \mid c \in C\}$ along with $f^{\mathfrak{A}}\left\lceil A\right.$ and $P^{\mathfrak{A}} \cap A^{n}$ (for n-ary predicate $P$ ) are all definable from $Y$ without having to define $\mathfrak{A}$ (noting that $f^{\mathfrak{A}}\lceil A$ is a partial function). We then have that we do not need $|\mathfrak{A}|-A$ to define truth for our Anti-Structure, so that our Anti-states represent no tangible points but rather are no information in an absolute sense (they are naming nothing, and the Anti-Structure makes no statements true or false about them). If a system $Y$ is taken as an absolute record of everything that could possibly be observed, then the preceding gives that we can give our Anti-Structure from only the observed values, leaving terms undefined (and thus in an Anti-state) if $Y$ has no record of them having a value.

Before we give our first model completeness theorem we must give some formal definitions which will allow us to have a mathematical notion of experiment.

Definition 3.20. Given $L$ is a first order language having set of constant symbols $C$ and set of variable symbols $V$, and given $\kappa$ is a cardinal, we define a $\kappa$-contextual model for $L$ as follows:

- For $i \in \kappa$ an $m_{i} \subseteq\{t \mid t$ is a constant free term of $L$ and $t$ is not a variable $\}$ where $\left|m_{i}\right|<\omega_{0}$ and for $i \neq j, m_{i} \neq m_{j}$. We call each $m_{i}$ a set of measurables for the $\kappa$-contextual model.

- We define

$$
F_{i}=\left\{\phi \mid \phi \text { is a quantifier free formula of } L \text { and } \operatorname{ter}(\phi)-(C \cup V)=m_{i}\right\}
$$

- For $i \in \kappa$ a structure $\mathfrak{A}_{i}$ for $L$ is selected.

- Let $\operatorname{Var} A_{i}$ denote the set of variable assignments for $\mathfrak{A}_{i}$.

- Contx : $\bigcup_{i \in \kappa}\left(F_{i} \times \operatorname{Var} A_{i}\right) \rightarrow\{$ true, false $\}$ such that for $\langle\phi, v\rangle \in F_{i} \times \operatorname{Var}_{i}$ we have $\operatorname{Contx}(\phi, v)=\left(\models_{\mathfrak{A}_{i}} \phi[v]\right)$.

Definition 3.21. Given $L$ is a first order language having the set of constant symbols $C$ and the set of variable symbols $V$, and given $m$ is a collection of constant free terms of $L$, if $\mathfrak{A}$ is a codified structure for the language $L$ where $r$ is a variable free term of $L$, then $r$ will be constructable from $m$ under $\mathfrak{A}$ if and only if given $Y_{0}=\left\{\phi[c v] \mid \operatorname{ter}(\phi)-(C \cup V) \subseteq m\right.$ and $\models_{\mathfrak{A}} \phi[c v]$ is true $\}$ and $Y_{n+1}=\left\{\phi(t) \mid \exists\left\{\phi\left(t^{\prime}\right),\left(t^{\prime}=t\right)\right\} \subseteq Y_{n}\right\}$, then there exists a $c \in C$ where $(r=c) \in \bigcup_{n \in \mathbb{N}} Y_{n}$. We will denote the set of constructable terms from $m$ under $\mathfrak{A}$ as $\operatorname{Con}(m, \mathfrak{A})$.

Definition 3.22. Contx is a $\kappa$-simple contextual model, if and only if the following hold: 
i. Contx is a $\kappa$-contextual model for a language $L$. We take constant symbols of $L$ as being given in an enumeration, where $c_{\alpha}$ denotes the $\alpha$ 'th constant symbol in the enumeration, and $\mathcal{I}$ is the index set for the enumeration. We will assume $L$ represents the language of Contx for our remaining requirements.

ii. For all $i \in \kappa$, if $\left|\mathfrak{A}_{i}\right| \neq \varnothing$, then $\left|\mathfrak{A}_{i}\right|=\left\{c_{\alpha}^{\mathfrak{A}_{i}} \mid \alpha \in \mathcal{I}\right\}$. And for all $i \in \kappa$, if $\left|\mathfrak{A}_{i}\right| \neq \varnothing$, then $\models_{\mathfrak{A}_{i}} c_{l} \neq c_{j}$ is true for each $l \neq j$ from our index.

iii. For all predicates $P$ of the language $L$, if $P$ is an m-ary predicate, then for all $\left\langle a_{1}, \ldots, a_{n}\right\rangle \in\left\{c_{\alpha} \mid \alpha \in \mathcal{I}\right\}^{n},\left(\models_{\mathfrak{A}_{i}} P\left(a_{1}, \ldots, a_{n}\right)\right)=\left(\models_{\mathfrak{A}_{j}} P\left(a_{1}, \ldots, a_{n}\right)\right)$ for all $i, j$.

iv. We have

$$
\Delta_{i}=\left\{\phi[c v] \mid \phi \in F_{i}, c v \in C V a r \text { and }\left(\models_{\mathfrak{A}_{i}} \phi[c v]\right) \text { is true }\right\}
$$

v. We now require that if there exists a $c v \in C V a r$ where $t[c v] \in m_{i}[c v] \cap$ $\operatorname{Con}\left(m_{j}, \mathfrak{A}_{j}\right)$, for $i \neq j$ and it is not the case that there exists $r[c v] \in m_{i}[c v]$ where:

$a_{1} . r[c v] \notin \operatorname{Con}\left(m_{j}, \mathfrak{A}_{j}\right)$,

$a_{2}$. and $r[c v]$ shares a sub-term with $t[c v]$ so that we might have $(r[c v]$ is a sub-term of $t[c v])$ or $(t[c v]$ is a sub-term of $r[c v])$,

, then there exists a $b \in C$ where we have $t^{\mathfrak{A}_{i}}[c v]=b^{\mathfrak{A}_{i}}$ and $t^{\mathfrak{A}_{j}}[c v]=b^{\mathfrak{A}_{j}}$.

When $\kappa$ does not need to be explicitly named a $\kappa$-simple contectual model may be refered to as a simple contextual model.

Definition 3.23. An experiment will be said to have a simple contextual model if and only if

- The experiment has a first order language $L$ describing all measurements and outcomes of measurements made in the experiment. The set of constants of $L$ will be $C$ and the set of variables for $L$ will be $V$.

- The measurements of the experiment are described by formulas $\phi_{i}$ for $i \leq n \in \mathbb{N}$ and $m_{i}=\operatorname{ter}\left(\phi_{i}\right)-(C \cup V)$. The act of measurement and its outcome is represented by checking to see if $\phi_{i}[c v]$ is true for $c v \in C V a r$. The semantics here is that $c v$ is setting the variables of $\phi_{i}$ to constants represents a selection of initial and final conditions for the experiment and then we observe the real world representations of the terms of $\phi_{i}$ under these conditions to determine if $\phi_{i}[c v]$ is true.

- There exists a simple contextual model Contx for $L$ and $m_{1}, \ldots, m_{n}, \ldots$ where $\Delta_{i}$ is a collection of quantifier free statements which the experiment gives as true under the measurement given by the terminal terms of $\phi$. We have that if there exists a $\phi$ where ter $(\phi)-(C \cup V)=m_{i}$ and a $c v \in C V a r$ where $\psi$ equals $\phi[c v]$ and $\psi$ is given as true by our experiment when we are looking at $m_{i}[c v]$ (see the preceding bullet point), then $\psi \in \Delta_{i}$. 
In order to give our first model completeness theorem we must define a $\pi$ map for the formulas of a simple contextual model. We choose $\pi$ here because of the projection like nature of these Anti-Structure models.

Definition 3.24. Given $L$ is a first order language with constant symbols $C$, then $\pi \phi \in \pi$ Form(L) if and only if there exists a formula $\phi$ of $L$ where:

- For each $c \in C$ we have a variable $z_{c} \notin L$. We take $\operatorname{cns}(\phi)$ to be the set of constants from $C$ which occur in $\phi$ and we take $\phi^{\prime}$ to be the formula where $\operatorname{cns}\left(\phi^{\prime}\right)=\varnothing$ and replacing each occurrence of $z_{c}$ in $\phi^{\prime}$ with $c$ gives $\phi$.

$$
\text { - } \phi^{\prime \prime}=\bigwedge_{c \in \operatorname{cns}(\phi)}\left(z_{c}=c\right)
$$

- Given $\operatorname{cns}(\phi)=\left\{c_{0}, \ldots, c_{n}\right\}$ we take $\phi^{*}=\exists z_{c_{0}}, \ldots, \exists z_{c_{n}}\left(\phi^{\prime \prime} \wedge \phi^{\prime}\right)$.

- We have a unary function symbol $\pi \notin L$ where then $\pi \phi$ is the formula given by replacing each terminal term $t$ of $\phi^{*}$ with $\pi(t)$. Note, that the variable $x$ in $\forall x$ and $\exists x$ is not an occurrence of a terminal term and this will hold for all variables (and does not proclude them from being terminal terms).

We will take $\pi L$ to be the language of $\pi$ Form(L)

Theorem 3.25. First Model Completeness Theorem

Given Contx gives a $\kappa$ - simple contextual model for a language $L$ having the set of constants $C$, variable set $V$, and for $i \in \kappa$ a set of measurables $m_{i}$, there exists an Anti-Structure $\models_{\mathfrak{A}}^{A}$ for a language which includes $\pi L$, where for all $i \in \kappa$, for all $\phi$ with $\operatorname{ter}(\phi)-(C \cup V)=m_{i}$ and all variable assignments $v \in \operatorname{Var} A_{i}$, we have $\operatorname{Contx}(\phi, v)=\left(\models_{\mathfrak{A}}^{A} \pi(\phi[c v])\right)$. Note that $c v$ was given in definition 3.12.

Proof. Since Contx is a $\kappa$-simple contextual model it follows it is also a $\kappa$ contextual model and therefore for each $m_{i}$ we have a structure $\mathfrak{A}_{i}$ where:

- For all $i \in \kappa$ if $\left|\mathfrak{A}_{i}\right| \neq \varnothing$, then $\models_{\mathfrak{A}_{i}}\left(c_{l} \neq c_{j}\right)$ is true when $l \neq j$ and $\left|\mathfrak{A}_{i}\right|=\left\{c_{\alpha}^{\mathfrak{A}_{i}} \mid \alpha \in \mathcal{I}\right\}$. Where $c_{\alpha}$ is the $\alpha^{\prime}$ th constant in an enumeration of the set of constants of $L$ and $\mathcal{I}$ is the index of enumeration. Note $C=\left\{c_{\alpha} \mid \alpha \in \mathcal{I}\right\}$

- If $\phi$ is a formula of $L$ and $\operatorname{ter}(\phi)-(C \cup V)=m_{i}$, then for all variable assignments $v$ for $\mathfrak{A}_{i}$ we have $\operatorname{Contx}(\phi, v)=\left(\models_{\mathfrak{A}_{i}} \phi[v]\right)$

We have the following for $C$ :

- For each $c \in C$ and each $i \in \kappa$ where $\left|\mathfrak{A}_{i}\right| \neq \varnothing$, we take a new constant $c^{i} \notin C$ where for $i, j \in \kappa$ and $a, b \in C$ we have $a^{i} \neq b^{j}$ if $i \neq j$ or $a \neq b$. We take $\mathfrak{C}=C \cup\left\{c^{i}|c \in C \wedge i \in \kappa \wedge| \mathfrak{A}_{i} \mid \neq \varnothing\right\}$ and for $i<\kappa, C_{i}=\left\{c^{i} \mid c \in C\right\}$. 
- We take $L_{i}$ to be a language where $C_{i} \subset L_{i}$ and $L_{i}-C_{i}=L-C$.

- For a formula $\phi$ of $L$ we will take $\phi^{i}$ to be the formula of $L_{i}$ given by substituting for each occurrence of a constant $c$ in $\phi$ the new constant $c^{i}$.

- We take $\mathfrak{A}_{i}^{*}$ to be the structure where if $v$ is a variable assignment for $\mathfrak{A}_{i}$ and $\phi$ is a formula of $L$, then $\left(\models_{\mathfrak{A}_{i}} \phi[v]\right)=\left(\models_{\mathfrak{A}_{i}^{*}}(\phi[c v])^{i}\right)$. Note that given the preceding is true we have:

* For all $i \in \kappa$ if $\left|\mathfrak{A}_{i}\right| \neq \varnothing$, then $\models_{\mathfrak{A}_{i}^{*}} c_{l}^{i} \neq c_{j}^{i}$ is true when $l \neq j$ and $\left|\mathfrak{A}_{i}^{*}\right|=\left\{\left(c_{\alpha}^{i}\right)^{\mathfrak{A}_{i}} \mid \alpha \in \mathcal{I}\right\}$

- We take $\Delta_{i}$ as given in the definition of Contx being a $\kappa$-simple contextual model. We let $\Delta_{i}^{\prime}=\left\{\psi^{i} \mid \psi \in \Delta_{i}\right\}$ and we note that since $\models_{\mathfrak{A}_{i}} \psi$ is true for all $\psi \in \Delta_{i}$ we have $\models_{\mathfrak{A}_{i}^{*}} \psi^{i}$ for all $\psi^{i} \in \Delta_{i}^{\prime}$

- We take

$$
\Delta_{i}^{\prime \prime}=\Delta_{i}^{\prime} \cup\left\{t=c^{i} \mid t \in \operatorname{Con}\left(m_{i}, \mathfrak{A}_{i}^{*}\right) \cup C_{i} \text { and } \models_{\mathfrak{A}_{i}^{*}}\left(t=c^{i}\right) \text { is true }\right\}
$$

And

$$
\Delta_{i}^{*}=\Delta_{i}^{\prime \prime} \cup\left\{\phi(t) \mid \exists\left\{\phi\left(t^{\prime}\right), t=c^{i}, t^{\prime}=c^{i}\right\} \subseteq \Delta_{i}^{\prime \prime}\right\} \cup\left(\bigcup_{l \neq m}\left\{c_{l}^{i} \neq c_{m}^{i}\right\}\right)
$$

- We let

$$
\Gamma_{1}=\left\{p\left(a_{1}, \ldots, a_{m}\right) \mid a_{1}, \ldots, a_{m} \in C \wedge p \in \mathcal{P} \wedge\left(\models_{\mathfrak{A}_{i}^{*}} p\left(a_{1}^{i}, \ldots, a_{m}^{i}\right) \text { is true }\right)\right\}
$$

and

$\Gamma_{2}=\left\{\neg p\left(a_{1}, \ldots, a_{m}\right) \mid a_{1}, \ldots, a_{m} \in C \wedge p \in \mathcal{P} \wedge\left(\models_{\mathfrak{A}_{i}^{*}} \neg p\left(a_{1}^{i}, \ldots, a_{m}^{i}\right)\right.\right.$ is true $\left.)\right\}$

where $\mathcal{P}$ denotes the set of predicate symbols of $L$ and $m$ ranges over the number of arguments for the predicates of $\mathcal{P}$. Let $\Gamma=\Gamma_{1} \cup \Gamma_{2}$

To attain our Anti-Structure we take:

$Y^{\prime}=\Gamma \cup\{c=c \mid c \in C\} \cup\left\{\left(\pi(a)=c \mid c \in C\right.\right.$ and $a=c$ or $a=c^{i}$ for $\left.i \in \kappa\right\} \cup \bigcup_{i \in \kappa} \Delta_{i}^{*}$

and show that $Y^{\prime}$ can be extended to a consistent system for the language $\mathfrak{L}=\pi L \cup \bigcup_{i \in \kappa} L_{i}$.

We now have the following for $Y^{\prime}$ :

- Every formula of $Y^{\prime}$ is a quantifier free sentence of $\mathfrak{L}$ (equivalently a variable free formula of $\mathfrak{L}$ ) 
- If $t$ is a terminal term of $\psi^{i} \in \Delta_{i}^{*}$, then $t$ is a variable free term of $L_{i}$ and there exists a constant $c^{i}$ of $L_{i}$ where $t=c^{i} \in Y^{\prime}$. Note that $t$ being a variable free term of $L_{i}$ and $\left|\mathfrak{A}_{i}^{*}\right|=\left\{\left(c_{\alpha}^{i}\right)^{\mathfrak{A}_{i}} \mid \alpha \in \mathcal{I}\right\}$ yields that there must exist a $c^{i}$ where $\models_{\mathfrak{A}_{i}^{*}}\left(t=c^{i}\right)$.

- We see that $Y^{\prime}$ is a consistent set of sentences as follows:

a. We have that if $\eta \in \Delta_{i}^{*}, \rho \in \Delta_{j}^{*}$ where $i \neq j$ and if $\sigma \in \Gamma \cup\{c=c \mid c \in$ $C\} \cup\left(\bigcup_{l \neq m}\left\{c_{l} \neq c_{m}\right\}\right)$, then $\eta, \rho$ and $\sigma$ are quantifier free sentences sharing no constant symbols. We also have the consistency of $\Delta_{i}^{*}$ implies the consistency of $\left.\Gamma \cup\{c=c \mid c \in C\} \cup\left(\bigcup_{l \neq m}\left\{c_{l} \neq c_{m}\right)\right\}\right)$ where it is easily seen that $\Delta_{i}^{*} \subseteq\left\{\psi \mid \psi\right.$ is a sentence and $\models_{\mathfrak{A}_{i}^{*}} \psi$ is true $\}$ for all $i \in \kappa$, yields that each $\Delta_{i}^{*}$ is a consistent set of quantifier free sentences. It follows from the preceding that

$$
\Gamma \cup\{c=c \mid c \in C\} \cup \bigcup_{i \in \kappa} \Delta_{i}^{*} \cup\{a \neq b \mid a, b \in \mathfrak{C}[a \neq b]\}
$$

is a consistent set of sentences. The proof of the preceding follows from the claim that given $S$ is a finite set of quantifier free sentence where each sentence in $S$ is satisfiable and no pair of sentences of $S$ share any constant symbols, then $S$ is satisfiable. More over we note that for the $S$ given in the preceding that $S$ is satisfiable by a structure $\mathfrak{B}$ where if $\psi_{1}, \psi_{2} \in S$ with $\psi_{1} \neq \psi_{2}$ and $a_{i}$ is a constant symbol of $\psi_{i}$, then $a_{1}^{\mathfrak{B}} \neq a_{2}^{\mathfrak{B}}$. To be more clear we are using the claim to attain that

$$
\Gamma \cup\{c=c \mid c \in C\} \cup \bigcup_{i \in \kappa} \Delta_{i}^{*} \cup\{a \neq b \mid a, b \in \mathfrak{C}[a \neq b]\}
$$

is finitely satisfiable and therefore by the compactness theorem for first order logic, is satisfiable. We also note that if $X_{1}, \ldots, X_{n}$ are finite sets of formulas each a subset of a different one of the sets $\left(\Gamma \cup\{c=c \mid c \in C\} \cup\left(\bigcup_{l \neq m}\left\{c_{l} \neq c_{m}\right\}\right)\right.$ or $\Delta_{i}^{*}$ for some $i \in \kappa$, then $\bigwedge X_{j}$ is a single formula and if $S=\left\{\bigwedge X_{1}, \ldots, \bigwedge X_{m}\right\}$, then each formula of $S$ would be a quantifier free sentence sharing no constant symbols with any other formula of $S$, and then $S$ is satisfiable if and only if $\{a \neq b \mid a, b \in \mathfrak{C}[a \neq b]\} \cup \bigcup_{j \leq m} X_{j}$ is satisfiable if and only if $\bigcup_{j \leq m} X_{j}$ is satisfiable.

b. Taking $\mathfrak{B}$ to be a structure for the language

$$
C \cup \bigcup_{i \in \kappa} L_{i}
$$


with $\mathfrak{B}$ a model of

$$
\Gamma \cup\{c=c \mid c \in C\} \cup \bigcup_{i \in \kappa} \Delta_{i}^{*} \cup\{a \neq b \mid a, b \in \mathfrak{C}[a \neq b]\}
$$

We note that we can add the unary function symbol $\pi$ to $C \cup \bigcup_{i \in \kappa} L_{i}$ and extend our structure $\mathfrak{B}$ to $\mathfrak{B}^{\prime}$ so that $\pi^{\mathfrak{B}^{\prime}}\left(c^{i}\right)^{\mathfrak{B}^{\prime}}=c^{\mathfrak{B}^{\prime}}$ to attain a structure for our extended language. We note that $\mathfrak{B}^{\prime}$ is a model of $Y^{\prime}$ and therefore $Y^{\prime}$ is consistent.

To attain our consistent system $Y$ for $\mathfrak{L}$ we give:

- We take

$\Psi_{1}=\left\{\psi \mid \psi\right.$ is a variable free formula of $\mathfrak{L}$ and $\left.\forall t \in \operatorname{ter}(\psi) \exists c \in \mathfrak{C}\left[t=c \in Y^{\prime}\right]\right\}$

and

$\Psi=\left\{\psi \mid \psi\right.$ is a variable free formula of $\mathfrak{L}-\{=\}$ and $\left.\forall t \in \operatorname{ter}(\psi) \exists c \in \mathfrak{C}\left[t=c \in Y^{\prime}\right]\right\}$

- We take $\Sigma_{1}$ to be equal to the consequences of $Y^{\prime}$.

- Given $\Sigma$ is a maximal set of consistent formulas in our language, where $\Sigma_{1} \subseteq \Sigma$, we take $Y$ to be the consequences of $(\Sigma \cap \Psi) \cup\left(\Sigma_{1} \cap \Psi_{1}\right)$ intersect $\Psi_{1}$.

To see that $Y$ is a system we note the following:

- Condition 1 for being a system is met by $Y$, since given $a \in \mathfrak{C}$ there exists $c \in C$ where either $a=c$ or $a=c^{i}$ and then we have either $c=c \in Y^{\prime}$ and $c^{i}=c^{i} \in \Delta^{*} \subset Y^{\prime}$.

- Condition 2 of our requirements for $Y$ being a system is meet because for all $\psi \in Y$ we clearly have $\psi \in Y \subseteq \Psi_{1}$ and therefore by definition for all $t \in \operatorname{ter}(\psi)$ there exists a $c \in \mathfrak{C}$ where $t=c \in Y^{\prime} \subseteq Y$

- Requirement 3 follows from the fact that $Y$ is a subset of $\Psi_{1}$ and $Y$ is closed under logical consequence in $\psi_{1}$.

- Requirements 4 for $Y$ being a system is fulfilled, since $(\Sigma \cap \Psi) \subseteq Y$ where $\Sigma$ is a maximal set of consistent formulas on our language and

$$
\left(\bigcup\left\{\left\{p\left(a_{0}, \ldots, a_{m}\right), \neg p\left(a_{0}, \ldots, a_{m}\right)\right\} \mid\left(p \in \mathcal{P} \text { and } a_{0}, \ldots, a_{m} \in \mathfrak{C}\right)\right\}\right) \subseteq \Psi
$$

- Requirement 5 is seen to be satisfied by our construction of each $\Delta_{i}^{*}$ and the comment dealing with $(Y \models(t=c)$ if and only if $(t=c) \in Y)$ found in the proof of Theorem 3.18. 
We take $\models_{\mathfrak{A}}^{A}$ to be the Anti-Structure giving the system $Y$, where from the proof of Theorem 3.18 we have $A=\mathfrak{C}^{\mathfrak{A}}$. We take for any finite set of terms $S$ of $\mathfrak{L}$ $\operatorname{dom}(S)=\left\{v \mid v\right.$ is a variable assignment and $\models_{\mathfrak{A}}^{A} \bigwedge_{t \in S}(t=t)[v]$ is true $\}$.

We note the following:

1. If given $\phi$ is a formula of $C \cup \bigcup_{i \in \kappa} L_{i}$ and $\psi_{\phi}$ is the formula where $\phi$ is given by replacing in $\psi_{\phi}$ each occurrence of $\pi(t)$ with $t$, for each $\pi t \in \operatorname{ter}(\psi)$ where $\operatorname{ter}(\phi) \cap \operatorname{ter}(\psi)=\varnothing$, then we have:

i. If $p$ is an $m$-ary predicate and $a_{1}, \ldots, a_{m} \in C$, then because $\Gamma \cup\left\{(\pi(a)=c) \mid c \in C\right.$ and $a=c$ or $a=c^{i}$ for $\left.i \in \kappa\right\} \subseteq Y$ we have for $b_{j} \in\left\{a_{j}\right\} \cup\left\{a_{j}^{l} \mid l \in \kappa\right\}$ that

$$
\left(\models_{\mathfrak{A}}^{A} p\left(a_{1}, \ldots, a_{m}\right)\right)=\left(\models_{\mathfrak{A}_{i}^{*}} p\left(a_{1}^{i}, \ldots, a_{m}^{i}\right)\right)=\left(\models_{\mathfrak{A}}^{A} p\left(\pi\left(b_{1}\right), \ldots, \pi\left(b_{m}\right)\right)\right)
$$

ii. From (i) above a simple proof by induction on the quantifier free sentences yields that if $\phi$ is a quantifier free formula of $L$ with $\operatorname{ter}(\phi)-$ $(C \cup V)=m_{i}$ and $v \in \operatorname{Var} A_{i}$, taking $\Phi$ to be $(\phi[c v])^{i}$ and $v^{i}(x)=$ $\left(c v(x)^{i}\right)^{\mathfrak{A}_{i}^{*}}$, we note that $\{\Phi, \neg \Phi\} \cap Y \neq \varnothing$ and therefore:

$$
\operatorname{Contx}(\phi, v)=\left(\models_{\mathfrak{A}_{i}} \phi[v]\right)=\left(\models_{\mathfrak{A}_{i}^{*}} \Phi\right)=\left(\models_{\mathfrak{A}}^{A}(\phi)^{i}\left[v^{i}\right]\right)=\left(\models_{\mathfrak{A}}^{A} \psi_{\phi}\left[v^{i}\right]\right)
$$

2. Taking $v z$ to be any variable assignment where for $c \in C$ we have $v z\left(z_{c}\right)=$ $c^{\mathfrak{A}}$ we have the following:

i. For a quantifier free formula $\phi$ of $L$ where $\operatorname{ter}(\phi)-(C \cup V)=m_{i}$ and a variable assignment $v \in \operatorname{Var} A_{i}$, if $(\phi[c v])^{\prime}$ is the formula given in the definition of $\pi(\phi[c v])$, then $\phi[c v]$ is the formula $(\phi[c v])^{\prime}[c v z]$ and if $v z^{i}$ is the variable assignment $v z^{i}\left(z_{c}\right)=\left(c^{i}\right)^{\mathfrak{A}_{i}^{*}}$, then taking $\psi_{\phi}$ as defined in (1) above and applying the result of 1 , we have

$$
\operatorname{Contx}(\phi, v)=\left(\models_{\mathfrak{A}}^{A} \psi_{(\phi[c v])^{\prime}}\left[v z^{i}\right]\right)
$$

- Noting that if $(\phi[c v])^{\prime \prime}$ is as given for the definition of $\pi(\phi[c v])$, then $\psi_{(\phi)^{\prime \prime}}$ is the formula

$$
\bigwedge_{c \in c n s(\phi[c v])}\left(\pi\left(z_{c}\right)=\pi(c)\right)
$$

and therefore $\models_{\mathfrak{A}}^{A} \psi_{(\phi)^{\prime \prime}}\left[v z^{i}\right]$ is always true.

It follows from $\mathrm{i}$ and ii above, that if $\operatorname{Contx}(\phi, v)$ is true, then $\models_{\mathfrak{A}}^{A} \psi_{(\phi)^{\prime \prime}} \wedge \psi_{(\phi)^{\prime}}\left[v z^{i}\right]$ is true. Noting that $\pi(\phi[c v])$ is the formula $\exists z_{c_{0}}, \ldots, \exists z_{c_{n}}\left(\psi_{(\phi)^{\prime \prime}} \wedge \psi_{(\phi)^{\prime}}\right)$, we have that if $\operatorname{Contx}(\phi, v)$ is true, then $\models_{\mathfrak{A}}^{A}$ $\pi(\phi[c v])$ is true. 
3. Given $\phi$ is any quantifier free formula and $v$ is any variable assignment for $\models_{\mathfrak{A}}^{A}$, then it follows from $1 \mathrm{i}$ above that if $\eta$ is attained from $\phi$ by replacing any constant symbol $c^{i}$ ( for $c \in C$ with $c^{0}=c$ ) which is an argument for a predicate of $\phi$ with the constant symbol $c^{j}$ (note for the same $c \in C$ which gives $\left.c^{i}\right)$, then $\left(\models_{\mathfrak{A}}^{A} \psi_{\phi}[v]\right)=\left(\models_{\mathfrak{A}}^{A} \psi_{\eta}[v]\right)$.

It follows given $\phi$ is a formula of $L$ where $\operatorname{ter}(\phi)-(C \cup V)=m_{i}$, and $v \in \operatorname{Var} A_{i}$, taking $(\phi[c v])^{\prime \prime}$ and $(\phi[c v])^{\prime}$ to be the formulas given in the definition of $\pi(\phi[c v])$, if $\forall s \in \operatorname{dom}\left(m_{i}\right)$ and $\forall t \in \operatorname{ter}(\phi)-(C \cup V)$, we have $(\pi t)^{\mathfrak{A}}\left[s^{i}\right]=(\pi t)^{\mathfrak{A}}[s]$, then:

i. We assume $s$ is a variable assignment where $\models_{\mathfrak{A}}^{A} \psi_{\left((\phi[c v])^{\prime \prime} \wedge(\phi)^{\prime}\right)}[s]$ is true. We note:

ii. Given $\models_{\mathfrak{A}}^{A} \psi_{\left((\phi[c v])^{\prime \prime} \wedge(\phi)^{\prime}\right)}[s]$ is true, by definition we then have $\models_{\mathfrak{A}}^{A}$ $\psi_{(\phi[c v])^{\prime}}[s]$ is true.

iii. From our assumptions on $\phi$ we have that if $t\left(a_{1}, \ldots, a_{m}\right) \in \operatorname{ter}(\phi[c v])-$ $C$ for constants $a_{1}, \ldots, a_{m} \in C$, then

$$
\left(\pi t\left(z_{a_{1}}, \ldots, z_{a_{m}}\right)\right)^{\mathfrak{A}}\left[s^{i}\right]=\left(\pi t\left(z_{a_{1}}, \ldots, z_{a_{m}}\right)\right)^{\mathfrak{A}}[s]
$$

iv. Taking $\eta$ to be the formula that is attained from $(\phi[c v])^{\prime}$ by replacing each terminal occurrence of a variable $z_{a}$ with the constant $c \in \mathfrak{C}$ where $s\left(z_{a}\right)=c^{\mathfrak{A}}$ and leaving all other occurrences of variables the same, it follows from iii that $\left(\models_{\mathfrak{A}}^{A} \psi_{(\phi[c v])^{\prime}}[s]\right)=\left(\models_{\mathfrak{A}}^{A} \psi_{\eta}\left[s^{i}\right]\right)$.

v. From the opening part of 3 above we have that if $\rho$ is the formula attained by replacing each terminal constant symbol $c^{j}$ (where $c \in C$ and $j$ is allowed to equal 0 with $\left.c^{0}=c\right)$ of $\eta$ with $c^{i}$, then $\left(\models_{\mathfrak{A}}^{A}\right.$ $\left.\psi_{\rho}\left[s^{i}\right]\right)=\left(\models_{\mathfrak{A}}^{A} \psi_{\eta}\left[s^{i}\right]\right)$. We note that $\left(\models_{\mathfrak{A}}^{A} \psi_{(\phi[c v])^{\prime}}\left[s^{i}\right]\right)=\left(\models_{\mathfrak{A}}^{A} \psi_{\rho}\left[s^{i}\right]\right)$ and therefore $\models_{\mathfrak{A}}^{A} \psi_{(\phi[c v])^{\prime}}[s]$ is true, gives $\models_{\mathfrak{A}}^{A} \psi_{\eta}\left[s^{i}\right]$ is true, gives $\models_{\mathfrak{A}}^{A} \psi_{\rho}\left[s^{i}\right]$ is true, gives $\models_{\mathfrak{A}}^{A} \psi_{(\phi[c v])^{\prime}}\left[s^{i}\right]$ is true.

vi. From (i-v) above having $\models_{\mathfrak{A}}^{A} \pi(\phi[c v])$ is true yields there exist a variable assignment $s$ where $\models_{\mathfrak{A}}^{A} \psi_{\left((\phi[c v])^{\prime \prime} \wedge(\phi)^{\prime}\right)}[s]$ is true, which yields $\models_{\mathfrak{A}}^{A} \psi_{(\phi[c v])^{\prime}}[s]$ is true and consequently $\models_{\mathfrak{A}}^{A} \psi_{(\phi[c v])^{\prime}}\left[s^{i}\right]$ is true. From 1 above we have $\models_{\mathfrak{A}}^{A} \psi_{(\phi[c v])^{\prime}}\left[s^{i}\right]$ is true, gives $\models_{\mathfrak{A}}^{A}\left((\phi[c v])^{\prime}\right)^{i}\left[s^{i}\right]$ is true where $(\phi[c v])^{\prime}$ being constant free yields it is the same formula as $\left((\phi[c v])^{\prime}\right)^{i}$ and therefore we have $\models_{\mathfrak{A}}^{A}(\phi[c v])^{\prime}\left[s^{i}\right]$ is true. We now have $\models_{\mathfrak{A}}^{A} \pi(\phi[c v])$ is true, gives $\models_{\mathfrak{A}}^{A}\left((\phi[c v])^{\prime}\right)\left[s^{i}\right]$ is true, where noting $s^{i}\left(z_{c}\right)=\left(c^{i}\right)^{\mathfrak{A}}$ we then have

$$
\left(\models_{\mathfrak{A}}^{A}(\phi[c v])^{\prime}\left[s^{i}\right]\right) \Rightarrow\left(\models_{\mathfrak{A}}^{A}(\phi[c v])^{i}\right) \Rightarrow\left(\models_{\mathfrak{A}_{i}} \phi[v]\right) \Rightarrow \operatorname{Contx}(\phi, v)
$$

and therefore under our assumptions for $\phi$ and $m_{i}$, we have $\models_{\mathfrak{A}}^{A} \pi(\phi[c v])$ is true, gives there exist a variable assignment $s$ where $\models_{\mathfrak{A}}^{A}\left((\phi[c v])^{\prime}\right)^{i}\left[s^{i}\right]$ is true, which gives $\operatorname{Contx}(\phi, v)$ is true. 
From 1,2 , and 3 directly above, we have that if $\forall i \in \kappa \forall s \in \operatorname{dom}\left(m_{i}\right), \forall t \in$ $m_{i}$, it is the case that $(\pi t)^{\mathfrak{A}}\left[s^{i}\right]=(\pi t)^{\mathfrak{A}}[s]$, then for all $v \in \operatorname{Var} A_{i}$ and all quantifier free formulas $\phi$ where $\operatorname{ter}(\phi)-(C \cup V)=m_{i}$, we have $\operatorname{Contx}(\phi, v)=\left(\models_{\mathfrak{A}}^{A}\right.$ $\pi(\phi[c v])$.

We complete this proof by showing $\forall i \in \kappa \forall s \in \operatorname{dom}\left(m_{i}\right), \forall t \in m_{i}$, it is the case that $(\pi t)^{\mathfrak{A}}\left[s^{i}\right]=(\pi t)^{\mathfrak{A}}[s]$.

To these ends we give the following:

$b_{1}$. Given $t\left(x_{1}, \ldots, x_{n}\right)$ is a constant free term of $L, r$ is a variable free term of $\mathfrak{L}$ and $a_{1}, \ldots, a_{n} \in \mathfrak{C}$, then from our construction of $Y$, we have $t\left(a_{1}, \ldots, a_{n}\right)=$ $r \in Y$ if and only if there exists $b_{1}, b_{2} \in \mathfrak{C}$, where $\left\{t\left(a_{1}, \ldots, a_{n}\right)=b_{1}, r=\right.$ $\left.b_{2}\right\} \subseteq Y^{\prime}$ and $\left(b_{1}=b_{2}\right) \in Y$. It then follows that $b_{1}$ and $b_{2}$ must be the same constant symbol and that there must exist an $i<\kappa$ where $\left\{t\left(a_{1}, \ldots, a_{n}\right)=b_{1}, r=b_{1}, t\left(a_{1}, \ldots, a_{n}\right)=r\right\} \subseteq \Delta_{i}^{*}$. We now note that we also have:

$$
I^{*} . t\left(a_{1}, \ldots, a_{n}\right) \in \operatorname{Con}\left(m_{i}, \mathfrak{A}_{i}^{*}\right)
$$

$b_{2}$. For a constant free term $t$ of $L$, it follows from the preceding and the fact $Y$ is the system for $\models_{\mathfrak{A}}^{A}$, that if for all $i \in \kappa,\left\{a_{1}, \ldots, a_{n}\right\}-C_{i} \neq \varnothing$, then $\models_{\mathfrak{A}}^{A} t\left(a_{1}, \ldots, a_{n}\right)=t\left(a_{1}, \ldots, a_{n}\right)$ is undefined.

$b_{4}$. From the construction of $Y, \pi\left(t\left(a_{1}, \ldots, a_{n}\right)\right)=c \in Y$ if and only if there exists an $i<\kappa$ where $t\left(a_{1}, \ldots, a_{n}\right)=c^{i} \in Y^{\prime}$.

$b_{5}$. For $\mathfrak{c} s \in \mathfrak{C} V a r$ we will take $\mathfrak{c} s^{i} \in \mathfrak{C} V$ ar to be the variable to constant assignment where $\mathfrak{c} s^{i}(x)=c^{i}$ if and only if $\mathfrak{c} s(x)=c^{j}$ for any $j<\kappa$ and any given $c \in C$ ( We will allow $j=0$ and have $c^{0}=c$ ). We will take $c s \in C V a r$ so that for all variables $x$ there exists an $l_{x}<\kappa$ where $c s(x)^{l_{x}}=\mathfrak{c} s$.

$b_{6}$. We note that if our condition $v$ of the definition of a simple contextual model holds, then the condition must also hold when we replace $C$ with $C_{i}$ in the antecedent, and both $c v$ with $c v^{i}$, and $\mathfrak{A}_{i}$ with $\mathfrak{A}_{i}^{*}, \mathfrak{A}_{j}$ with $\mathfrak{A}_{j}^{*}$ throughout the statement. To show that for $t \in m_{i}$ and $s \in \operatorname{dom}\left(m_{i}\right)$ we have $\models_{\mathfrak{A}}^{A} \pi t[s]=\pi t\left[s^{i}\right]$ is true (equivalently $\pi t^{\mathfrak{A}}[s]=\pi t^{\mathfrak{A}}\left[s^{i}\right]$ under our assumptions on $t$ and $s$ ), we give a proof by contradiction. To these ends we suppose $t \in m_{i}, s \in \operatorname{dom}\left(m_{i}\right)$ and $\models_{\mathfrak{A}}^{A} \pi t[\mathfrak{c} s] \neq \pi t\left[\mathfrak{c} s^{i}\right]$ is true. It follows:

a. From the above we have $c_{1}, c_{2} \in C$ and $j<\kappa$ where $c_{1} \neq c_{2}, i \neq j$ and $t[\mathfrak{c} s]=c_{1}^{j} \in \Delta_{j}^{*}$ while $t\left[\mathfrak{c} s^{i}\right]=c_{2}^{i} \in \Delta_{i}^{*}$. Note $i=j$ would yield $t[\mathfrak{c s}]$ is $t\left[\mathfrak{c} s^{i}\right]$ and we would immediately be in contradiction with our assumptions. Note that we also have that if $x$ is a variable occurring in $t$, then $\mathfrak{c} s(x) \in C_{j}$ and therefore $t[\mathfrak{c} s]$ is the exact same term as $t\left[\mathfrak{c} s^{j}\right]$. 
b. It follows from $b_{1}$ that $I^{*}$ holds for $t[\mathfrak{c} s]$ with regard to $m_{j}$, and that $t[\mathfrak{c} s] \in m_{i}[\mathfrak{c} s]$. From $a$ above and the preceding statement we have $t\left[\mathfrak{c} s^{j}\right] \in m_{i}\left[\mathfrak{c} s^{j}\right] \cap \operatorname{Con}\left(m_{j}, \mathfrak{A}_{j}^{*}\right)$ and consequently we are meeting the conditions for requirement $v$ of the definition of a $\kappa$-simple contextual model.

c. It follows we have both $t\left[c s^{i}\right]^{\mathfrak{R}_{i}^{*}}=\left(c_{2}^{i}\right)^{\mathfrak{A l}_{i}^{*}}$ and $t\left[c s^{j}\right]^{\mathfrak{A}_{j}^{*}}=\left(c_{1}^{j}\right)^{\mathfrak{A}_{j}^{*}}$, so that $t[c s]^{\mathfrak{A}_{i}}=\left(c_{2}\right)^{\mathfrak{A L}_{i}}$ and $t[c s]^{\mathfrak{A}_{j}}=\left(c_{1}\right)^{\mathfrak{A}_{j}}$. It is clear that the consequence of the conditional statement $v$ in the definition of a $\kappa$ simple contextual model does not hold and therefore the negation of its antecedent must hold to avoid a contradiction.

d. Since the negation of the antecedent of $v$ holds we must have an $r[c s] \in m_{i}[c s]$ which satisfies the conditions $a_{1}$ to $a_{3}$ for requirement $v$, where then because $s \in \operatorname{dom}\left(m_{i}\right)$ we have from the above that there exists a $c \in C$ and an $l<\kappa$ where $r_{i}[\mathrm{c} s]=c^{l} \in \Delta_{l}^{*}$.

e. Since $b_{2}$ above gives that $r[\mathfrak{c} s]=c^{l} \in \Delta_{l}^{*}$ and $t[\mathfrak{c} s]=c_{1}^{j} \in \Delta_{j}^{*}$ yields all constants occurring in $r[\mathfrak{c} s]$ and $t[\mathfrak{c} s]$ must be subsets of $C_{l}$ and $C_{j}$ respectively and since part $a_{2}$ of requirement $v$ gives that either $t[\mathfrak{c} s]$ is a sub-term of $r[\mathfrak{c} s]$ or $r[\mathfrak{c} s]$ is a sub-term of $t[\mathfrak{c} s]$, we must have $l=j$. Note that we then have $r[\mathfrak{c s}]$ is the term $r\left[\mathfrak{c} s^{j}\right]$.

f. From $e$ above we must have that $I *$ holds for $r[\mathfrak{c} s]$ ( which is the term $\left.r\left[\mathfrak{c} s^{j}\right]\right)$, so that $r\left[\mathfrak{c} s^{j}\right] \in \operatorname{Con}\left(m_{j}, \mathfrak{A}_{j}^{*}\right)$. We now have a contradiction since we must also have $a_{2}$ holds for $r\left[\mathfrak{c} s^{j}\right]$ where $a_{1}$ gives that $r\left[\mathfrak{c} s^{j}\right] \notin$ $\operatorname{Con}\left(m_{j}, \mathfrak{A}_{j}^{*}\right)$.

As we have shown:

- $\forall i \in \kappa \forall s \in \operatorname{dom}\left(m_{i}\right), \forall t \in m_{i}$, it is the case that $(\pi t)^{\mathfrak{A}}\left[s^{i}\right]=(\pi t)^{\mathfrak{A}}[s]$,

- and we have shown that if $\forall i \in \kappa \forall s \in \operatorname{dom}\left(m_{i}\right), \forall t \in m_{i}$, it is the case that $(\pi t)^{\mathfrak{A}}\left[s^{i}\right]=(\pi t)^{\mathfrak{A}}[s]$, then for all $i \in \kappa$ and for all $\phi$ where $\operatorname{ter}(\phi)-(C \cup V)=m_{i}$ and all variable assignments $v \in \operatorname{Var} A_{i}$, we have $\operatorname{Contx}(\phi, v)=\models_{\mathfrak{A}}^{A} \pi(\phi[c v])$

we have completed our proof of the First Model Completeness Theorem.

Definition 3.26. If $\models_{\mathfrak{A}}^{A}$ is an Anti-Structure witnessing the validity of the First Model Completeness Theorem for a simple Contextual model Contx of a language $L$, then we will call $\models_{\mathfrak{A}}^{A}$ an Anti-Structure model of the Simple Contextual Model, and refer to it as a simple contextual Anti-Structure model. When an experiment has a Simple Contextual Model, then we will call an Anti-Structure model of the Simple Contextual model for the experiment an Anti-Structure model for the experiment.

Corollary 3.27. If an experiment has a Simple Contextual model, then an experiment has an Anti-Structure model. 
In the orthohelium example of [5], Kochen and Specker prove that assigning values for the measurement of the orthohelium for the different measurements corresponding to collections of orthonormal basis leads to the contradiction of having a direction of measurement being multi-valued. That is, there exists two collections of orthonormal bases which share a common vector, where that vector must give different measured values of the orthohelium for the two cases. We build an Anti-Structure model for orthohelium that resolves this contradiction with an Anti-Structure that gives value to functions representing in their action the components of angular momentum of orthohelium, and thus yields a meaningfully representation of the experiment with regard to the issue at hand.

We apply Theorem 3.25 to give our model. The model will give the overall angular momentum of the orthohelium as the term $h\left(c_{0}\right)$ where $c_{0}$ is a constant representing the origin, and a terminal term with argument $c_{0}$ represents an action of measurement of the orthohelium as it is perturbed from the origin $c_{0}$ (see [5] for use of perturbed). For a vector $\mathbf{v}$ from an orthonormal basis we will have the term $f_{\mathbf{v}}\left(c_{0}\right)$ represent the measurement of the orthohelium from the direction $\mathbf{v}$. We will then have that a measurement of the angular momentum of orthohelium from orthogonal unit vector directions $\mathbf{v}_{1}, \mathbf{v}_{2}$ and $\mathbf{v}_{3}$ is given by determining the truth value of a formula with terminal terms $h\left(c_{0}\right), f_{\mathbf{v}_{1}}\left(c_{0}\right)$, $f_{\mathbf{v}_{2}}\left(c_{0}\right)$ and $f_{\mathbf{v}_{3}}\left(c_{0}\right)$. Our set up will in accordance with theorem 3.25 yield an Anti-Structure model that satisfies the requirements given in Kochen and Speckers for their orthohelium example see [5].

Example 3.28. Let $\mathcal{V}$ be a collection of ordered orthonormal basis for $\mathbb{R}^{3}$ where for every orthonormal basis $\left\{\mathbf{x}_{1}, \mathbf{x}_{2}, \mathbf{x}_{3}\right\}$ of $\mathbb{R}^{3}$, there exists $\left\langle\mathbf{v}_{1}, \mathbf{v}_{2}, \mathbf{v}_{3}\right\rangle \in \mathcal{V}$ such that $\left\{\mathbf{x}_{1}, \mathbf{x}_{2}, \mathbf{x}_{3}\right\}=\left\{\mathbf{v}_{1}, \mathbf{v}_{2}, \mathbf{v}_{3}\right\}$, and if $\left\langle\mathbf{v}_{1}, \mathbf{v}_{2}, \mathbf{v}_{3}\right\rangle,\left\langle\mathbf{x}_{1}, \mathbf{x}_{2}, \mathbf{x}_{3}\right\rangle \in \mathcal{V}$ with $\left\langle\mathbf{v}_{1}, \mathbf{v}_{2}, \mathbf{v}_{3}\right\rangle \neq\left\langle\mathbf{x}_{1}, \mathbf{x}_{2}, \mathbf{x}_{3}\right\rangle$, then $\left\{\mathbf{v}_{1}, \mathbf{v}_{2}, \mathbf{v}_{3}\right\} \neq\left\{\mathbf{x}_{1}, \mathbf{x}_{2}, \mathbf{x}_{3}\right\}$. We will then have:

- $L$ be a first order language that contains the unary function symbols $h, f_{\mathbf{v}}$ (for $\|\mathbf{v}\|=1$ ), the binary function symbol + , and for each $\mathbf{v} \in \mathbb{R}^{3}$ a constant symbol $c_{\mathbf{v}}$.

- For every $\left\langle\mathbf{v}_{1}, \mathbf{v}_{2}, \mathbf{v}_{3}\right\rangle \in \mathcal{V}$, define

$$
M_{\left\langle\mathbf{v}_{1}, \mathbf{v}_{2}, \mathbf{v}_{3}\right\rangle}=\left\{h(s), f_{\mathbf{v}_{1}}(s), f_{\mathbf{v}_{2}}(s), f_{\mathbf{v}_{3}}(s),\left(f_{\mathbf{v}_{1}}(s)+f_{\mathbf{v}_{2}}(s)\right)+f_{\mathbf{v}_{3}}(s)\right\}
$$

- For each $\left\langle\mathbf{v}_{1}, \mathbf{v}_{2}, \mathbf{v}_{3}\right\rangle \in \mathcal{V}$, we define the structure $\mathfrak{A}_{\left\langle\mathbf{v}_{1}, \mathbf{v}_{2}, \mathbf{v}_{3}\right\rangle}$, with point set $\left|\mathfrak{A}_{\left\langle\mathbf{v}_{1}, \mathbf{v}_{2}, \mathbf{v}_{3}\right\rangle}\right|=\mathbb{R}^{3}$

- We will have $+{ }^{\left.\mathfrak{A}_{\left\langle\mathbf{v}_{1}, \mathbf{v}_{2}, \mathbf{v}_{3}\right\rangle}\right\rangle}$ is the usual + on $\mathbb{R}^{3}$.

- For each $v \in \mathbb{R}^{3}$ we will have $c_{v}^{\mathfrak{A}_{\left\langle\mathbf{v}_{1}, \mathbf{v}_{2}, \mathbf{v}_{3}\right\rangle}}=v$

- $f_{\mathbf{v}_{i}}^{\mathfrak{A}_{\left\langle\mathbf{v}_{1}, \mathbf{v}_{2}, \mathbf{v}_{3}\right\rangle}}$ is the constant function $\mathbf{v}_{i}$, for $i \in\{1,2\}, f_{\mathbf{v}_{3}}^{\mathfrak{A}_{\left\langle\mathbf{v}_{1}, \mathbf{v}_{2}, \mathbf{v}_{3}\right\rangle}}$ is the constant function $\mathbf{0}$, and $h^{\mathfrak{A}\left\{\mathbf{v}_{1}, \mathbf{v}_{2}, \mathbf{v}_{3}\right\rangle}$ is the constant function given by the sum of $f_{\mathbf{v}_{i}}^{\mathfrak{A}_{\left\langle\mathbf{v}_{1}, \mathbf{v}_{2}, \mathbf{v}_{3}\right\rangle}}$, for $i \in\{1,2,3\}$. 
- We now take $\phi_{\left\langle\mathbf{v}_{1}, \mathbf{v}_{2}, \mathbf{v}_{3}\right\rangle}$ to be the sentence

$$
\begin{aligned}
& \left(\left(h\left(c_{\mathbf{0}}\right)=\left(f_{\mathbf{v}_{1}}\left(c_{\mathbf{0}}\right)+f_{\mathbf{v}_{2}}\left(c_{\mathbf{0}}\right)\right)+f_{\mathbf{v}_{3}}\left(c_{\mathbf{0}}\right)\right) \wedge\left(f_{\mathbf{v}_{1}}\left(c_{\mathbf{0}}\right)=c_{\mathbf{v}_{1}}\right) \wedge\left(f_{\mathbf{v}_{2}}\left(c_{\mathbf{0}}\right)=c_{\mathbf{v}_{2}}\right) \wedge\left(f_{\mathbf{v}_{3}}\left(c_{\mathbf{0}}\right)=c_{\mathbf{0}}\right)\right) \\
& \text { so that }\left(\models_{\mathfrak{A}_{\left\langle\mathbf{v}_{1}, \mathbf{v}_{2}, \mathbf{v}_{3}\right\rangle}} \phi_{\left\langle\mathbf{v}_{1}, \mathbf{v}_{2}, \mathbf{v}_{3}\right\rangle}\right)=T .
\end{aligned}
$$

Note, that because we have $s$ is a subterm of all of our terms, and each $M_{\left\langle\mathbf{v}_{1}, \mathbf{v}_{2}, \mathbf{v}_{3}\right\rangle}$ is unique by a function symbol, the above gives a simple contextual model, and thus Theorem 3.25 gives an Anti-Structure $\models_{\mathfrak{A}}^{A}$, where

$$
\left(\models \models_{\mathfrak{A}}^{A} \pi\left(\phi_{\left\langle\mathbf{v}_{1}, \mathbf{v}_{2}, \mathbf{v}_{3}\right\rangle}\right)\right)=T
$$

for all $\left\langle\mathbf{v}_{1}, \mathbf{v}_{2}, \mathbf{v}_{3}\right\rangle \in \mathcal{V}$.

We now give the following for our next corollary.

Definition 3.29. If Contx gives a $\kappa$-simple contextual model where whenever $\operatorname{ter}(\phi)-(C \cup V)=m_{i}$, we have $\operatorname{Contx}(\phi, v)=\left(\models_{\mathfrak{A}_{i}} \phi[v]\right)$ for all variable assignment $v$ into $\left|\mathfrak{A}_{i}\right|$, then we define the complete simple contextual model CContx to be the extension of Contx to formulas $\psi$ which arise from the addition of quantification to formulas $\phi$, where $\operatorname{ter}(\phi)-(C \cup V)=m_{i}$ for some $i$, so that $\operatorname{CContx}(\psi, v)=\left(\models_{\mathfrak{A}_{i}} \psi[v]\right)$ for all variable assignments $v$ into $\left|\mathfrak{A}_{i}\right|$.

Corollary 3.30. If Contx is a $\kappa$-simple contextual model and $\models_{\mathfrak{A}}^{A}$ is the AntiStructure witness to Theorem 3.25 applied to Contx, then $\operatorname{CContx}(\psi, v)=\left(\models_{\mathfrak{A}}^{A} \pi(\psi[c v])\right)$.

Proof. Proof of Corollary 3.30

Given $x_{1}, \ldots, x_{n}$ are the variables which are quantified in $\psi$, we define $c v^{x_{1}, \ldots, x_{n}}$ so that $c v^{x_{1}, \ldots, x_{n}}\left(x_{l}\right)=x_{l}$ and for all variables $y \notin\left\{x_{1}, \ldots, x_{n}\right\}$ we will have $c v^{x_{1}, \ldots, x_{n}}(y)=c v(y)$. Given $\phi$ is $\psi$ without quantification and $\operatorname{ter}(\phi)-(C \cup V)=$ $m_{i}$, we will treat each $x_{l}$ for $l \leq n$, as if it is a constant and apply the same process used to give $\pi(\phi[c v])$ to attain $\pi\left(\phi\left[c v^{x_{1}, \ldots, x_{n}}\right]\right)$ ( assuming wlog that we now have variables $z_{x_{l}} \notin\left\{x_{1}, \ldots, x_{n}\right\}$ for all $\left.l \leq n\right)$. We note that $x_{1}, \ldots, x_{n}$ are now free in $\pi\left(\phi\left[c v^{x_{1}, \ldots, x_{n}}\right]\right)$ and more specifically only occur free in $\left(\phi\left[c v^{x_{1}, \ldots, x_{n}}\right]\right)^{\prime \prime}$ and only as the argument of $\pi$, when they occur in $\pi\left(\phi\left[c v^{x_{1}, \ldots, x_{n}}\right]\right)$. Taking $s$ to be a variable assignment for $\models_{\mathfrak{A}}^{A}$, we note that if $v^{\prime} \in \operatorname{Var} A_{i}$ so that $c v^{\prime}$ is the variable to constant assignment where $c v^{\prime}(y)=c v(y)$ for all $y \notin\left\{x_{1}, \ldots, x_{n}\right\}$ and $c v^{\prime}\left(x_{l}\right)=\left(\mathfrak{c} s\left(x_{l}\right)^{0}\right)$, then $\left(\models_{\mathfrak{A}}^{A} \pi\left(\phi\left[c v^{x_{1}, \ldots, x_{n}}\right][s]\right)=\operatorname{Contx}\left(\phi, v^{\prime}\right)=\left(\models_{\mathfrak{A}_{i}} \phi\left[v^{\prime}\right]\right)\right.$ and it is easily checked that from the definition of $\models_{\mathfrak{A}_{i}} \psi[v]$, we will have $\left(\models_{\mathfrak{A}}^{A} \pi(\psi[c v])\right)=\left(\models_{\mathfrak{A}_{i}} \psi[c v]\right)=\operatorname{CContx}(\psi, v)$.

Remark 3.31. From the proof of Theorem 3.25, it is seen that condition $v$, in the definition of a simple contextual model, was used to guarantee that if $\operatorname{Contx}(\phi, v)=F$, then $\models_{\mathfrak{A}}^{A} \pi(\phi[c v])=F$ when $\models_{\mathfrak{A}}^{A}$ is the Anti-Structure for Contx asserted by Theorem 3.25. An example of a contextual model which would fail to have a simple contextual model follows from taking $m_{1}=\{f(x)\}$, $m_{2}=\{f f(x)\}$ and structures $\mathfrak{A}_{1}, \mathfrak{A}_{2}$ where $\left|\mathfrak{A}_{i}\right|=\{0,1\}$ and for all $x, f^{\mathfrak{A}_{1}}(x)=$ 
0 while $f^{\mathfrak{A}_{2}}(x)=1$. We see that $f f(0) \in \operatorname{Con}\left(m_{1}, \mathfrak{A}_{1}\right)$ where $\models_{\mathfrak{A}_{1}} f f(0)=0$ while $\models_{\mathfrak{A}_{2}} f f(0)=1$. And we have that if $\models_{\mathfrak{A}}^{A}$ is the Anti-Structure generated for this contextual model using the process for generating the Anti-Structure witness to Theorem 3.25, then $\models_{\mathfrak{A}}^{A} \pi(f f(0)=0)$ would be true and disagree with the contextual model. From this example we see that condition $v$ failed because a second value for $f f(0)$ was defined, which confused the $\pi$ interpretation with regard to the formula $f f(0)=0$. As the confusion was derived from an errant second possibility for $f f(0)$, we may consider condition $v$ as preventing the the issue of having unintended values for terms. As such we can think of satisfaction of condition $v$ as preventing noise. In the proceeding example we will show how we can start with a contextual model having nice properties but fails condition $v$, and then make a simple adjustment, which we will think of as removing noise, to attain the satisfaction of condition $v$.

We have to this point avoided an analogous definition of quantum entanglement for Anti-Structures, but now that we have a notion of $\kappa$-simple contextual models which can have corresponding real world experimental counterparts, it is reasonable to give a definition of entanglement for a $\kappa$ - simple contextual Anti-Structure model. To these ends we have the following definition:

Definition 3.32. Given we have a simple contextual model with term set $m_{\alpha}$ for $\alpha<\kappa$, two variable free terms $t_{1}$ and $t_{2}$ will be simple contextual entangled in the simple contextual Anti-Structure Model $\models_{\mathfrak{A}}^{A}$ iff $\exists \alpha_{1}, \alpha_{2}<\kappa, \exists c v_{1}, c v_{2} \in$ $C V$ ar and $c_{i, j}$ for $i, j \in\{1,2\}$ where:

- $c_{1, j} \neq c_{2, j}$ when $j \in\{1,2\}$

- $\left\{t_{1}, t_{2}\right\} \subseteq M_{\alpha_{i}}\left[c v_{i}\right]$ for $i \in\{1,2\}$

- $\models_{\mathfrak{A}}^{A} \pi\left(\left(t_{1}=c_{i, 1} \wedge t_{2}=c_{i, 2} \wedge \bigwedge_{t \in M_{\alpha_{i}}} t=t\right)\left[c v_{i}\right]\right)$ is true for $i \in\{1,2\}$.

The following example is meant to demonstrate a process of removing noise from a contextual model to attain a simple contextual model, as well as give the behavior of a pair of non-trivial simple contextual entangled terms. The example will also show the utility of Theorem 3.25. In the example we have a loose interpretation of a real world pair of particles $p_{1}$ and $p_{2}$ which transition in state, where an examination of the final state of one or the other of the two particles produces a change in the state of both particles at a prior stage of transition. Ideally we would like to capture this as having functions $f_{1}$ and $g_{1}$ representing the transitions in state for our $p_{1}$ and $p_{2}$ respectively, and then for some initial condition $c_{0}$ have both $\left(f_{1}\left(c_{0}\right)=0\right) \wedge\left(g_{1}\left(c_{0}\right)=1\right) \wedge\left(f_{1} f_{1}\left(c_{0}\right)=\right.$ $\left.f_{1} f_{1}\left(c_{0}\right)\right)$ and $\left(f_{1}\left(c_{0}\right)=1\right) \wedge\left(g_{1}\left(c_{0}\right)=0\right) \wedge\left(g_{1} g_{1}\left(c_{0}\right)=g_{1} g_{1}\left(c_{0}\right)\right)$ be true. However it should be clear from Remark 3.31 that we might have to do some adjustments to a contextual model in order to get a $\kappa$-Simple contextual model of the entanglement. To these ends we give the following example:

Example 3.33. We start by giving the desired contextual model. We take: 
- $m_{1}=\left\{f_{1}(x), g_{1}(x), f_{1} f_{1}(x)\right\}$ and $m_{2}=\left\{f_{1}(x), g_{1}(x), g_{1} g_{1}(x)\right\}$

- We take $\left|\mathfrak{A}_{1}\right|=\left|\mathfrak{A}_{2}\right|=\{0,1\}$

- We will have $c_{0}^{\mathfrak{A}_{i}}=0$ and $c_{1}^{\mathfrak{A}_{i}}=1$

- For all $x$ we will have $f_{1}^{\mathfrak{A}_{1}}(x)=0, g_{1}^{\mathfrak{A}_{1}}(x)=1, f_{1}^{\mathfrak{A}_{1}}(x)=1, g_{1}^{\mathfrak{A}_{1}}(x)=0$.

It should be clear that the above gives a contextual model where $c_{0} \neq c_{1}$ and

- The truth of $\left(f_{1}\left(c_{0}\right)=c_{0}\right) \wedge\left(g_{1}\left(c_{0}\right)=c_{1}\right) \wedge\left(f_{1} f_{1}\left(c_{0}\right)=f_{1} f_{1}\left(c_{0}\right)\right)$ is given by $\models_{\mathfrak{A}_{1}}$ and is therefore true.

- While the truth of $\left(f_{1}\left(c_{0}\right)=c_{1}\right) \wedge\left(g_{1}\left(c_{0}\right)=c_{0}\right) \wedge\left(g_{1} g_{1}\left(c_{0}\right)=g_{1} g_{1}\left(c_{0}\right)\right)$ is given by $\models_{\mathfrak{A}_{2}}$ and is consequently also true.

However the above contextual model with regard to Remark 3.31 can be seen as too noisy to satisfy condition $v$ for a $\kappa$-Simple contextual model and thus we have no guarantee that we have an Anti-Structure model for the above and certainly a failure to demonstrate simple contextual entanglement. To correct the problem with our example we proceed as follows:

- We will extend our language by adding new function symbols $f_{2}$ and $g_{2}$

- We will extend $\mathfrak{A}_{1}$ and $\mathfrak{A}_{2}$ to the structures $\mathfrak{A}_{1}^{\prime}$ and $\mathfrak{A}_{2}^{\prime}$ where for all $x$, $f_{2}(x)=g_{2}(x)=x$.

- We take $m_{1}^{\prime}=\left\{f_{1}(x), g_{1}(x), f_{1} f_{2} f_{1}(x)\right\}$ and $m_{2}^{\prime}=\left\{f_{1}(x), g_{1}(x), g_{1} g_{2} g_{1}(x)\right\}$.

From the above it is easily seen that we have $\models_{\mathfrak{A}_{1}^{\prime}}\left(\left(f_{1}\left(c_{0}\right)=c_{0}\right) \wedge\left(g_{1}\left(c_{0}\right)=\right.\right.$ $\left.\left.c_{1}\right) \wedge\left(f_{1} f_{2} f_{1}\left(c_{0}\right)=f_{1} f_{2} f_{1}\left(c_{0}\right)\right)\right)$ is true and $\models_{\mathfrak{A}_{2}^{\prime}}\left(\left(f_{1}\left(c_{0}\right)=c_{1}\right) \wedge\left(g_{1}\left(c_{0}\right)=\right.\right.$ $\left.\left.c_{0}\right) \wedge\left(g_{1} g_{2} g_{1}\left(c_{0}\right)=g_{1} g_{2} g_{1}\left(c_{0}\right)\right)\right)$ is true, so that if the above contextual model is also a simple contextual model, then we have an example of simple contextual entanglement. Because $x$ occurs as a sub-term of all of our terms it follows to show that we have a simple contextual model it will suffice for us to show that $\left\{f_{1} f_{2} f_{1}\left(c_{0}\right), f_{1} f_{2} f_{1}\left(c_{1}\right)\right\} \cap \operatorname{Con}\left(m_{2}^{\prime}, \mathfrak{A}_{2}^{\prime}\right)=\varnothing$ and $\left\{g_{1} g_{2} g_{1}\left(c_{0}\right), g_{1} g_{2} g_{1}\left(c_{1}\right)\right\} \cap$ $\operatorname{Con}\left(m_{1}^{\prime}, \mathfrak{A}_{1}^{\prime}\right)=\varnothing$. To see the preceding we need only make note of the fact that $g_{2} \notin m_{1}^{\prime}$ and $f_{2} \notin m_{2}^{\prime}$ and therefore for any constant $c$ the construction of $g_{1} g_{2} g_{1}(c)$ and $f_{1} f_{2} f_{1}(c)$ from $m_{1}^{\prime}$ under $\mathfrak{A}_{1}^{\prime}$ and $m_{2}^{\prime}$ under $\mathfrak{A}_{2}^{\prime}$ respectively, is impossible.

Remark 3.34. In the preceding example we demonstrated that from certain contextual models a simple contextual model can be generated by introducing into existing terms new function symbols. We might think of this as adding buffer terms to lengthen the terms we started with, and remove noise. As the new terms in our example were all assigned the identity function their contribution was only to add a step which made no change in outcome as far as functionality is concerned but did remove terms from having unintended consequences on what we see as true (see Remark 3.31). 
Our next set of definitions and theorem give further utility to the notion of removing noise to generate simple contextual models.

Definition 3.35. A contextual model Contx will be called rational if and and only if it satisfies conditions $i-i v$ for being a simple contextual model and in addition satisfies the following:

If there exists a $c v \in C V a r$ where $t[c v] \in m_{i}[c v] \cap m_{j}[c v]$, for $i \neq j$ and it is not the case that there exists $r \in m_{i}$ where:

$$
a_{1}^{\prime} \cdot r\left[c v^{\prime}\right] \notin m_{j}\left[c v^{\prime \prime}\right] \text { for all }\left\{c v^{\prime}, c v^{\prime \prime}\right\} \subseteq C V a r,
$$

$a_{2}^{\prime}$. and $r$ shares a sub-term with $t$ so that we might have ( $r$ is a sub-term of $t$ ) or ( $t$ is a sub-term of $r$ ),

then there is a constant symbol $b$ where $t^{\mathfrak{A}_{i}}[c v]=b^{\mathfrak{A}_{i}}$ and $t^{\mathfrak{A}_{j}}[c v]=b^{\mathfrak{A}_{j}}$.

Definition 3.36. We give a definition of a measure Slowed for rational contextual models, where Slowed(Contx) is meant to give a measure of how many new function symbols we need to add to the terms of our rational contextual model Contx to produce a meaningful simple contextual model. To these ends we must first define what we will call an interior addition to a term and a consistent alteration of a set of terms by interior additions.

- Given $t$ is a term and $f\left(r_{1}, \ldots, r_{n}\right)$ is a sub-term of $t$, if $t^{\prime}$ is attained from $t$ by replacing the sub-term $f\left(r_{1}, \ldots, r_{n}\right)$ in all of its occurrences in $t$ for at least one $i \leq n$ with $f\left(r_{1}, \ldots, g\left(r_{i}\right) \ldots, r_{n}\right)$ where $g$ did not occur in $t$, then we say $t^{\prime}$ is attained from $t$ with an interior addition of $g$. If $t_{n+1}$ is attained from $t_{n}$ by the interior addition of a $g_{n+1}$ where $t_{1}$ is attained from $t$ with the interior addition of $g_{1}$ and there is no occurrence of $g_{i} g_{j}$ in $t_{n+1}$, then $t_{n+1}$ will be said to be attained from $t$ by the interior addition of $g_{1}, \ldots, g_{n+1}$.

- If $S$ and $S^{\prime}$ are sets of terms where there exists a bijection $L: S \rightarrow S^{\prime}$ so that $L(t)$ is either equal to $t$ or $L(t)$ is attained from $t$ by an interior addition of terms, then we will say that $S^{\prime}$ is a consistent alteration of $S$ by interior additions if and only if given $t_{1}, t_{2} \in S$ where $t_{1}$ is a sub-term of $t_{2}$, we have that for each occurrence of $t_{1}$ in $t_{2}$ there is a corresponding occurrence of $L\left(t_{1}\right)$ in $L\left(t_{2}\right)$ (by corresponding occurrence we mean that given $<$ is the lexicographic order defining the construction of a term from subterms (tree construction), then $L$ preserves $<$ ). We will call $L$ the interior witness map. When we define a map on terms which takes any term $t$ to a term $t^{\prime}$ only if $t^{\prime}$ can be attained from $t$ by interior addition, then we will call this map an interior addition map.

For a rational contextual model Contx having sets of measurables $m_{i}$ for $i \leq \kappa$, we will have Slowed(Contx) $=\delta$ if and only if $\delta$ is the minimum cardinal number needed to attain a simple contextual model from Contx with a consistent alteration of $\bigcup_{i \leq \kappa} m_{i}$ by interior additions of $\delta$ many new function symbols. 
Theorem 3.37. For every rational contextual model Contx there exists a cardinal $\delta$ where Slowed(Contx $)=\delta$.

Proof. Suppose Contx is a rational contextual model having measurables $m_{i}$ for $i<\kappa$. Taking $F$ to be the set of function symbols for the language of Contx, and $F^{\prime}$ the set of terms which consist of function symbols acting on variables. We recursively construct a set of terms as follows:

- $B_{1}=F^{\prime}$

- $B_{n+1}=\left\{f\left(t_{1}^{*}\left(t_{1}\right), \ldots, t_{m}^{*}\left(t_{m}\right)\right) \mid f \in F\right.$ and $\left.\left\{t_{1}, \ldots, t_{m}\right\} \in B_{n} \cup V\right\}$ where for each $t \in B_{n}, t^{*}$ is a new unary function symbol which is added to the language and is distinct for each $t$ and if $t$ is $x$, then $t^{*}(t)$ is also $x$.

- $T^{*}=\bigcup_{n \in \mathbb{N}} B_{n}$

Given $T$ is the set of constant free terms of the language for Contx, we will take $L: T \rightarrow T^{*}$ so that if all new function symbols are removed from $L(t)$, then you have $t$. It is easily checked that $L$ is a bijection. We take $m_{i}^{*}=L\left[m_{i}\right]$ and $\mathfrak{A}_{i}^{*}$ to be the extension of $\mathfrak{A}_{i}$ to the new unary function symbols where $\left(f^{*}\right)^{\mathfrak{A l}_{i}^{*}}(x)=x$. We note the following:

If $t[c v] \in m_{i}^{*}[c v] \cap m_{j}^{*}[c v]$, then $L^{-1}(t)[c v] \in m_{i}[c v] \cap m_{j}[c v]$ and therefore either there exists a constant symbol $b$ where:

i. $t^{\mathfrak{A}_{i}^{*}}[c v]=L^{-1}(t)^{\mathfrak{A}_{i}}[c v]=b^{\mathfrak{A}_{i}}$ and $t^{\mathfrak{A}_{j}^{*}}[c v]=L^{-1}(t)^{\mathfrak{A}_{j}}[c v]=b^{\mathfrak{A}_{j}}$

ii. Or there is an $r \in m_{i}^{*}$ where $L^{-1}(r)$ satisfies conditions $a_{1}^{\prime}$ and $a_{2}^{\prime}$ for $L^{-1}(t)$ of the definition of a rational contextual model.

If in $i i$ above we have $r \in F^{\prime}$, then since for all $c v^{\prime} \in C V a r, r\left[c v^{\prime}\right]=$ $L^{-1}(r)\left[c v^{\prime}\right] \notin m_{j}\left[c v^{\prime}\right]$, we have $r[c v] \notin m_{j}^{*}[c v]$ and therefore $r[c v] \notin$ $\operatorname{Con}\left(m_{j}^{*}, \mathfrak{A}_{j}^{*}\right)$.

Note, $F^{\prime}\left[c v^{\prime}\right] \cap m_{j}^{*}\left[c v^{\prime}\right]=\operatorname{Con}\left(m_{j}^{*}, \mathfrak{A}_{j}^{*}\right) \cap F^{\prime}\left[c v^{\prime}\right]$ by definition.

We note that if $t^{\prime}\left(x_{1}, \ldots, x_{n}\right), t_{1}, \ldots, t_{n} \in T^{*}$, then $t^{\prime}\left(t_{1}, \ldots, t_{n}\right) \notin T^{*}$ because otherwise by construction, each $x_{l}$ in $t^{\prime}\left(x_{1}, \ldots, x_{n}\right)$ is the argument of a function symbol from $F$ occurring in $t^{\prime}$, and then denoting this function symbol $f_{l}$, we have that in $t^{\prime}\left(t_{1}, \ldots, t_{n}\right)$ there is an occurrence of $f_{l}\left(\ldots, t_{l}, \ldots\right)$ which contradicts our construction of $T^{*}$, since being in $T^{*}$ would require $t_{l}^{*}$ to act on $t_{l}$, to have $f_{l}$ composed with $t_{l}$ in any term from $T^{*}$.

We also note that for $t_{1}, t_{2}, t_{3} \in T^{*}$ where $t_{2}$ is a proper sub-term of $t_{1}$, while $t_{2}$ and $t_{3}$ are distinct terms, if $t^{\prime}$ is obtained by substituting $t_{3}$ for $t_{2}$ into $t_{1}$, we have $t^{\prime} \notin T^{*}$, since it contains at least an occurrence of $t_{2}^{*}\left(t_{3}\right)$ and by construction and assumption we have both: if $t_{3}$ occurs as a sub-term of any term of $T^{*}$ it must occur as $t_{3}^{*}\left(t_{3}\right)$; and $t_{2}^{*}$ does not equal $t_{3}^{*}$. 
From the preceding notes we see that if in $i i$ above we have $r \notin F^{\prime}$, then since $r \in T^{*}$ is a composite term and neither a composition of terms in $T^{*}$, nor a non-trivial substitution of a term from $T^{*}$ into another term of $T^{*}$, can give a term in $T^{*}$, we must have $r[c v] \notin \operatorname{Con}\left(m_{j}^{*}, \mathfrak{A}_{j}^{*}\right)$.

In the preceding we see that $\left\{\left\langle m^{*}, \mathfrak{A}_{i}^{*}\right\rangle \mid i<\kappa\right\}$ defines a simple contextual model from the rational contextual model Contx by a consistent alteration by interior additions, and consequently it follows Slowed(Contx) is defined and Slowed(Contx $) \leq\left|T^{*}\right|$.

Proposition 3.38. If Contx is a rational contextual model and Contx* is a simple contextual model constructed from Contx by a consistent alteration by interior additions, then given $L$ is the interior witness map for the aforementioned alteration and given for each formula $\phi$, we take $\phi^{L}$ to be the formula attained from $\phi$ by replacing each $t \in \operatorname{ter}(\phi)-(C \cup V)$ with $L(t)$, then we have for $\phi$ where $\operatorname{ter}(\phi)-(C \cup V)=m_{i}$, $\operatorname{Contx}(\phi, v)=\operatorname{Contx}^{*}\left(\phi^{L}, v\right)$.

Corollary 3.39. For every rational contextual model Contx there exists a term map $L$ and an Anti-Structure $\models_{\mathfrak{A}}^{A}$ where $\left(\models_{\mathfrak{A}}^{A} \pi\left(\phi^{L}[c v]\right)=\operatorname{Contx}(\phi, v)\right.$ when $\langle\phi, v\rangle$ are in the domain of Contx.

For future use, as well as to complete our incorporation of alterations by interior additions we give the following:

Definition 3.40. In a language $\mathfrak{L}$, given Contx $x_{1}$ and Contx $x_{2}$ are simple contextual models given by $\left\{\left\langle m_{i}, \mathfrak{A}_{i}\right\rangle \mid i<\kappa\right\}$ and $\left\{\left\langle w_{i}, \mathfrak{A}_{i}^{\prime}\right\rangle \mid i<\kappa\right\}$ respectively, where for all $m_{i}, w_{j}$ from the aforementioned sets, we have that if $t \in w_{j} \cap m_{i}$ then $t$ includes a non-trivial sub-term (a function symbol with an argument having a function symbol). We then will have $\operatorname{Spaced}\left(\left\{\left\langle w_{i}, \mathfrak{A}_{i}^{\prime}\right\rangle \mid i<\kappa\right\},\left\{\left\langle m_{i}, \mathfrak{A}_{i}\right\rangle \mid i<\kappa\right\}\right)=\delta$ if and only if $\delta$ is the minimum cardinality of the number of new function symbols added to the language $\mathfrak{L}$ to give a consistent alteration of $\left\{\left\langle w_{i}, \mathfrak{A}_{i}^{\prime}\right\rangle \mid i<\kappa\right\}$ by interior additions to yielding $\left\{\left\langle w_{i}^{*},\left(\mathfrak{A}_{i}^{\prime}\right)^{*}\right\rangle \mid i<\kappa\right\}$ where $\left.\left\{\left\langle w_{i}^{*},\left(\mathfrak{A}_{i}^{\prime}\right)^{*}\right\rangle \mid i<\kappa\right\} \cup\left\{\left\langle m_{i}, \mathfrak{A}_{i}\right\rangle \mid i<\kappa\right\}\right)$ gives a simple contextual model. Note that in the preceding each $w_{i}^{*}$ is our alteration of $w_{i}$ by interior additions with $\left(\mathfrak{A}_{i}^{\prime}\right)^{*}$ the extension of $\mathfrak{A}_{i}^{\prime}$ to the language of $w_{i}^{*}$ where all new function symbols are assigned the identity function.

Remark 3.41. We note that in the above $\operatorname{Spaced}\left(\{\langle w, \mathfrak{A}\rangle\},\left\{\left\langle m_{i}, \mathfrak{A}_{i}\right\rangle \mid i<\kappa\right\}\right)$ is easily seen to be well defined because we can make an alteration by interior additions of $w$ much like we did in our proof of Theorem 3.37 and we then would have $\left\{\left\langle w^{*}, \mathfrak{A}^{*}\right\rangle\right\} \cup\left\{\left\langle m_{i}, \mathfrak{A}_{i}\right\rangle \mid i<\kappa\right\}$ gives a simple contextual model, noting that our requirements on $w$ can be less strict than requirements $a_{1}^{\prime}, a_{2}^{\prime}$ for the rational contextual model which was the subject of Theorem 3.37, because we are not requiring that we have a consistent alteration of $\{\langle w, \mathfrak{A}\rangle\} \cup\left\{\left\langle m_{i}, \mathfrak{A}_{i}\right\rangle \mid i<\kappa\right\}$. We then have $\operatorname{Spaced}\left(\left\{\left\langle w_{i}, \mathfrak{A}_{i}^{\prime}\right\rangle \mid i<\kappa\right\},\left\{\left\langle m_{i}, \mathfrak{A}_{i}\right\rangle \mid i<\kappa\right\}\right)$ is similarly well defined. 


\section{Probabilistic Models for Wave Functions and Entanglement}

We now look at contextual models where a formula $\phi$ under a variable assignment $v$ is meant to capture a statement about measured values for instances of execution of a continually repeating experiment. The formula $\phi$ under a variable assignment $v$ is then given a probability of being true which we will take to be

$$
\mu(\phi[v])=\lim _{n \rightarrow \infty} \frac{\text { Number of times } \phi[v] \text { is true in the first } n \text { instances }}{n}
$$

It is important to note that the model we give does not use the measure $\mu$ but allows us to determing the numerator for the argument of the above limit for any given $n$, and then calculation of the argument of the limit from these values will give a sequence that tends to $\mu(\phi[v])$. Note, $\phi$ may be a statement about multiple measurements which occur at different moments in time or at different places in a compound processes. And as such instance of an experiment may refer to an interval of time in which all the required measurements are made. We now formally establish the notion of a probabilistic simple contextual model and give our second model completeness theorem.

Definition 4.1. pContx will be a simple $\kappa$-probabilistic contextual model for a language $\mathfrak{L}$ if and only if each of the following hold:

1. For each $n \in \mathbb{N}$ there exists a $\kappa$ - simple contextual model Contx ${ }_{n}$ for $\mathfrak{L}$ where for all $n, n^{\prime} \in \mathbb{N}$, we have $m_{i}^{n}=m_{i}^{n^{\prime}}$. Where $m_{i}^{j}$ is the $i$ th measurement set for $\operatorname{Contx}_{j}$ for all $j \in \mathbb{N}$. We will also have that $\mathfrak{A}_{i}^{n}$ is the structure where $\operatorname{Contx}_{n}(\phi, v)=\left(\models_{\mathfrak{A}_{i}^{n}} \phi[v]\right)$ when $\operatorname{ter}(\phi)-(C \cup V)=m_{i}^{n}$.

2. When we have a $\phi$ where $\operatorname{ter}(\phi)-(C \cup V)=m_{i}^{n}$ for some $n$, then we will have index $(\phi)=i$ where from the above we then have that if $\operatorname{index}(\phi)=i$, then for all $j \in \mathbb{N}$, ter $(\phi)-(C \cup V)=m_{i}^{j}$

3. Given index $(\phi)=i$ and $v$ is a variable assignment for $\mathfrak{A}_{i}^{n}$, then we will have $\operatorname{pContx}(n, \phi[c v])=\operatorname{Contx}_{n}\left(\phi, v^{\prime}\right)$.

4. We will have $\mu_{\mathrm{pContx}}(\phi[c v])=\lim _{n \rightarrow \infty} \frac{|\{j \mid j \leq n \wedge \operatorname{pContx}(j, \phi[c v])=T\}|}{n}$

Theorem 4.2. Given $\mathrm{pContx}$ is a simple $\kappa$-probabilistic model having language $\mathfrak{L}$, where $\operatorname{pContx}(n, \phi[c v])=\operatorname{Contx}_{n}(\phi, v)$ for $\langle\phi, v\rangle$ in the domain of pContx and $n \in \mathbb{N}$, then there exists:

- A language $\mathfrak{L}^{*}$ which includes $\pi \mathfrak{L}$.

- For each $n \in \mathbb{N}$, an interior addition map $L_{n}$ from the terms of $\mathfrak{L}$ into the terms of $\mathfrak{L}^{*}$.

- And an Anti-Structure $\models_{\mathfrak{A}}^{A}$ for $\mathfrak{L}^{*}$.

Where for all $\langle\phi, v\rangle$ in the domain of $\mathrm{pContx}$ and all $n \in \mathbb{N}$ we have $\operatorname{pContx}(n, \phi[c v])=$ $\left(\models_{\mathfrak{A}}^{A} \pi\left(\phi^{L_{n}}[c v]\right)\right)$ 
Note that $\phi^{L_{n}}$ is the formula given by replacing each $t \in \operatorname{ter}(\phi)-(C \cup V)$ with $L_{n}(t)$.

Proof. Our proof will utilize witnesses to the Spaced operator to give $\models_{\mathfrak{A}}^{A}$ and each $L_{n}$, but before we can implement this construction we must modify each $\left\langle m_{j}^{i}, \mathfrak{A}_{j}^{i}\right\rangle$ to meet our requirements for Spaced to be defined. To these ends we take a new unary function symbol $s$ and we will define:

- For each term $t, t^{s}$ is the term given from $t$ by replacing each occurrence of a variable $x$ in $t$ with $s(x)$, and then we will take $\left(m_{j}^{i}\right)^{\prime}=\left\{t^{s} \mid t \in m_{j}^{i}\right\}$

- For each structure $\mathfrak{A}_{j}^{i}$ we extend the structure to the $\left(\mathfrak{A}_{j}^{i}\right)^{\prime}$ where $s^{\left(\mathfrak{A}_{j}^{i}\right)^{\prime}}(x)=$ $x$.

We note that if $W_{n}=\left\{\left\langle\left(m_{j}^{n}\right)^{\prime},\left(\mathfrak{A}_{j}^{n}\right)^{\prime}\right\rangle \mid j<\kappa\right\}$, then we have satisfied our requirements for $\operatorname{Spaced}\left(W_{n+t}, W_{n}^{*} \cup \ldots \cup W_{1}\right)$ to be defined where for each $i \leq n, W_{i}^{*}$ is given by a consistent alteration by interior additions of $W_{i}$ where $W_{n}^{*} \cup \ldots \cup W_{2}^{*} \cup W_{1}$ then gives a simple contextual model. Taking $L_{n}$ to be the interior addition map for terms $(\mathfrak{L})$ and $W_{n}^{*}$, and taking $\models_{\mathfrak{A}}^{A}$ to be the AntiStructure for the simple contextual model given by $W_{1} \cup \bigcup_{n \in \mathbb{N}} W_{n}^{*}$ we clearly have $\operatorname{pContx}(n, \phi[c v])=\operatorname{Contx}_{n}(\phi, v)=\left(\models_{\mathfrak{A}}^{A} \pi\left(\phi^{L_{n}}[c v]\right)\right)$.

Example 4.3. We wish to give an Anti-Structure model for actions on a compact metric space domain $X$, over instances, where these actions are governed by wave functions and subject to having an interpretation in a superposition, which then collapse to the components of the superposition when observed in an instance. To make things easy we will work with the closed unit sphere $S^{d-1}=X$ in $\mathbb{R}^{d}$. Fixing $d$ we define $\Psi^{*}$ to be the set of all wave functions with domain $S^{d-1}$ and for each $\psi \in \Psi^{*}$ we will take $\delta_{\psi}=\sqrt{\int_{S^{d-1}}|\psi|^{2}}$.

- We will let $L$ be a first order language that contains: the unary function $f_{\psi}$ where $\psi \in \Psi^{*}$, the binary function symbol + , a binary predicate $\mathbf{B}_{\epsilon}$ for all $\epsilon \in \mathbb{R}^{+}$, and for each $\mathbf{v} \in \mathbb{R}^{n}$ a constant symbol $c_{\mathbf{v}}$.

- For $\psi \in \Psi^{*}$ we will take $M_{\langle\psi\rangle}=\left\{f_{\psi}(s), f_{\psi}(s)+s\right\}$. We will then have for each $i \in \mathbb{N}$, that $\mathfrak{A}_{\langle\psi\rangle}^{i}$ will be a structure for our language where $\left|\mathfrak{A}_{\langle\psi\rangle}^{i}\right|=S^{d-1}$ while $f_{\psi}^{\mathfrak{A}_{\langle\psi\rangle}^{i}}\left(c_{\mathbf{0}}\right)=x_{i}^{\psi}$ and $x_{i}^{\psi}$ is the sequence asserted by Proposition 4.4 below.

- For $\langle\psi\rangle$ we will take $\phi_{\epsilon}^{\langle\psi\rangle}(\mathbf{z})$ to be

$$
f_{\psi}\left(c_{\mathbf{0}}\right)+c_{\mathbf{0}}=f_{\psi}\left(c_{\mathbf{0}}\right) \wedge B_{\epsilon}\left(f_{\psi}\left(c_{\mathbf{0}}\right), \mathbf{z}\right)
$$

- For every $\psi, \psi_{1}, \ldots, \psi_{n} \in \Psi^{*}$ where

$$
\psi=\alpha_{1} \psi_{1}+\ldots+\alpha_{n} \psi_{n},
$$


with $\left|\alpha_{i}\right|^{2} \in(0,1)$ and $\sum_{i=1}^{n}\left|\alpha_{i}\right|^{2}=1$, define

$$
\begin{gathered}
M_{\left\langle\psi, \alpha_{1} \psi_{1}, \ldots \alpha_{n} \psi_{n}\right\rangle}= \\
\left\{f_{\psi}(s), f_{\psi_{1}}(s), \ldots, f_{\psi_{n}(s)},\left(\ldots\left(f_{\psi_{1}}(s)+f_{\psi_{2}}(s)\right)+\ldots\right)+f_{\psi_{n}}(s)\right\}
\end{gathered}
$$

- Define the following formulas:

$$
\begin{aligned}
\phi_{i} \text { is the formula } & \left(\left(f_{\psi}\left(c_{\mathbf{0}}\right)=f_{\psi_{i}}\left(c_{\mathbf{0}}\right)\right) \wedge \bigwedge_{j \neq i}\left(f_{\psi_{j}}\left(c_{\mathbf{0}}\right)=c_{\mathbf{0}}\right)\right) \wedge \\
& \left.\left(f_{\psi}\left(c_{\mathbf{0}}\right)=\left(\ldots\left(f_{\psi_{1}}\left(c_{\mathbf{0}}\right)+f_{\psi_{2}}\left(c_{\mathbf{0}}\right)\right)+\ldots\right)+f_{\psi_{n}}\left(c_{\mathbf{0}}\right)\right)\right) \\
\phi_{i, \epsilon}(z) \text { is the formula } & \phi_{i} \wedge \mathbf{B}_{\epsilon}\left(f_{\psi}\left(c_{\mathbf{0}}\right), z\right) \\
\phi_{\epsilon}(z) \text { is the formula } & \left(f_{\psi}\left(c_{\mathbf{0}}\right)=\left(\ldots\left(f_{\psi_{1}}\left(c_{\mathbf{0}}\right)+f_{\psi_{2}}\left(c_{\mathbf{0}}\right)\right)+\ldots\right)+f_{\psi_{n}}\left(c_{\mathbf{0}}\right)\right) \wedge \\
& \bigwedge_{j \leq n}\left(f_{\psi_{j}}\left(c_{\mathbf{0}}\right)=f_{\psi_{j}}\left(c_{\mathbf{0}}\right)\right) \wedge \mathbf{B}_{\epsilon}\left(f_{\psi}\left(c_{\mathbf{0}}\right), z\right) .
\end{aligned}
$$

- Given the following two propositions:

Proposition 4.4. For any $\psi \in \Psi^{*}$, there exists $x^{\psi}: \mathbb{N} \rightarrow S^{d-1}$ such that for any $\epsilon \in \mathbb{R}^{+}$and any $\mathbf{z} \in S^{d-1}$ we have

$$
\int_{\|\mathbf{v}-\mathbf{z}\|<\epsilon}\left|\frac{\psi(\mathbf{v})}{\delta_{\psi}}\right|^{2} d \mathbf{v}=\lim _{m \rightarrow \infty} \frac{\left|\left\{j:\left\|x_{j}^{\psi}-\mathbf{z}\right\|<\epsilon\right\} \cap[m]\right|}{m} .
$$

Proposition 4.5. For complex $\alpha_{1}, \ldots, \alpha_{n}$ where $\sum_{j=1}^{n}\left|\alpha_{j}\right|^{2}=1$ there exists disjoint subsets $T_{1}, \ldots, T_{n}$ of $\mathbb{N}$ where $\bigcup_{j=1}^{n} T_{j}=\mathbb{N}$, and for any $i \in\{1, \ldots, n\}$,

$$
\lim _{m \rightarrow \infty} \frac{\left|T_{i} \cap[m]\right|}{m}=\left|\alpha_{i}\right|^{2}
$$

Now for $M_{\left\langle\psi, \alpha_{1} \psi_{1}, \ldots \alpha_{n} \psi_{n}\right\rangle}$, we take $T_{1}, \ldots, T_{n}$ from Proposition $4.5, x^{\psi_{1}}, \ldots, x^{\psi_{n}}$ from Proposition 4.4, and for each $i \leq n$ take an order preserving bijection $g_{i}: T_{i} \rightarrow \mathbb{N}$ and define $\mathfrak{A}_{\left\langle\psi, \alpha_{1} \psi_{1}, \ldots \alpha_{n} \psi_{n}\right\rangle}^{l}$ for $l \in T_{i}$ so that

1. $\left|\mathfrak{A}_{\left\langle\psi, \alpha_{1} \psi_{1}, \ldots \alpha_{n} \psi_{n}\right\rangle}^{l}\right|=S^{d-1} \cup\{\mathbf{0}\}$

2. $f_{\psi}^{\mathfrak{A}_{\left\langle\psi, \alpha_{1} \psi_{1}, \ldots \alpha_{n} \psi_{n}\right\rangle}^{l}}\left(c_{\mathbf{0}}\right)=f_{\psi_{i}}^{\mathfrak{A}_{\left\langle\psi, \alpha_{1} \psi_{1}, \ldots \alpha_{n} \psi_{n}\right\rangle}^{l}}\left(c_{\mathbf{0}}\right)=x_{g_{i}(l)}^{\psi_{i}}$,

3. for $j \neq i, f_{\psi_{j}}^{\mathfrak{A}_{\left\langle\psi, \alpha_{1} \psi_{1}, \ldots \alpha_{n} \psi_{n}\right\rangle}^{l}}\left(c_{\mathbf{0}}\right)=\mathbf{0}$.

The preceding gives us a pContx model, and applying Theorem 4.2 gives an Anti-Structure $\models_{\mathfrak{A}}^{A}$, where:

* For $\left\langle\psi, \alpha_{1} \psi_{1}, \ldots \alpha_{n} \psi_{n}\right\rangle$ we have,

1. $\left|\alpha_{i}\right|^{2}=\mu_{\mathrm{pContx}}\left(\phi_{i}[c v]\right)=\lim _{m \rightarrow \infty} \frac{\left|\left\{j: \models_{\mathfrak{A}}^{A} \pi\left(\phi_{i}^{L_{j}}[c v]\right)=T\right\} \cap[m]\right|}{m}$, 


$$
\begin{aligned}
& \text { 2. }\left|\alpha_{i}\right|^{2} \int_{\|\mathbf{v}-v(z)\|<\epsilon}\left|\frac{\psi_{i}(\mathbf{v})}{\delta_{\psi_{i}}}\right|^{2} d \mathbf{v}=\mu_{\mathrm{pContx}}\left(\phi_{i, \epsilon}[c v]\right) \\
& =\lim _{m \rightarrow \infty} \frac{\left|\left\{j: \mid \models_{\mathfrak{A}}^{A} \pi\left(\phi_{i, \epsilon}^{L_{j}}[c v]\right)=T\right\} \cap[m]\right|}{m} \\
& \text { 3. } \sum_{j=1}^{n} \int_{\|\mathbf{v}-v(z)\|<\epsilon}\left|\alpha_{j}\right|^{2}\left|\frac{\psi_{j}(\mathbf{v})}{\delta_{\psi_{j}}}\right|^{2} d \mathbf{v}=\mu_{\mathrm{pContx}}\left(\phi_{\epsilon}[c v]\right) \\
& =\lim _{m \rightarrow \infty} \frac{\left|\left\{j: \models_{\mathfrak{A}}^{A} \pi\left(\phi_{\epsilon}^{L_{j}}[c v]\right)=T\right\} \cap[m]\right|}{m} . \\
& \text { * }\left.\operatorname{For}_{\text {4. }\langle\psi\rangle} \int_{\|\mathbf{v}-v(z)\|<\epsilon} \operatorname{have}_{\delta_{\psi}}\right|^{2} d \mathbf{v}=\mu_{\mathrm{pContx}}\left(\phi_{\epsilon}^{\langle\psi\rangle}[c v]\right) \\
& =\lim _{m \rightarrow \infty} \frac{\left|\left\{j: \models_{\mathfrak{A}}^{A} \pi\left(\left(\phi_{\epsilon}^{\langle\psi\rangle}\right)^{L_{j}}[c v]\right)=T\right\} \cap[m]\right|}{m} .
\end{aligned}
$$

In $\models_{\mathfrak{A}}^{A}$, we have $f_{\psi}\left(c_{\mathbf{0}}\right), f_{\psi_{1}}\left(c_{\mathbf{0}}\right), \ldots, f_{\psi_{n}}\left(c_{\mathbf{0}}\right)$ are tangible actions taking $\mathbf{0}$ to a place on $S^{d-1}$, where then $\left\langle\psi, \alpha_{1} \psi_{1}, \ldots \alpha_{n} \psi_{n}\right\rangle$ representing the superposition $\psi=\sum_{j=1}^{n}\left(\alpha_{j}\right) \psi_{j}$, and gives that $\phi_{i}, \phi_{i, \epsilon}, \phi_{\epsilon}$ are formulas talking about $f_{\psi}\left(c_{\mathbf{0}}\right), f_{\psi_{1}}\left(c_{\mathbf{0}}\right), \ldots, f_{\psi_{n}}\left(c_{\mathbf{0}}\right)$ and representing the superposition, where in $\models_{\mathfrak{A}}^{A}$ we have:

- $\phi_{\epsilon}$ yields that $f_{\psi}$ has probability distribution consistent with it behaving from instance to instance like it is under exactly one of the probability distributions given by an $\alpha_{i} \psi_{i}$.

- $\phi_{i}$ yields that with frequency $\left|\alpha_{i}\right|^{2}, f_{\psi}$ is $f_{\psi_{i}}$ with $f_{\psi_{j}}=\mathbf{0}$ for $j \neq i$

- $\phi_{i, \epsilon}$ yields that the probability distribution of $f_{\psi}$ when it behaves like $f_{\psi_{i}}$ is consistent with the probability distribution given by $\psi_{i}$.

Under $\langle\psi\rangle, \phi_{\epsilon}^{\langle\psi\rangle}$ talks only about $f_{\psi}\left(c_{\mathbf{0}}\right)$ and is not measuring it in the components of a super position, and in this case we have that the probability distribution of $f_{\psi}$ is consistent with $\psi$ and consequently has a distribution from instance to instance which is consistent with any interference occurring under $\psi$. And thus we have that in $\models_{\mathfrak{A}}^{A}, \phi_{i}, \phi_{i, \epsilon}, \phi_{\epsilon}$ are very much asserting that the action $f_{\psi}\left(c_{0}\right)$ when not measured in the components of a superposition is governed by $\psi$ but when taken to be in a superposition of $f_{\psi_{1}}, \ldots, f_{\psi_{n}}$ and measured as such, is from instance to instance acting like exactly one of the $f_{\psi_{i}}$ 's in a manner consistent with the collapse of the given superposition under measurement.

Remark 4.6. The significance of Example 4.3 is in the fact that the AntiStructure $\models_{\mathfrak{A}}^{A}$ which is discerning truth solely on the basis of the defined states of its terms, represents all the contexts in which we have an action $f_{\psi}\left(c_{\mathbf{0}}\right)$ in a superposition of actions $f_{\psi_{1}}\left(c_{\mathbf{0}}\right), \ldots, f_{\psi_{n}}\left(c_{\mathbf{0}}\right)$, where under measurement the superposition collapses to exactly one of the actions and when under less strict measurement exhibits interference. With Example 4.3 we have shown that even the probabilistic nature of context change in quantum mechanics can be modeled 
solely by where the terms of discourse are defined under the assumption that there is absolutely no information where they are not defined. To show how context change is captured by Example 4.3 we point out that if we had:

- We take normalized wave functions $\psi_{A}$ and $\psi_{B}$ representing the transition of a photon from a source passing from intermediate slits $A$ and $B$ (in a barrier) to a back wall respectively.

- We take $\psi=\frac{1}{\sqrt{2}} \psi_{A}+\frac{1}{\sqrt{2}} \psi_{B}$.

- We will take $\phi_{\epsilon}$ and $\phi_{\epsilon}^{\langle\psi\rangle}$ as given above for $\left\langle\psi, \frac{1}{\sqrt{2}} \psi_{A}, \frac{1}{\sqrt{2}} \psi_{B}\right\rangle$ and $\langle\psi\rangle$

* It follows:

- When $\mathbf{z}$ ranges over the back-wall, $\phi_{\epsilon}^{\langle\psi\rangle}(\mathbf{z})$ gives the interference pattern on the back-wall without observation at $A$ or $B$.

- When $\mathbf{z}$ ranges over the back-wall, $\phi_{\epsilon}(\mathbf{z})$ gives the sum of the Gaussian distributions defined by $\left|\frac{1}{\sqrt{2}} \psi_{A}\right|^{2}$ and $\left|\frac{1}{\sqrt{2}} \psi_{B}\right|^{2}$.

Example 4.7. We can capture a probabilistic example of entanglement by making a slight alteration to the example outlined in Remark 4.6. The alteration will model the consequences of measuring or not measuring a photon which is entangled with the photon that transitions from the source through the slits $A$ and $B$. The entanglement will occur at the slit of transition. We take $f_{\psi}$, $f_{\psi_{A}}, f_{\psi_{B}}$ and pContx $x_{1}$ to be the terms and the probabilistic simple contextual model given in Remark 4.6. We will take $g_{\psi}, g_{\psi_{A}}, g_{\psi_{B}}$ and pContx ${ }_{2}$ to copy $f_{\psi}$, $f_{\psi_{A}}, f_{\psi_{B}}$ and $\mathrm{pContx}_{1}$ on a unique point set. For clarity we will superscript a 1 and 2 respectivly for the measurement sets for $\mathrm{pContx}_{1}$ and $\mathrm{pContx}_{2}$. We will take:

- $M_{\left\langle\psi, \psi_{A}, \psi_{B}\right\rangle}^{1 *}=M_{\left\langle\psi, \psi_{A}, \psi_{B}\right\rangle}^{1 *} \cup\{g(s)\}$ and $M_{\left\langle\psi, \psi_{A}, \psi_{B}\right\rangle}^{2 *}=M_{\left\langle\psi, \psi_{A}, \psi_{B}\right\rangle}^{2} \cup\{f(s)\}$

- We will take the structures for $M_{\left\langle\psi, \psi_{A}, \psi_{B}\right\rangle}^{1 *}$ and $M_{\left\langle\psi, \psi_{A}, \psi_{B}\right\rangle}^{2 *}$ the union of the structures given for $M_{\left\langle\psi, \psi_{A}, \psi_{B}\right\rangle}^{1}$ and $M_{\left\langle\psi, \psi_{A}, \psi_{B}\right\rangle}^{2}$ respectively.

- We will define $M^{*}=\{f(s), g(s)\}$.

- The structure for $M^{*}=\{f(s), g(s)\}$ will be the union of the structures for $M_{\langle\psi\rangle}^{1}$ and $M_{\langle\psi\rangle}^{1}$.

From our prior work the above gives a pContx model which in tern has an AntiStructure model $\models_{\mathfrak{A}}^{A}$. Taking ${ }^{1} \phi_{\epsilon}^{\langle\psi\rangle},{ }^{2} \phi_{\epsilon}^{\langle\psi\rangle}$ to be $\phi_{\epsilon}^{\langle\psi\rangle}$ in $\mathrm{pContx}_{1}$ and $\mathrm{pContx}_{2}$ respectively. We will have:

- $\phi_{\epsilon, \delta}^{f, g}(x, y)$ be $\left({ }^{1} \phi_{\epsilon}^{\langle\psi\rangle}(x)\right) \wedge\left({ }^{2} \phi_{\delta}^{\langle\psi\rangle}(y)\right)$

- $\phi_{\epsilon}^{f}(x)$ be

$$
\left({ }^{1} \phi_{\epsilon}^{\langle\psi\rangle}(x)\right) \wedge\left(g_{\psi}\left(c_{\mathbf{0}}\right)=g_{\psi_{A}}\left(c_{\mathbf{0}}\right)+g_{\psi_{B}}\left(c_{\mathbf{0}}\right)\right) \wedge\left(g_{\psi_{A}}\left(c_{\mathbf{0}}\right)=g_{\psi_{A}}\left(c_{0}\right)\right) \wedge\left(g_{\psi_{B}}\left(c_{\mathbf{0}}\right)=g_{\psi_{B}}\left(c_{\mathbf{0}}\right)\right)
$$


- $\phi_{\epsilon}^{g}(x)$ be

$$
\left({ }^{2} \phi_{\epsilon}^{\langle\psi\rangle}(x)\right) \wedge\left(f_{\psi}\left(c_{\mathbf{0}}\right)=f_{\psi_{A}}\left(c_{\mathbf{0}}\right)+f_{\psi_{B}}\left(c_{\mathbf{0}}\right)\right) \wedge\left(f_{\psi_{A}}\left(c_{\mathbf{0}}\right)=f_{\psi_{A}}\left(c_{0}\right)\right) \wedge\left(f_{\psi_{B}}\left(c_{\mathbf{0}}\right)=f_{\psi_{B}}\left(c_{\mathbf{0}}\right)\right)
$$

The pContx and its corresponding Anti-Structure gives:

- Letting $x$ and $y$ range over the back walls, $\phi_{\epsilon, \delta}^{f, g}(x, y)$ will give a probability distribution corresponding to having interference on each wall.

- Letting $x$ range over the back wall for $f_{\psi}$ and $y$ range over the back wall for $g_{\psi}, \phi_{\epsilon}^{f}(x)$ and $\phi_{\epsilon}^{g}(y)$ give respectively the probability distributions corresponding to measuring $f_{\psi}$ and $g_{\psi}$ at the slits $A$ and $B$, where $\phi_{\epsilon}^{f}(x)$ and $\phi_{\epsilon}^{g}(y)$ make statements about measuring $g_{\psi}$ and $f_{\psi}$ at the slits respectively and distinctly. In summary the formulas assert that a measurement of $g_{\psi}$ at the slits destroys interference of $f_{\psi}$ and a measurement of $f_{\psi}$ at the slits destroys the interference for $g_{\psi}$.

\section{Pathological Examples, Comments, and Further Questions}

We give two examples that highlight properties of Anti-Structures which are pathological to standard structures but seem to be consistent with perceptions of the world around us. These properties are:

1. We make statements about the universe and check the truth of these statements, where we being a part of the universe then yields that the universe can make statements about itself and check the truth of those statements.

2. Finite things like a table top or a drawn line segment, respectively appear to be the infinite point sets found as a rectangle in the Euclidean plane and an interval in the real number line, until we make more rigorous or intense observation of them.

The reader may disagree with the above being standard perceptions, and to any such dissent we give no counter argument, however even under such a disagreement, we believe that we can come to the agreement that a mathematical structure which can determine its own truths, is pathological to Gödel's incompleteness theorem, and that a finite structure for a theory where every standard model of the theory has an infinite point set, is clearly pathological to all the standard structures.

\subsection{Pathological Examples}

Example 5.1. We give an Anti-Structure to address 1 above. We take $\models_{N}$ to be our standard natural numbers as a model of the axioms $A_{E}$, ( see [1]) where for each natural number $n \in|N|$ we have a constant $c_{n}$ where $c_{n}^{N}=n=\left(S^{n}(0)\right)^{N}$. We add to our language a unary function symbol $F$ and a binary function symbol $G$, and give a recursive construction of a system $Y$ for our Anti-Structure as follows: 
In the meta-theory, we take a function $\mathfrak{F}$ mapping the quantifier free formulas of our extended language, bijectivly onto the constant symbols of our language.

We extend $\models_{N}$ to a structure $\models_{N^{\prime}}$ to include $G$, where then

$$
(G(\mathfrak{F}(\phi(x)), c))^{N^{\prime}}=\mathfrak{F}(\phi(c))^{N^{\prime}}
$$

We will take $\Sigma_{0}$ to be the set of variable free formulas of the language of $\models_{N^{\prime}}$ and we will take $Y_{0}=\left\{\phi \in \Sigma_{0} \mid\left(\models_{N^{\prime}} \phi\right)=T\right\}$.

We take $\Delta_{n}=\left\{F(\mathfrak{F}(\phi))=i \mid\left(\phi \in \Sigma_{n}\right) \wedge(i \in\{0,1\}) \wedge\left(i=1 \leftrightarrow \phi \in Y_{n}\right)\right\}$

$Y_{n+1}$ will be the variable free formulas which are the consequences of $\Delta_{n} \cup Y_{n}$ and $\Sigma_{n+1}$ will be the set of all variable free formulas $\phi$ where for each $t \in \operatorname{ter}(\phi)$ there exist a $\psi \in Y_{n+1}$ where $t \in \operatorname{ter}(\psi)$.

We take $Y=\bigcup_{n \in \mathbb{N}} Y_{n}$

$Y$ is easily seen to be a system from its construction and therefore there exists an Anti-Structure $\models_{\mathfrak{A}}^{A}$ for $Y$.

For all variable free formulas $\phi$ we now have $\left(\models_{\mathfrak{A}}^{A} \phi\right)=\left(\models_{\mathfrak{A}}^{A} F(\mathfrak{F}(\phi))=1\right)$ where for all quantifier free formulas $\phi(x)$ we also have $(G(\mathfrak{F}(\phi(x)), c))^{\mathfrak{A}}=\mathfrak{F}(\phi(c))^{\mathfrak{A}} \in A$. We also have $\models_{\mathfrak{A}}^{A} A_{E}$.

We note that the above example does not account for all sentences of our language, however its pathological behavior with regard to Gödel's incompleteness theorem can easily be seen as follows: if $\models_{\mathfrak{B}}$ is a structure and $\mathfrak{F}$ maps all quantifier free formulas into the constant symbols for the language of $\mathfrak{B}$ where there exists: a special constant symbol 1, unary function symbol $F$ and a binary function symbol $G$ in our language, so that:

i. $(G(\mathfrak{F}(\phi(x)), c))^{\mathfrak{B}}=\mathfrak{F}(\phi(c))^{\mathfrak{B}}$

ii. For all variable free formulas $\phi,\left(\models_{B} \phi\right)=\left(\models_{B} F(\mathfrak{F}(\phi))=1\right)$

then taking $\phi(x)$ to be the formula $F(G(x, x)) \neq 1$ and $c=\mathfrak{F}(\phi(x))$, we have

$$
\left(\models_{\mathfrak{B}} \phi(c)\right)=\left(\models_{B} F(\mathfrak{F}(\phi(c)))=1\right)
$$

and

$$
\left(\models_{\mathfrak{B}} \phi(c)\right)=\left(\models_{\mathfrak{B}} F(G(c, c)) \neq 1\right)=\left(\models_{\mathfrak{B}} F(\mathfrak{F}(\phi(c))) \neq 1\right)
$$

and therefore

$$
\left(\models_{\mathfrak{B}} F(\mathfrak{F}(\phi(c)))=1\right)=\left(\models_{\mathfrak{B}} F(\mathfrak{F}(\phi(c))) \neq 1\right)
$$

which is a contradiction. 
Example 5.2. We will take our language to be $\mathfrak{L}$ where the non-logical symbols will be:

* Unary function symbol $S$

* Binary function symbols,$+ \cdot$ and $E$

* Binary predicat symbol <

* For each $n$ in the natural numbers a constant symbol $c_{n}$

we will take $\mathfrak{N}$ to be the standard model of the natural numbers as given in Enderton [1], where we have extended $\mathfrak{N}$ so that $c_{n}^{\mathfrak{N}}=n$. We take $A_{E}$ from [1], to be the following axioms:

$$
\begin{aligned}
& S_{1} . \forall x\left[S(x) \neq c_{0}\right] \\
& S_{2} . \forall x \forall y[(S(x)=S(y)) \rightarrow(x=y)] \\
& L_{1} . \forall x \forall y[(x<S(y)) \leftrightarrow((x<y) \vee(x=y))] \\
& L_{2} . \forall x\left[x \nless c_{0}\right] \\
& L_{3} . \forall x \forall y[(x<y) \vee(x=y) \vee(y<x)] \\
& A_{1} . \forall x\left[x+c_{0}=x\right] \\
& A_{2} . \forall x \forall y[x+S(y)=S(x+y)] \\
& M_{1} . \forall x\left[x \cdot c_{0}=c_{0}\right] \\
& M_{2} . \forall x \forall y[x \cdot S(y)=(x \cdot y)+x] \\
& E_{1} . \forall x\left[x E 0=c_{1}\right] \\
& E_{2} . \forall x \forall y[x E S(y)=(x E y) \cdot x]
\end{aligned}
$$

We now have for all $n \in \mathbb{N}=|\mathfrak{N}|$ it can be seen that $\models_{\mathfrak{N}}^{\{1, \ldots, n\}} A_{E}$ by noting the following:

- Taking all variables to have values taken in $\{1, \ldots, n\}$ the instances of the axioms $L_{2}, L_{3}, A_{1}$ for these values are all seen to be true under $\models_{\mathfrak{N}}^{\{1, \ldots, n\}}$. Note that an instance of one of our axioms is an unquantified formula given by taking the entire formula occurring after the quantification from one of our axioms. Also note that if all of the instances of $L_{2}, L_{3}, A_{1}$ are true under variable assignments into $\{1, \ldots, n\}$, then $L_{2}, L_{3}, A_{1}$ are true under $\models_{\mathfrak{N}}^{\{1, \ldots, n\}}$

- Taking all variable assignments to have values taken in $\{1, \ldots, n\}$ we have that the instances of $S_{1}, S_{2}, L_{1}$ only have a truth value in $\models_{\mathfrak{N}}^{\{1, \ldots, n\}}$ when $S(n)$ does not occur in the instance under the variable assignment, and when $S(n)$ does not occur the instance under the variable assignment is true under $\models_{\mathfrak{N}}^{\{1, \ldots, n\}}$. From the preceding we have that $S_{1}, S_{2}, L_{1}$ are all true under $\models_{\mathfrak{N}}^{\{1, \ldots, n\}}$. 
- Taking all variable assignments to have values taken in $\{1, \ldots, n\}$ we have that the instances of $A_{2}, M_{2}, E_{2}$ have truth value when respectively $x+S(y), x \cdot S(y)$ and $x E S(y)$ are in $\{1, \ldots, n\}$ under the given variable assignment, and when they are defined they are true under $\models_{\mathfrak{N}}^{\{1, \ldots, n\}}$. From the preceding we have $A_{2}, M_{2}, E_{2}$ are all true under $\models_{\mathfrak{N}}^{\{1, \ldots, n\}}$.

From the above we have that $\models_{\mathfrak{N}}^{\{1, \ldots, n\}}$ is a finite Anti-Structure model for $A_{E}$ where every standard model of $A_{E}$ must be infinite. It is important to note that there is no formula $\phi$ where $\models_{\mathfrak{R}}^{\{1, \ldots, n\}} \phi$ being true or false would tell us that we are in a finite point set, and in this regard if $=_{\mathfrak{N}}^{\{1, \ldots, n\}}$ is a tool telling Peter what he sees, then given any axiom that is not $M_{1}$ or $E_{1}$, Peter can not determine the truth of any term substitution into an instance of the axiom beyond the term taking some value before $n$, and as such $n$ represents an upper bound on Peters ability to observe things. We note that axioms $M_{1}$ and $E_{1}$ are different because even though $S^{j}\left(c_{n}\right)^{\mathfrak{N}} \notin\{1, \ldots, n\}$ for any $j>0$ we have $S^{j}\left(c_{n}\right) \cdot c_{0}=c_{0}$ and $S^{j}\left(c_{n}\right) E c_{0}=c_{1}$ are always true under $\models_{\mathfrak{N}}^{\{1, \ldots, n\}}$. We suppose one might debate to what degree a real person Peter would find they have such a bounding number $n$ on their ability to witness instances of the axioms $A_{E}$ in the real world, and consequently we deliberately avoid making any deeper connection between our example and the real world save to note that in both finite things can seem infinite until they are more rigorously inspected.

Before we proceed with comments on the results and examples in this paper, we first give definition to some terms which will allow us to generalize the results and highlight the significance of the last two examples.

Definition 5.3. Given a first order language $L,\langle\Sigma, X\rangle$ will be a sound codified semantic truth assignment for $L$ if and only if

i. $X$ is a point set.

ii. Given Form is the set of formulas for $L, V$ is the set of variables of $L$ and $\mathbb{V}=\{v \mid v: V \rightarrow X\}$, we will have $\Sigma: S \rightarrow\{T, F\}$ with $S \subseteq F$ orm $\times \mathbb{V}$

iii. For each $a \in X$ there exists a constant $c_{a}$ of $L$ where for all variable assignments $v$ we have $\left\langle c_{a}=x, v\right\rangle \in S$. We will also have $\sigma\left(c_{a}=x, v\right)=T$ if and only if $v(x)=a$

iv. If $\langle\psi, v\rangle \in S$ where $\psi$ has no free variables, we will have $\{\psi\} \times \mathbb{V} \subseteq S$ where for all $v_{1}, v_{2} \in \mathbb{V}, \Sigma\left(\psi, v_{1}\right)=\Sigma\left(\psi, v_{2}\right)$ and in this case we will write $\Sigma(\psi)$ for $\Sigma(\psi, v)$.

v. Given $S^{*}=\{\psi \mid \psi$ is variable free and there exists $\langle\psi, v\rangle \in S\}$, and $Y=$ $\left\{\psi \in S^{*} \mid \Sigma(\psi)=T\right\}=\left\{\psi \in S^{*} \mid \Sigma(\neg \psi)=F\right\}$, we will have $Y=\operatorname{cn}(Y) \cap S^{*}$ (when $Y \subsetneq c n(Y) \cap S^{*}$ we say $\Sigma$ is not sound), where $c n(Y)$ is the set of logical consequences of $Y$.

We will let $S^{\prime}=\{\psi \mid \psi$ has no free variables and there exists $\langle\psi, v\rangle \in S\}$. 
For sound and not sound semantic contextual truth assignments $\Sigma$ we will call:

- $X$ the $\Sigma$ universe

- $Y$ the $\Sigma$ system

- $\mathbb{T}_{\Sigma}=\left\{\psi \in S^{\prime} \mid \Sigma(\psi)=T\right\}$

- $\Sigma$ non-trivial if $Y$ removing the logically valid formulas gives a non-empty set

- $\Sigma$ total if $S=$ form $\times \mathbb{V}$

- $\Sigma$ will be atomic total if $\{\psi \mid \psi$ is an atomic formula of $L\} \times \mathbb{V} \subseteq S$

- A sound $\Sigma$ is non-contextual when $c n\left(\mathbb{T}_{\Sigma}\right)$ is a proper subset of the set of formulas of $L$.

We note that any codified Anti-Structure $\models_{\mathfrak{A}}^{A}$ is a codified semantic truth assignment where $A$ is the universe of $\models_{\mathfrak{Q}}^{A}$, the system as given in Definition 3.14 is the system for $\models_{\mathfrak{A}}^{A}$ as given in the preceding Definition 5.3.

Definition 5.4. Given $\langle\Sigma, X\rangle$ is a codified semantic truth assignment for $L$. A codified semantic truth assignment $\left\langle\Sigma^{*}, X\right\rangle$ for $L$ will be expressible in $\langle\Sigma, X\rangle$ if and only if each of the following hold:

- $\left(\Sigma^{*}(\phi, v)=T\right) \Rightarrow(\Sigma(\phi, v)=T)$

- Given $C$ is the set of constant symbols for $L$ and taking $Y^{*}$ and $Y$ to be the systems for $\left\langle\Sigma^{*}, X\right\rangle$ and $\langle\Sigma, X\rangle$ respectively, we will have that there exists a formula $\sigma(x)$ of $L$, a binary function symbol $h$ of $L$ and a function $f$ from the formulas of $L$ into $C$ where:

i. $Y^{*}=\{\psi \in Y \mid \Sigma(\sigma(f(\psi)))=T\}$

ii. For all quantifier free formulas $\phi\left(x_{1}, \ldots, x_{n}\right)$ and all constant symbols $c$,

$$
\left(\Sigma\left(h\left(f\left(\phi\left(x_{1}, \ldots, x_{n}\right)\right), c\right)=f\left(\phi\left(x_{1}, \ldots, c\right)\right)\right)\right)=T
$$

. Note here we mean the notation $\phi\left(x_{1}, \ldots, x_{n}\right)$ to inform us that $x_{1}, \ldots, x_{n}$ is the list of all the unquantified variables of $\phi$ where reading $\phi$ left to write gives the first occurrence of $x_{i}$ is before $x_{j}$ if $i<j$. Furthermore if $\eta$ is $\phi\left(x_{1}, \ldots, x_{n}\right)$, then here we would be free to write $\eta\left(x_{1}, \ldots, x_{n-1}\right)$.

Additionally we will say that a formula $\sigma(x)$ and binary function $h$ witnesses the expressibility of $\left\langle\Sigma^{*}, X\right\rangle$ in $\langle\Sigma, X\rangle$ when $\sigma(x), h,\left\langle\Sigma^{*}, X\right\rangle$ and $\langle\Sigma, X\rangle$ satisfy the above for $\left\langle\Sigma^{*}, X\right\rangle$ being expressible in $\langle\Sigma, X\rangle$.

Definition 5.5. We will call a codified semantic truth assignment $\langle\Sigma, X\rangle$ for a language $L$, expressive if and only if there exists formulas $\sigma_{1}(x), \sigma_{2}(x, y, z)$ in the language $L$, a unary function symbol $g$, a binary function $h$ (which may or may not occur in $L$ ), and a codified semantic truth assignment $\left\langle\Sigma^{*}, X\right\rangle$ for $L \cup\{g, h\}$ where each of the following hold: 
- For all formulas $\phi$ of $L$ and all variable assignments $v$,

$$
\left(\Sigma^{*}(\phi, v)=T\right) \Leftrightarrow(\Sigma(\phi, v)=T)
$$

- There is a constant symbol $c$ in $L$ where for all variable assignments $v$ we have

$$
\left(\Sigma^{*}(g(x)=c, v)\right)=\left(\Sigma\left(\sigma_{1}(x), v\right)\right)
$$

- It is the case that for all variable assignments $v$,

$$
\left(\Sigma^{*}(h(x, y)=z, v)=T\right) \Leftrightarrow\left(\Sigma\left(\sigma_{2}(x, y, z), v\right)=T\right)
$$

- $g(x)=c$ and $h$ witnesses the expressibility of $\left\langle\Sigma^{*}, X\right\rangle$ in itself.

We will call $\Sigma^{*}$ the expressive resolution for $\Sigma$.

Definition 5.6. Given $\left\langle\Sigma_{1}, X\right\rangle$ is a codified semantic truth assignment for a language $L$, we will say that a codified semantic truth assignment $\left\langle\Sigma_{2}, X\right\rangle$ is in existential crisis under $\left\langle\Sigma_{1}, X\right\rangle$ when the $\Sigma_{2}$ system $Y$ is represented in $\left\langle\Sigma_{1}, X\right\rangle$ but $\left\langle\Sigma_{2}, X\right\rangle$ is not expressed in $\left\langle\Sigma_{1}, X\right\rangle$.

\subsection{Comments}

1. Our Example 5.1 gives an Anti-Structure $\models_{\mathfrak{A}}^{A}$ where $\models_{\mathfrak{A}}^{A} c n\left(A_{E}\right)$ and where from the above we also have $\models_{\mathfrak{A}}^{A}$ is expressive. From Example 5.1 we also have the following simple theorems:

Theorem 5.7. For all codified semantic truth assignments $\langle\Sigma, X\rangle$ it is the case that $\langle\Sigma, X\rangle$ does not have an atomic complete expressive resolution.

Corollary 5.8. For all total non-contextual codified semantic truth assignments $\langle\Sigma, X\rangle$ it is the case that $\langle\Sigma, X\rangle$ is not expressive.

I. Assuming $\models_{\mathfrak{N}}$ is the codified structure for the natural numbers, and $\sharp$ represents the Gödel numbering for the language of $\models_{\mathfrak{N}}$, we can attain much of Gödels incompleteness from Corollary 5.8 by noting that the corollary readily produces a contradiction with the assumption that there exists a formula $\gamma(x)$ where

$$
\left(\models_{\mathfrak{N}} \gamma(\sharp \psi)\right) \Leftrightarrow\left(\models_{\mathfrak{N}} \psi\right)
$$

for all sentences $\psi$, since : 
- Extending the language of $\models_{\mathfrak{N}}$ to include a binary function symbol $h$ and for every $\phi\left(x_{1}, \ldots, x_{n}\right)$ an n-ary function symbol $K_{\phi\left(x_{1}, \ldots, x_{n}\right)}\left(x_{1}, \ldots, x_{n}\right)(n>0)$, we will take $f$ to be a recursive bijection mapping the formulas of our new language onto the constants of our language.

- We will extend $\mathfrak{N}$ to $\mathfrak{N}^{*}$ so that:

* For each $\phi\left(x_{1}, \ldots, x_{n}\right)$ and $a_{1}, \ldots, a_{n} \in\left|\mathfrak{N}^{*}\right|=|\mathfrak{N}|=\mathbb{N}$ we have

$$
K_{\phi\left(x_{1}, \ldots, x_{n}\right)}^{\mathfrak{A}^{*}}\left(a_{1}, \ldots, a_{n}\right)=1 \Leftrightarrow \models_{\mathfrak{N}} \phi\left(a_{1}, \ldots, a_{n}\right)
$$

where we have slightly abused notation by substituting $a_{1}, \ldots, a_{n}$ into the formula rather than substituting the constants $c_{a_{i}}$ for $a_{i}$. We note that

$$
\left(\models_{\mathfrak{N}^{*}} K_{\phi\left(x_{1}, \ldots, x_{n}\right)}\left(a_{1}, \ldots, a_{n}\right)=1\right) \Leftrightarrow\left(\models_{\mathfrak{N}} \gamma\left(\sharp \phi\left(a_{1}, \ldots, a_{n}\right)\right)\right.
$$

* For each $\phi\left(x_{1}, \ldots, x_{n}\right)$ we will have

$$
h^{\mathfrak{N}^{*}}\left(f\left(\phi\left(x_{1}, \ldots, x_{n}\right)\right)^{\mathfrak{N}^{*}}, c^{\mathfrak{N}^{*}}\right)=f\left(\phi\left(x_{1}, \ldots, c\right)\right)^{\mathfrak{N}^{*}}
$$

where because $f$ is recursive we have a $\sigma_{2}(x, y, z)$ in the language of $\mathfrak{N}$ where

$$
\left(A_{E} \vdash \sigma_{2}\left(c_{1}, c_{2}, c_{3}\right)\right) \Leftrightarrow \models_{\mathfrak{N}^{*}} h\left(c_{1}, c_{2}\right)=c_{3}
$$

and again we note that

$$
\left(\models_{\mathfrak{N}^{*}} h\left(c_{1}, c_{2}\right)=c_{3}\right) \Leftrightarrow\left(\models_{\mathfrak{N}} \gamma\left(\sharp \sigma_{2}\left(c_{1}, c_{2}, c_{3}\right)\right)\right)
$$

- It follows from the above that we can give a recursive function $f^{*}$ mapping from and to the constant symbols of our language, where

$$
\left(\models_{\mathfrak{N}^{*}} \phi\right) \Leftrightarrow\left(\models_{\mathfrak{N}} \gamma\left(f^{*}(f(\phi))\right)\right)
$$

where because $f^{*}$ is recursive we then can give a formula $\sigma_{1}(x)$ in the language of $\mathfrak{N}$ where

$$
\left.\left(A_{E} \vdash \sigma_{1}(f(\phi))\right) \Leftrightarrow \models_{\mathfrak{A}^{*}} \gamma\left(f^{*}(f(\phi))\right)\right)
$$

- We take $g(x)=K_{\sigma_{1}}(x)$.

Noting that $\models_{\mathfrak{A}^{*}}$ is a model of our original language union $\{g, h\}$, we have met the requirements for $\models_{\mathfrak{A}^{*}}$ being an expressive resolution for $\models_{\mathfrak{A}}$ and consequently $\models_{\mathfrak{A}}$ is expressive which contradicts our Corollary 5.8.

II. Because partial Boolean algebras are atomic complete (all atomic formulas have truth value, see [7]), Theorem 5.7 gives that the expressive resolution for a semantic truth assignment when it exists, 
can not be a partial Boolean algebra. Thus no expressive partial Boolean algebra would have an expressive resolution that is a partial Boolean algebra. To be fair to partial Boolean algebras nothing we have presented rules out the existence of a codified semantic truth assignment giving a partial Boolean algebra and being expressible in itself. But when a partial Boolean algebra can be expressed in itself, we then have that the property of being expressed in itself either:

* Does not have the ability to define a substitution function $h$ for the encoding function $f$ witnessing the partial Boolean algebra being expressed in itself.

Or

* The partial Boolean algebra when extended by definition to capture $g$ and $h$ (where $g$ and $h$ are functions given by formulas which witnessing the fact that the partial Boolean algebra is expressed in itself, and given by the failure of the above ${ }^{*}$, respectively) gives a partial Boolean algebra which is not expressed in itself.

In either case the partial Boolean algebra presents limitations on its ability to meaningfully comment about itself, which we note are not limitations of Anti-Structures. The above limitation can be characterized as an inability to name functional processes as functions and extend the significance of the meaning of these functional process to account for it being named as a function. Naturally if the aforementioned limitation is not seen as a limitation of our universe, then the ability to capture the logic of our universe at its most fundamental level with a partial Boolean algebra is in question. Naturally if the antecedent of the last sentence is not held true then we would not necessarily have reason to worry about its consequence.

2. From the above it is clear that the notion of expressed captures when true things asserted by a mechanism for assigning truth can be encoded and captured by a formula under that mechanism (i.e. represented in the said mechanisms truth assignments). But more motivational and a less rigorous way to view the notion of expressed might be to think about a reasonable to do list one might write (indeed such a list tangibly encodes possible real world events and is even subject to a logical analysis), and then drawing the analogy that the list is a representation, we could call the list expressed if completed, and giving existential crisis if never completed. Taking our analogy a bit further under a clear suspension of rigor, letting $\Sigma$ be the assignment of truth given by what a physicist can observe in our universe, and then assuming that $\Sigma$ is a codified semantic truth assignment, we might then consider the following as examples of existential crisis

a. Again under a suspension of rigor. We might take there to be an ideal model of the Newtonian universe and a codified semantic truth assignment $\Sigma_{1}$ for it. Then also assuming that a physicist can use the axioms of Newtonian physics to calculate what is true and untrue 
about the Newtonian universe, we might further take it for granted that this gives a representation of the Newtonian universe in $\Sigma$. As say the orbit of Mercury contradicts the orbit it should have in the Newtonian universe, we would say that the Newtonian universe is represented in $\Sigma$ but not expressed in $\Sigma$ and therefore is in existential crisis in $\Sigma$.

b. Following the lack of rigor above. We might assume that the wave model for light which lead to the ultraviolet catastrophe (RayleighJeans catastrophe) of the early 20th century, had a codified semantic truth assignment $\Sigma_{2}$ which was represented in $\Sigma$ (from $a$ above), but not expressed in $\Sigma$ and therefore in existential crisis in $\Sigma$.

We note that Planks resolution to the ultraviolet catastrophe is considered as the foundation of quantum mechanics. It is well known that in Planks resolution the amplitude of the wave representation of light was restricted to integer multiples of the Plank constant where then no amplitude which was not an integer multiple of the Plank constant could occur. Phrasing things in terms of the language of our paper and again holding to a lack of rigor, we might say that the existential crisis of $\Sigma_{2}$ with regard to the amplitudes of of light was resolved with an Anti-Structure proxy $\models_{\mathfrak{A}}^{A}$ for $\Sigma_{2}$ where $\models_{\mathfrak{A}}^{A}$ is clearly very similar to the Anti-Structure given in Example 3.13. Of course the lack of rigor here makes this statement more of an opinion and should motivate questions to bring it out of the realm of opinion and determine the truth of the matter. We refer the reader to questions.... The orbit of Mercury from $a$ above was resolved by Einsteins general relativity and does not seem to directly speak to a potential Anti-Structure proxy. However we note that special relativity prevented a contradiction with Maxwell's equations and light, if one was to observe light in a vacuum while traveling at the same velocity. Here the contradiction being that a photon according to Maxwell's equations can only be observed as traveling at the speed of light and then the contradiction being avoided was that of the photons non-existence to an observer traveling at a velocity which would cause the observed velocity of the photon to be reduced. Here we simply note that there is a clear correlation between the impossibility of non-existence of a photon under the aforementioned observation conditions and an Anti-state in an Anti-Structure. Naturally this hypothetical connection should be more rigorously addressed with formal questions. To these ends we refer the reader to Questions 1 and 2 in Section 5.3. We note that in a recent paper by [9], entanglement was shown to be connected to the Plank length by the same assumption of no preference reference frame (NPRF) given by Einstein with regard to the photon.

3. In this comment we give some context for our choice of content for this paper as well as the motivation for the general nature of the following questions. In the summer of 2016 a version of this paper was submitted to the Annuals of Pure and Applied Logic. The earlier version of the 
paper called what is currently being named an Anti-state, a jump out. The content of the first version of the paper was mostly the theorems occurring before Theorem 3.25 of this paper and excluding Example 3.13. The 2016 submission modeled the double slit experiment by having:

- A sequence of starting points $s_{n}$ and a function $f$ acting on each $s_{n}$ where then $f^{m}\left(s_{n}\right)$ would represent $m$ steps of the transition of a particle emitted at moment $s_{n}$ from a source.

- Then taking certain terms $f^{m}\left(s_{n}\right)$ to be Anti-states (jump outs) caused the the set of $s_{i}$ 's where $s_{i}=x$ allowed for a formula $\phi$ having $f^{m}(x)$ as a terminal term to be defined, would give that $\phi$ only had a truth value for a specific subsequence of all of the $s_{i}$ 's.

- The probability of a $\phi$ being true was determined using the relative frequency measure for it being true on the sequence where $\phi$ was defined.

- In the paper it was noted that any real world data for the double slit experiment should be able to be matched using the aforementioned process.

The paper did not give any means for capturing interference and did not make any connection to the use of Planks constant and the restriction to integer values of continuous functions from $\mathbb{R}^{n}$ to $\mathbb{R}$ which we have made in this paper. The paper was not accepted for publication because the treatment of the jump out notion was to simple and it failed to give a full explanation for the quantum mechanics involved in the double slit experiment, although the notion of jump out was taken to be of interest. This was a fair assessment although the intent of the paper was to show how context change could be a consequence of a jump out (Anti-state). This paper is very much attempting to address the referees critiques of the last submission. When attempting to address the question of how a jump out(Anti-state) gives a meaningful understanding to Quantum Mechanics beyond capturing context change, we found that considerations of $a$ and $b$ of Comment 2 lead us to see the significance of the Anti-state may be in resolving our notion of existential crisis, and from Comment 1 that the need to resolve such issues might be fundamental to the expressive nature of our universe. In regard to the issues raised by Comments 1,2 we came to the conclusion that it would not suffice to show that an Anti-state is necessary in avoiding a contradictions of our physics but rather that the most fundamental questions at hand are:

- What is the expressive nature of our universe?

- How expressive is any given asserted model of our universe?

Our Theorems 3.25, 4.2 can be seen as establishing a general means for constructing Anti-Structure models in which problems of our physics may be expressed. The fact that the models given by Theorems 3.25 and 4.2 are governed entirely by their intentional construction, which seems to be 
at odds with Anti-states giving an explanation of anything in physics that one would call natural, leads very much to questions of what it means to have an expressive structure that does not make such an appeal to outside authority to suit its our purposes (A natural expressive structure). With regard to our aforementioned intentions we note that even if in regard to [9] paper we might have adapted our 2016 example to incorporate Plank lengths in determining where an $f^{m}\left(s_{i}\right)$ was a jump out, and then find some deeper connection between the example and double slit experiment, this should still not have been sufficient for explaining why the laws of our universe dictate these seemingly necessary unobservables, such as fractions of Plank lengths. In the questions that follow we do not endeavorer the lofty goals of addressing why our universe requires governing laws which require the aforementioned unobservables, but rather we ask questions on the lines of determining what types of rules govern expressive semantic truth assignments and Anti-Structures.

\subsection{Futher Questions}

1. To what degree can the following be formally given as an existential crisis:

a. The ultraviolet catastrophe

b. The non-existence of a photon if it had zero relative speed to an observer

2. To what degree can Anti-states be seen to be connected to resolutions to the problems presented by $a$ and $b$ from 1 above?

3 . To what degree should a proposed model of our universe be able to represent itself and verify true statements about itself?

4. Noting that an Anti-Structure $\models_{\mathfrak{A}}^{A}$ for a system $Y$ has the requirement: For all terms $t$ of the language, $t=t$ is true if and only if there exists a constant symbol $c$ where $(t=c) \in Y$.

We ask:

a. How strong is the above requirement?

b. How strong is the above requirement under the additional assumption that $t=t$ is always true?

c. Is there an expressive Anti-Structure which gives that the above requirement is true about itself?

5. Relatively speaking a relationship $R \subseteq X^{n}$ is definable in a theory $T$ when there exists an encoding of $X$ into the constant symbols of the language of $T$ and a formula $\phi_{R}$ where $\phi_{R}\left(c_{x_{1}}, \ldots, c_{x_{n}}\right) \in T$ if and only if $\left\langle x_{1}, \ldots, x_{n}\right\rangle \in R$. Noting that in a system $Y$ for an An-Structure, we can have:

* A relation $R$ and a formula $\phi_{R}$ where for all variable to constant assignments $c v, \phi_{R}[c v] \in Y$ and $\phi_{R}$ defines $R$. 
* A formula $\psi$ where for all variable to constant symbols $c v, \psi[c v] \in Y$.

* There exists a variable to constant assignment $b v$ where $\left(\phi_{R} \vee \psi\right)[b v] \notin$ $Y$

We ask:

a. What is the correct definition of a relation $R$ being definable in a system $Y$ for an Anti-Structure?

b. Given the above mentioned $\psi$ and $b v$ give a context change where $\phi_{R}$ has no truth value, is there a measure $\mu$ for definable relationships in a system $Y$ for an Anti-Structure, which gives the degree to which $\phi_{R}$ has truth value and correctly defines $R$ ?

c. Given $\mu$ from item $b$ can be defined for each system for an AntiStructure, is there an expressive Anti-Structure which can make statements about its measure $\mu$ for its definable relations?

We additionally note: For a $\kappa$ simple contextual model having measurement sets $m_{\alpha}$ for $\alpha<\kappa$, if for all $\alpha, S \subseteq m_{\alpha}$, and $\psi_{\alpha}$ is a tautology where $\operatorname{ter}(\psi)=m_{\alpha}$, then for any $\phi$ where $\operatorname{ter}(\phi)=S$ and a variable to constant assignment $c v$, we have that checking the truth of $\left(\psi_{\alpha} \wedge \phi\right)[c v]$ in the $\kappa$ simple contextual model, could be viewed as checking to see if $S[c v]$ is in state $\phi[c v]$ (or in the form $\phi[c v]$ ) under the measurement $m_{\alpha}[c v]$ (where we appeal to an intuitive notion of form or state here).

6. Taking definable in an Anti-Structure to mean definable in the system for the Anti-Structure, what characterizes an Anti-Structure $\models_{\mathfrak{A}}^{A}$ and a non-trivial class of Anti-Structures $S$, where every Anti-Structure in $S$ is definable in $\models_{\mathfrak{A}}^{A}$, while in turn $\models_{\mathfrak{A}}^{A}$ is definable in each Anti-Structure contained in $S$ ?

7. Following from the comment on how constructed our examples from Section 4 are, we ask if there can be a meaningful measure of how constructed an Anti-Structure model is?

8. If there is a measure of how constructed Anti-Structure models are, then can this measure be extended to arbitrary models for physics?

\section{References}

[1] Herbert B. Enderton, A mathematical Introduction to logic, 2nd ed., Harcourt/Academic Press, Burlington, MA, 2001. MR1801397

[2] Richard P. Feynman, Robert B. Leighton, and Matthew Sands, The Feynman lectures on physics. Vol. 2: Mainly electromagnetism and matter, Addison-Wesley Publishing Co., Inc., Reading, Mass.-London, 1964. MR0213078

[3] Kenneth Kunen, Set theory, Studies in Logic and the Foundations of Mathematics, vol. 102, North-Holland Publishing Co., Amsterdam, 1983. An Introduction to independence proofs; Reprint of the 1980 original. MR756630

[4] Simon Kochen and E. P. Specker, Logical structures arising in quantum theory, Theory of Models (Proc. 1963 Internat. Sympos. Berkeley), North-Holland, Amsterdam, 1965, pp. 177-189. MR0205582 
[5] _ The problem of hidden variables in quantum mechanics, J. Math. Mech. 17 (1967), 59-87, DOI 10.1512/iumj.1968.17.17004. MR0219280

[6] Simon Kochen, A reconstruction of quantum mechanics, Found. Phys. 45 (2015), no. 5, 557-590, DOI 10.1007/s10701-015-9886-5. MR3326202

[7] M. Dalla Chiara, R. Giuntini, and R. Greechie, Reasoning in quantum theory, Trends in Logic-Studia Logica Library, vol. 22, Kluwer Academic Publishers, Dordrecht, 2004. Sharp and unsharp quantum logics. MR2069854

[8] Garrett Birkhoff and John von Neumann, The logic of quantum mechanics, Ann. of Math. (2) 37 (1936), no. 4, 823-843, DOI 10.2307/1968621. MR1503312

[9] W. M. Stuckey, Michael Silberstein, Timothy McDevitt, and T. D. Le, Answering Mermin's challenge with conservation per no preferred reference frame, Scientific Reports 10 (2020), DOI 10.1038/s41598-020-72817-7.

[10] John von Neumann, Mathematical foundations of quantum mechanics, Princeton University Press, Princeton, NJ, 2018. New edition of [ MR0066944]; Translated from the German and with a preface by Robert T. Beyer; Edited and with a preface by Nicholas A. Wheeler. MR3791471 